\title{
Growth and PhysioChemical Properties of Second-Order Nonlinear Optical L-Threonine Single Crystals
}

\author{
G. Ramesh Kumar ${ }^{1}$ and S. Gokul Raj ${ }^{2}$ \\ ${ }^{1}$ Department of Physics, University College of Engineering Arni, Anna University Chennai, Arni 632317, India \\ ${ }^{2}$ Department of Physics, National Institute of Technology, Tiruchirappalli 620015, India
}

Correspondence should be addressed to G. Ramesh Kumar, rameshvandhai@yahoo.co.in

Received 16 March 2009; Revised 15 November 2009; Accepted 16 December 2009

Recommended by Pavel Lejcek

The present aim of the paper is to grow and to study the various properties of L-threonine amino acid single crystal in various aspects. Crystal growth of L-threonine single crystals has been carried out with the help of crystallization kinetics. pH and deuteration effects on the properties of the grown crystals have been studied and the results presented in a lucid manner. The various second-order NLO parameters were evaluated using anharmonic oscillator model. Particle and ion irradiation effects on structural, optical, and surface properties of the crystals have also been studied in detail.

Copyright (C) 2009 G. Ramesh Kumar and S. Gokul Raj. This is an open access article distributed under the Creative Commons Attribution License, which permits unrestricted use, distribution, and reproduction in any medium, provided the original work is properly cited.

\section{Introduction}

1.1. Introduction. One of the important applications in photonic technology is the use of nonlinear optical (NLO) effects. A nonlinear optical effect is the interaction of an electromagnetic field of high intensity laser light with a material. Materials with large nonlinear optical susceptibilities are of importance in optoelectronics, where they are used in the construction of the optical analogues of the circuit elements-modulators, amplifiers, rectifiers, switches, and so forth, of conventional electronics. Some organic materials have exceptionally large nonlinear susceptibilities, the origins of which can be tracked back to the molecular electronic properties and in the recent years, a great deal of effort has been expanded on their identification and development [13 ]. The material may be in number of different macroscopic forms: crystals, polymers, thin films, and so forth, but always with the proviso that for second-order effects, the structure must at least on average be noncentrosymmetric. Second harmonic generation ( $\mathrm{SHG}$ ) is one of the techniques used to investigate the second-order nonlinearities in new materials and if the SHG tensor, linear optical properties, and the details of the crystal structure with molecular coordinates can be established, it is possible to make reasonably accurate predictions of other second-order parameters such as the linear electro-optic coefficients.

The linear and nonlinear optical properties of organic crystals are highly anisotropic, and the behaviour of the nonlinear output is affected critically by the values of the linear dielectric tensors at the fundamental and second harmonic frequencies. SHG and the linear electro-optic effect both have their origins in the second-order electric susceptibility as can be seen from the following simplified analysis, where the vector and tensor indices are temporarily suppressed.

The time dependent part of the electric field of plane polarized light is given by

$$
E=E_{\omega} \sin \omega t
$$

and that the light passes through a crystal across which a static field $E_{0}$ is applied in the same direction as the radiation electric field. Then to second order, the polarization of the crystal is

$$
\begin{gathered}
P=\varepsilon_{0}\left[\chi^{(1)}\left(E_{0}+E_{\omega} \sin \omega t\right)+\chi^{(2)}\left(E_{0}+E_{\omega} \sin \omega t\right)^{2}\right], \\
P=P^{(0)}+P^{(1)}+P^{(2)},
\end{gathered}
$$


where

$$
\begin{aligned}
& P^{(0)}=\varepsilon_{0}\left(\chi^{(1)} E_{0}+\chi^{(2)} E_{0}^{2}+\frac{1}{2} \chi^{(2)} E_{\omega}^{2}\right), \\
& P^{(1)}=\varepsilon_{0}\left(\chi^{(1)}+2 \chi^{(2)} E_{0}\right) E_{\omega} \sin \omega t, \\
& P^{(2)}=-\frac{1}{2} \varepsilon_{0} \chi^{(2)} E_{\omega}^{2} \cos 2 \omega t .
\end{aligned}
$$

The linear polarization can be written as

$$
P^{(1)}=\varepsilon_{0} \chi_{\mathrm{eff}} E_{\omega} \sin \omega t,
$$

where $\chi_{\text {eff }}=\chi^{(1)}+2 \chi^{(2)} E_{0}$ is an effective susceptibilities depending linearly on the static field leading to an effective refractive index " $n$ "

$$
n=\left(1+\chi_{\mathrm{eff}}\right)^{1 / 2} \simeq n_{\omega}+\left(\frac{\chi^{(2)}}{n_{\omega}}\right) E_{0},
$$

where $n_{\omega}$ is the refractive index for light of frequency $\omega$ in the absence of second field. The lowest-order correction term varies with the first power of the low frequency field. This is termed as linear electro-optic Pockel's effect, often measured in bulk materials through electric field induced birefringence.

The polarization $P^{(2)}$ is at a frequency $2 \omega$ and leads to second harmonic generation. Both SHG and the Pockels effect originate from $\chi^{(2)}$, although the frequency dependence of the nonlinear susceptibility must be taken into account when attempting to relate the two effects. Detailed accounts of the second order nonlinear optical effects have been discussed by Yariv (1975) [4].

A general feature of second order NLO susceptibilities is that they can be nonzero only in noncentrosymmetric materials. For, if the direction of the field is reversed, then in a centrosymmetric material the induced polarization must also reverse, but the second order terms containing the product of two field components do not change sign.

1.2. Molecular Origin of $\chi^{(2)}$. Organic crystals are formed through the action of nonvalence intermolecular forces. As a consequence, the individual molecule retain their identities to a much greater extent than in the case for the atomic or ionic groups semiconductors or inorganic insulators. Macroscopic crystalline susceptibilities can therefore be related to molecular polarizabilities and hyperpolarizabilities, although internal field corrections must be applied in making a quantitative connection between the microscopic and macroscopic quantities.

The electric field of the radiation induces an electric dipole in the molecule

$$
\delta_{\mu i}=\alpha_{i j} E_{j}+\beta_{i j k} E_{j} E_{k}+\cdots,
$$

where for the first hyperpolarizability $\beta$ to be nonzero, the molecule itself must be noncentrosymmetric. High values of $\chi^{(2)}$ are derived from high values of $\beta$ and the exceptionally large values found in certain organic molecules can be traced to the electronic hyperpolarizabilities of these molecules.
In trying to identify the molecules with this property, the following three general features should be considered.

(i) The electronic structure should be effectively coupled to the electric field of radiation.

(ii) Resonance or preresonance enhancement should be used to magnify the response.

(iii) The molecule should be effectively noncentrosymmetric.

In standard time-dependent perturbation theory, the perturbed molecular ground state is expanded as a sum over of all the electronic states of the unperturbed molecule. Usually, for the molecules of interest, the large value of $\beta$ is accounted by the contribution of one particular virtual excited state.

Assuming that the effect is essentially one dimensional, the following formula can be derived (Bloembergen and Shen) [5]:

$$
\beta_{x x x}=\frac{\left(3 e^{2} / 2\right) \hbar^{2}\left[|\langle g|x| n\rangle|^{2} \Delta \mu_{n g} \omega_{n g}^{2}\right]}{\left[\omega_{n g}^{2}-2 \omega^{2}\right]}\left(\omega_{n g}^{2}-\omega^{2}\right) .
$$

The formula which has provided a great deal of guidance in the selection of the types of molecules to be investigated with the aim of finding exceptionally large first hyperpolarizabilities can be interpreted as follows.

The states $\langle g|$ and $|n\rangle$ are, respectively, the electronic ground state and the main contributing electronic excited state; the quantity $h \omega_{n g}$ is the excitation energy from $\langle g|$ to $|n\rangle$; " $\omega$ " is the angular frequency of the input radiation. The square modulus of the transition moment $\langle g|x| n\rangle$ is proportional to the oscillator strength of the transition and measures the coupling of the radiation field to this transition. $\Delta \mu_{n g}$ are the change in dipole moment $\left(\mu_{n}-\mu_{g}\right)$ on excitation and is the quantity that incorporates the essential effect of the lack of a centre of symmetry in the part of the electronic system involved in the $\langle g|$ to $|n\rangle$ transition. The nonzero values of $\Delta \mu_{n g}$ are associated with an asymmetric change in the electronic distribution. The values of $\beta$ can be greatly enhanced by working under conditions, where the factor $\left[\omega_{n g}^{2}-2 \omega^{2}\right]$ is small-an effect described as preresonant enhancement.

1.3. Second-Order Nonlinearity in Hydrogen-Bonded Amino Acids. Amino acids are interesting materials for second order NLO applications as they contain a proton donor carboxylic acid $(-\mathrm{COO})$ group and the proton acceptor amino $\left(-\mathrm{NH}_{2}\right)$ group in them. Crystalline salts of amino acids and their derivatives are one of the direction for searching new second order NLO materials. The much attracted salts of L-arginine [6-9], L-histidine [10-13], and L-Lysine [14-16] complexes have been reported earlier. The enantiomorphic " $D$ " and "L" residues of amino acids always crystallize in noncentrosymmetric space groups. The optical activity of amino acids favours for the generation of second harmonics and their molecular structures are stabilized through hydrogenbonded networks of either by $\mathrm{O}-\mathrm{H} \cdots \mathrm{O}$ or $\mathrm{N}-\mathrm{H} \cdots \mathrm{O}$ or both bonds. The dipole-dipole orientations of $\mathrm{H}$-bonded 
chiral molecules form pairs in parallel fashion which gives a nonzero first-order hyperpolarizability. The influence of strong and very strong hydrogen bonds on the nonlinear optical properties of these types of crystals was also considered earlier [17]. However the role of hydrogen bonding on the NLO properties of crystals is still not very well known. Besides, amino acids are more flexible to structural changes with good thermal strength and wide transparency in the UV-Vis-NIR range. Hence, a new strategy has been developed to increase the first-order hyperpolarizabilities based on acid-base interactions. The increase in second order NLO coefficients and their improvements in mechanical and thermal strength were observed by many investigators [18-20].

1.4. Crystal Growth. The field of crystal growth is interdisciplinary, as it needs expertise in fields such as chemistry, physics, mathematics, and crystallography. Growths of crystals essentially involve heat, and mass transfer phenomena as well as convection effects. The related temperature and concentration fields have a dominant effect on the quality of the growing crystals. Therefore appropriate control of fluid dynamics and a good understanding of the convection phenomena are very important for the growth of high quality crystals. Convection is the transport of momentum, heat, and mass due to the motion of a fluid. The role of fluid flow and convection in crystal growth has already been studied extensively [21].

1.4.1. Growth from Solutions. Crystal growth from solution is based on the existence of a metastable region, where formation of crystalline nuclei in the solution volume is impossible, but it is possible to grow crystals on a seed. Figure 1 presents a part of a classical phase diagram of a twocomponent system for a solute with a positive temperature coefficient of solubility $d C_{0} / d T>0[22]$.

The diagram can be described in terms of three zones.

(i) The stable zone of unsaturated solution where crystal growth is impossible.

(ii) The metastable zone where spontaneous crystallization is improbable but crystal growth on a seed occurs.

(iii) The labile zone of spontaneous nucleation.

Cooling a solution with concentration " $C$ " from the initial point $A^{I}$ leads to the $A$ on the solubility curve. At this point, the solution becomes saturated and can exist in equilibrium with the solid if $C=C_{0}\left(T_{0}\right)$. Further cooling leads to a state where the concentration $C_{0}(T)$ at a given temperature and the solution becomes supersaturated. However, spontaneous formation of the solid phase does not occur in this region until a point $B$ on the metastable boundary is reached. If crystallization in a solution saturated at the temperature $T_{0}$ starts at the temperature $T$, the position of the metastable boundary can be expressed in the units of maximum overcooling $\Delta T_{\max }=T_{0}-T$, the absolute supersaturation

$$
\Delta C_{\max }=C_{0}\left(T_{0}\right)-C_{0}(T),
$$

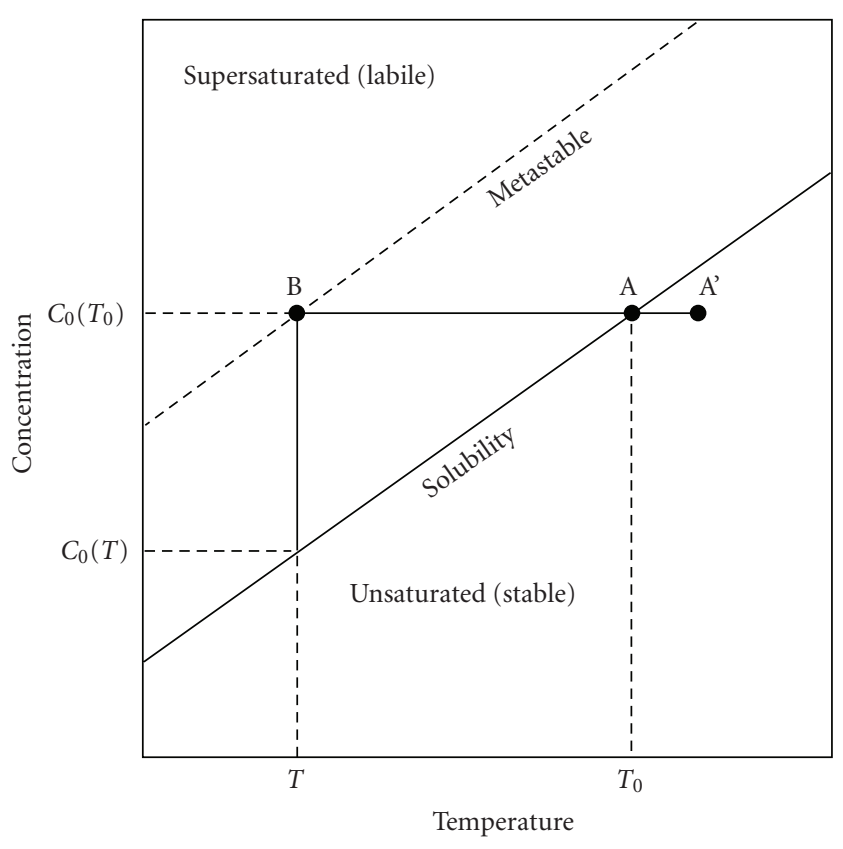

FIGURE 1: Phase diagram for a binary system of solid-liquid.

or in the units of relative supersaturation

$$
S=\frac{\Delta C_{\max }}{C_{0}\left(T_{0}\right)} .
$$

One more important parameter that characterizes the metastable zonewidth is the induction period $\tau_{i}$. It corresponds to the time during which the system can remain without spontaneous nucleation at constant supersaturation within the metastable zone.

Growing a crystal without spontaneous nucleation is possible only within the metastable zone. In homogeneous nucleation, the formation of a particle of a new phase leads to the change in Gibbs free energy $\Delta G$, determines the two process

(i) joining molecules into cluster,

(ii) the formation of the surface area of the new particle.

The change in Gibbs free energy depends on change in the chemical potential during the formation of the cluster of radius " $r$ ". Maximum value of $\Delta \mathrm{G}$ obtained from the condition $d(\Delta G) / d r=0$ corresponds to critical radius $r^{*}$ and hence the expression for critical free energy can also be obtained. The quantitative estimation of parameters like width of the metastable zone, degree of supersaturation, induction time, critical radius, and critical free energy decide the stability of the solution for the successful growth of crystals.

1.5. A Relay Mechanism of Crystal Growth in Amino Acids. Amino acids and their derivatives decompose before melting and do not have a precise melting point and hence high temperature solution growth techniques are not feasible for growing these types of crystals. Low-temperature solution 


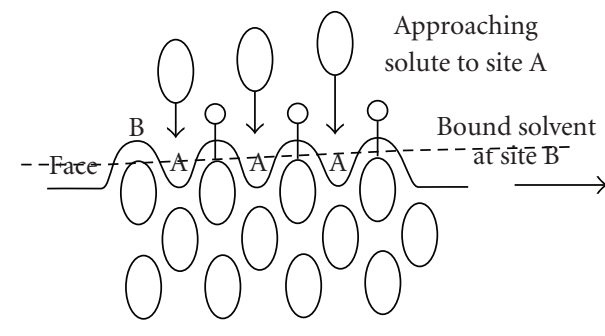

(a)

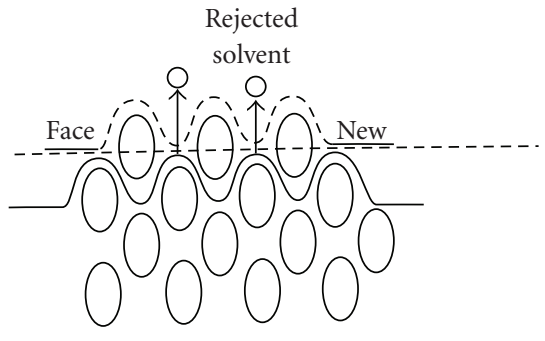

(b)

FIGURE 2: Mechanism of crystal growth by selective solvent binding in amino acids.

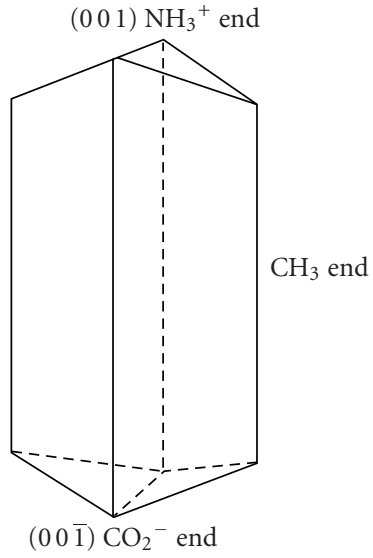

FIGURE 3: Packing arrangement of L-threonine as viewed down the $b$-axis.

growth is the best and well-suited method for the single crystal growth of amino acid crystals. A model for predicting the shape of amino acid crystals, crystallized from solution has also been predicted earlier [23]. Weissbuch et al. [24] have given a sterochemical approach for solvent-solute binding on the growth of polar amino acid crystals especially for amino acids having one carboxylate $\left(\mathrm{CO}_{2}{ }^{-}\right)$and one amino $\left(\mathrm{NH}_{3}{ }^{+}\right)$group like glycine, L-alanine, L-threonine, and so forth.

An entirely different approach involved through selective adsorption of solvent at a subset of molecular surface sites, of say type B and repulsion of solvent at the remaining set of surface sites, of say type A, on the crystal face. This is shown in Figure 2 (a), where the difference between the two types of sites was emphasized by assuming a corrugated surface such that the A-type site is a cavity and the B-type site is on the outside upper surface of the cavity. Figure 2(a) shows the B-type sites blocked by the solvent " $\mathrm{S}$ " and A-type sites unsolvated. Thus solute molecuales can easily fit into A-type sites. However once locked into positions (Figure 2(b)), the roles of the A- and B-type sites are essentially reversed and the solvent molecules which originally were bound to B-type sites would be repelled since they now occupy A-type sites. This cyclic process can lead to fast growth by a kind or "relay mechanism."

In such a situation, where desolvation is rate limiting, the free energy of incorporation of a solute molecule helps

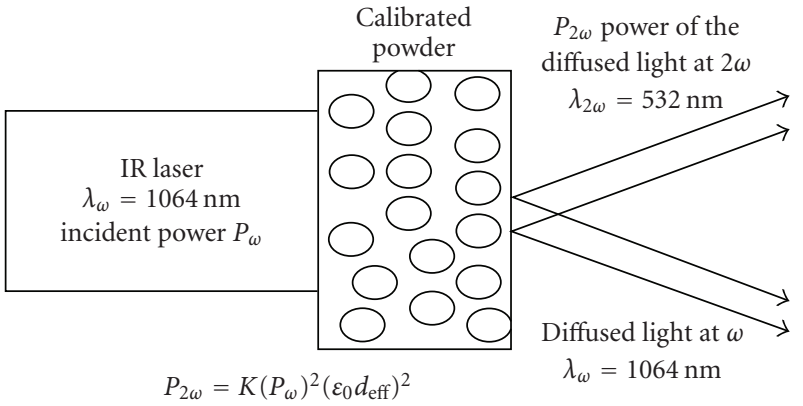

FIgURe 4: Principle of Kurtz-Perry powder SHG test.

to displace the bound solvent. This relay mechanism may de demonstrated for the crystals having polar axis. Crystals like L-alanine and L-threonine having similar packing features crystallize in the same space group $\mathrm{P} 2{ }_{1} 2_{1} 2_{1}$ of an orthorhombic system. Hence discussion can be confirmed to both of them. The zwitterionic molecules are aligned in such a way that $\mathrm{CO}_{2}{ }^{-}$groups at one end of the polar $c$-axis and $\mathrm{NH}_{3}{ }^{+}$groups at the opposite end. The crystal has a polar morphology, with the $\mathrm{CO}_{2}{ }^{-}$groups emerging at the $-\mathrm{C}$ end as shown in Figure 3.

The $\left(\begin{array}{ll}0 & 0\end{array}-1\right)$ face at the carboxylate end contains cavities. This face grows and dissolves faster in aqueous solution than the smoother amino face. Faster growth at the $\left(\begin{array}{lll}0 & 0 & -1\end{array}\right)$ side of the crystal in aqueous solution is due to the relay mechanisms of growth accordingly to the following arguments. The water molecules may be strongly bound by hydrogen bonds to the outer most layer $\left(\begin{array}{lll}0 & 0 & -1\end{array}\right)$ of $\mathrm{CO}_{2}{ }^{-}$ groups. In contrast, the pocket acts as a proton acceptor for the $\mathrm{NH}_{3}{ }^{+}$groups of the solute molecules. Replacement of the solute $\mathrm{NH}_{3}{ }^{+}$moiety by solvent water within the pockets yields repulsive or at best, weakly attractive interactions. The pockets will therefore be weakly hydrated and so relatively easily accessible to the approaching solute molecules. Higher crystal attachment energy also accounts for the faster growth of the crystals along the $c$-axis.

1.6. Kurtz-Perry Powder SHG Measurement. The second harmonic generation (SHG) efficiencies of the crystals under investigation have been evaluated using the conventional Kurtz-Perry powder technique [25] following the principle exhibited in Figure 4. 


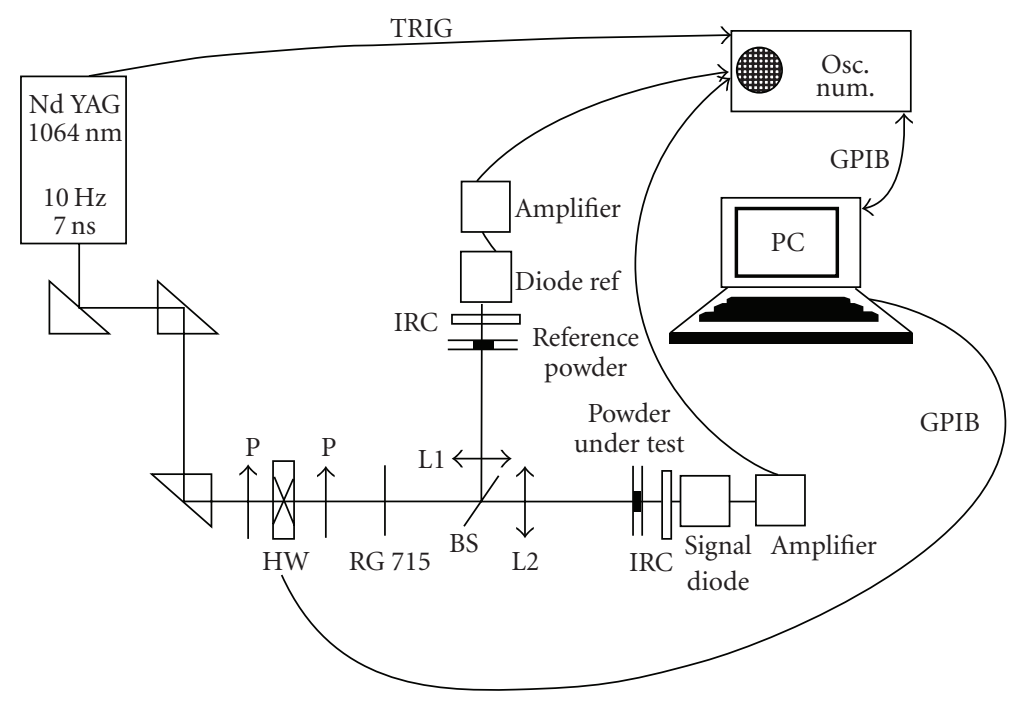

FIGURE 5: Kurtz-Perry powder SHG experimental setup.

Calibrated polycrystalline samples were illuminated by nanosecond pulses at the fundamental wavelength $\lambda_{\omega}=$ $1064 \mathrm{~nm}$, delivered by an Nd : YAG laser with a pulse width of 10 nanoseconds and a repetition rate of $10 \mathrm{~Hz}$. In the case of phase matchable crystals, with the powder SHG efficiency being an increasing function of the particle size, a large calibration (150-200 $\mu \mathrm{m}$ ) was chosen for both the powder under test and the phase matchable material taken as a reference sample (KDP).

Following the experimental setup represented in Figure 5, the fundamental beam is divided into two identical beams by a beam splitter BS. One of them illuminates the sample under study and the other one illuminates a reference sample. The two samples are powders of the same calibration placed in identical holders. The second harmonic powder diffused by the sample under test $\left(P_{2 \omega}\right)$ is measured as a function of second harmonic power diffused by the reference sample $P_{r 2 \omega}$ when the pump beam power is increased by rotating a half-wave plate ( $\mathrm{HW}$ ) between two parallel polarisers $P$.

The effective second harmonic coefficient of the sample could be found out using second harmonic beam powers $P_{2 \omega}$ and $P_{r 2 \omega}$. The output powers diffused by the sample under test and the reference are proportional to the square of their effective coefficients $d_{\text {eff }}$ and $d_{\text {reff }}$, the square of the incident beam power $P_{\omega}$ of the fundamental wave and also to a factor $K$ which depends on the refractive indices of the sample, but it may be considered approximately as a constant in this evaluation test.

This can be written as

$$
\begin{gathered}
P_{2 \omega}=K\left(\mathrm{~d}_{\mathrm{eff}}\right)^{2}\left\{P_{\omega}\right\}^{2}, \\
P_{r 2 \omega}=K\left(\mathrm{~d}_{\text {reff }}\right)^{2}\left\{P_{\omega}\right\}^{2} .
\end{gathered}
$$

Since SHG coefficient of the reference sample is known, $d_{\text {eff }}$ of the sample under test could be found out. It can be pointed out that this evaluation does not take into account the absorption of the samples in the visible part of the

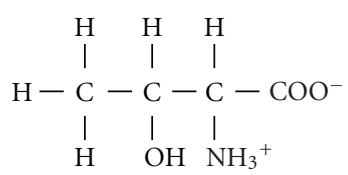

Figure 6: Zwitterionic state of L-threonine.

spectrum and therefore some of the effective coefficient may be underestimated.

1.7. L-Threonine. L-threonine is an uncharged neutral amino acid and a useful compound for its nonlinear optical properties. It contains two asymmetric carbon atoms with a single carboxylate $\left(\mathrm{CO}_{2}{ }^{-}\right)$and amino $\left(\mathrm{NH}_{3}^{+}\right)$group. The scheme of L-threonine molecule in the zwitterionic state is shown in Figure 6. Its crystal structure was first solved by Shoemaker et al. [26].

It crystallizes in noncentrosymmetric $\mathrm{P} 2{ }_{1} 2_{1} 2_{1}$ space group with 4 zwitterionic molecules in the unit cell linked by a 3-dimensional network of hydrogen bonds of unequal strength [27]. Its phase matching properties have already been reported with a very few details on the growth and crystal quality. Despite the phase matching loci of Lthreonine are similar to a uniaxial negative crystal like KDP, the material belongs to a negative biaxial one showing both type I and type II phase matchings. The third-order nonlinearity of L-threonine has been investigated through the Z-scan technique and the nonlinear index of refraction $\left(n_{2}\right)$ was found to be of the order $10^{-20} \mathrm{~m}^{2} / \mathrm{W}$ at $775 \mathrm{~nm}$ [28].

\section{Experimental Procedures on L-Threonine Single Crystals}

2.1. Crystallization Kinetics. Crystallization plays an important role in pure and applied science. Our ability to design crystals with desired properties and control over crystallization based on structural understanding are still limited. In 


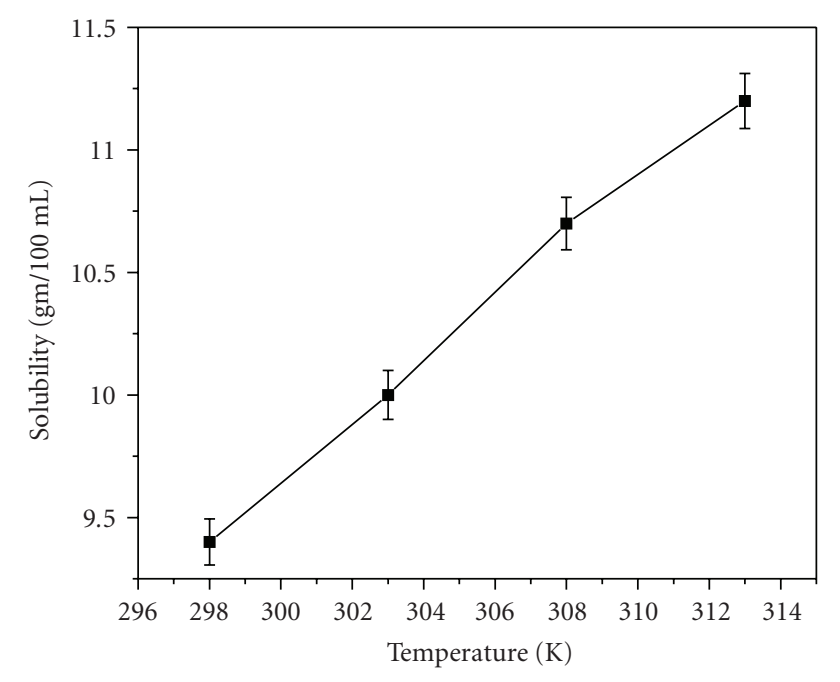

Figure 7: Solubility of L-threonine in water solvent.

particular, control of nucleation in polar crystals like amino acids, peptides, and sugar is significant in the sense that they have different growth rates at the opposite poles of a polar axis. Hence, it is necessary to investigate the nucleation thermodynamical nucleation parameters associated with the crystal growth process. The crystallization parameters could be found out experimentally so as to control nucleation.

2.1.1. Solubility and Solvent Selection. The crystal morphology of polar crystals is highly influenced by solvent selection. The role played by solvent-solute interaction in L-alanine, L-aspartic acid, L-asparagine was reported recently [29]. Tanaka and Matsuoka [30] proposed two properties that would influence the crystal habit, they are (i) the differences in both the heat of crystallization and (ii) in the dipole moments between the crystallization component and the solvent.

When a solvent having smaller dipole moment and the ideal solubility is selected, large three-dimensional crystals can be expected to grow. Based on the above views, the solubility of L-threonine has been tested in various solvents like water, methanol, ethanol, and their mixtures. Since Lthreonine is a polar amino acid and its dipole moment is nearly equal to that of water, deionized water was found to be the best solvent for the crystallization of L-threonine single crystals. The solubility diagram of L-threonine in water is given in Figure 7.

2.1.2. Interfacial Energy. The surface energy $(\sigma)$ between a solid crystal and the surrounding saturated solution is a crucial parameter to determine the rate of nucleation and growth of the crystal. Nucleation experiments have been employed to determine the value of surface energy of saturated aqueous solution by Nielsen and Söhnel [31]. From the experimental solubility data, the linear relationship between the surface energy of the solution and the molar concentration of the solute has been discussed by many authors [32, 33].

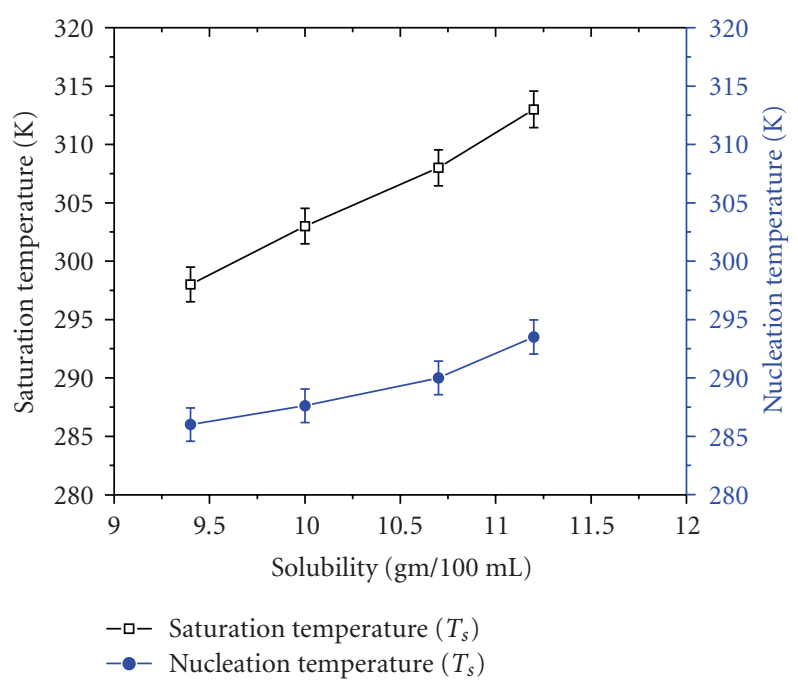

FIGURE 8: Metastable zonewidth at various saturation temperatures.

Bennema and Söhnel [34, 35] have derived the expression for the linear dependence of " $\sigma$ " with solubility from

$$
\sigma=\left(\frac{k T}{a^{2}}\right)\left[0.173-0.248 \ln \left(\chi_{m}\right)\right]
$$

where $\chi_{m}$ is the mole fraction of the solute, $T$ is the temperature in Kelvin, " $a$ " is the interionic distance, and $k$ is the Boltzmann's constant.

The above expression has been used to determine the surface energies at different temperatures using the solubility data. The calculated interfacial energies are in the range $0.76 \times 10^{-5}-1.09 \times 10^{-5} \mathrm{~J} / \mathrm{m}^{2}$. Since interfacial energy $(\sigma)$ is inversely proportional to growth rate $(R)$ of a crystal, the lesser $\sigma$ values obtained for L-threonine in aqueous solution are responsible for faster growth along the direction of polar axis of the L-threonine crystal.

2.1.3. Metastable Zone Width and Induction Period Measurements. Metastable zonewidth or Ostwald-Meirs region is defined as the degree of supercooling $(\Delta T)$ needed for one nucleus to be formed per unit volume in one second. It can be measured for various saturation temperatures using the conventional polythermal method [36, 37]. In this method, the saturated solution is cooled from the overheated temperature until the first visible crystal is obtained. Since the time taken for the formation of the first visible crystal after the attainment of the crystal nucleus is very small, the first crystal observed may be taken as the critical nucleus. Metastable zonewidth for various saturation temperatures has been shown in Figure 8. Induction periods of L-threonine aqueous solution were also found out by using the isothermal method [37].

The saturated solution was cooled to the desired temperature and was maintained at that temperature. The time taken for the formation of the first tiny crystal was measured and termed as induction period $(\tau)$ of the solution. Repeated trials were done to ensure the correctness of the observed values. 
TABLE 1: Critical radius $\left(r^{*}\right)$, critical free energy $\left(\Delta G^{*}\right)$, volume free energy $\left(\Delta G_{v}\right)$ calculated at various supersaturation values.

\begin{tabular}{|c|c|c|c|c|c|}
\hline S. no & Temp (in K) & $S=C / C^{*}$ & $\left(r^{*}\right) \times 10^{-6} \mathrm{~m}$ & $\left(\Delta G_{v}\right)$ in $\mathrm{Jm}^{-3}$ & $\left(\Delta G^{*} \times 10^{-16}\right)$ in $\mathrm{Jm}^{-2}$ \\
\hline \multirow{5}{*}{ (1) } & \multirow{5}{*}{298} & 1.01 & 10.14 & -1.52 & 33.24 \\
\hline & & 1.02 & 5.08 & -3.03 & 8.35 \\
\hline & & 1.03 & 3.41 & -4.52 & 3.76 \\
\hline & & 1.04 & 2.57 & -6.01 & 2.14 \\
\hline & & 1.05 & 2.06 & -7.46 & 1.38 \\
\hline \multirow{5}{*}{ (2) } & \multirow{5}{*}{303} & 1.01 & 10.25 & -1.49 & 33.87 \\
\hline & & 1.02 & 5.15 & -2.98 & 8.57 \\
\hline & & 1.03 & 3.45 & -4.45 & 3.83 \\
\hline & & 1.04 & 2.60 & -5.90 & 2.18 \\
\hline & & 1.05 & 2.09 & -7.34 & 1.41 \\
\hline \multirow{5}{*}{ (3) } & \multirow{5}{*}{308} & 1.01 & 14.86 & -1.47 & 101.45 \\
\hline & & 1.02 & 7.47 & -2.93 & 25.66 \\
\hline & & 1.03 & 4.99 & -4.38 & 11.47 \\
\hline & & 1.04 & 3.77 & -5.81 & 6.53 \\
\hline & & 1.05 & 3.03 & -7.23 & 4.22 \\
\hline \multirow{5}{*}{ (4) } & \multirow{5}{*}{313} & 1.01 & 10.55 & -1.44 & 35.65 \\
\hline & & 1.02 & 5.28 & -2.89 & 8.93 \\
\hline & & 1.03 & 3.53 & -4.32 & 4.00 \\
\hline & & 1.04 & 2.67 & -5.72 & 2.28 \\
\hline & & 1.05 & 2.14 & -7.12 & 1.47 \\
\hline
\end{tabular}

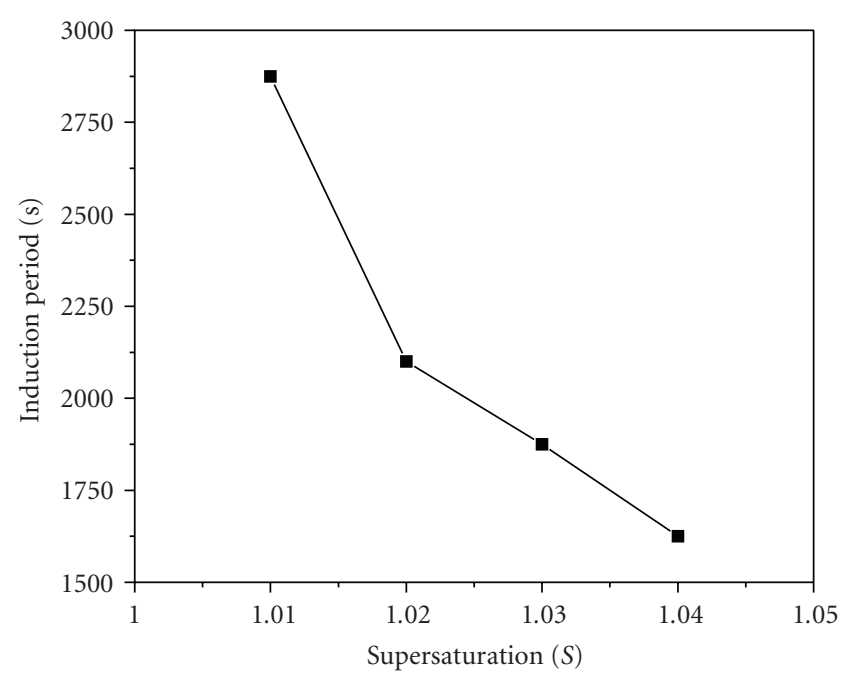

FIGURE 9: Induction period versus supersaturation for L-threonine.

2.1.4. Nucleation Kinetics. From the classical theory of nucleation for the case of crystal growth from aqueous solution, the supersaturation of a solution for a given temperature is

$$
S=1+\left(\frac{\Delta H_{s} \cdot \Delta T}{R T^{2}}\right)
$$

where $H_{s}$ is the heat of the solution. $\Delta T$ is the degree of supercooling and $R$ is the gas constant. $S$ can be calculated from the linear relation between $\ln \left(\chi_{m}\right)$ and $(1 / T)$.
Based on the linear relationship between $\ln \left(\chi_{m}\right)$ and $(1 / T)$, the value of $\Delta H / R$ was estimated and the supersaturation of the solution has been found out for different degree of super cooling $(\Delta T)$.

The driving force for the nucleation from the saturated solution is given by

$$
\Delta G_{v}=-\left(\frac{k T}{V}\right) \ln S,
$$

where $\Delta G_{v}$ is volume free energy change which usually possess negative values for liquid to solid phase transformation. $V$ is the specific volume of the solute molecule and $k$ is the Boltzmann constant.

The Gibb's free energy change involves in the formation of an L-threonine nuclei (spherical) which contains two terms, namely, the volume free energy term and the surface free energy term and it can be written as

$$
\Delta G=4 \pi r^{2} \sigma+\frac{4}{3} \pi r^{3} \Delta G_{v} .
$$

The critical radius and critical free energy barrier for nucleation are related by

$$
\begin{gathered}
r^{*}=-\frac{2 \sigma}{\Delta G_{v}}, \\
\Delta G^{*}=\frac{16 \pi \sigma^{3}}{3 \Delta G_{v}^{2}} .
\end{gathered}
$$

2.1.5. Crystallization Kinetics. From the above formulae, critical radius $\left(r^{*}\right)$, critical free energy $\left(\Delta G^{*}\right)$, volume free 


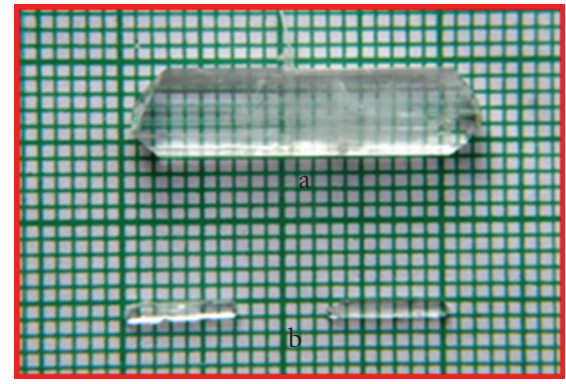

(a)

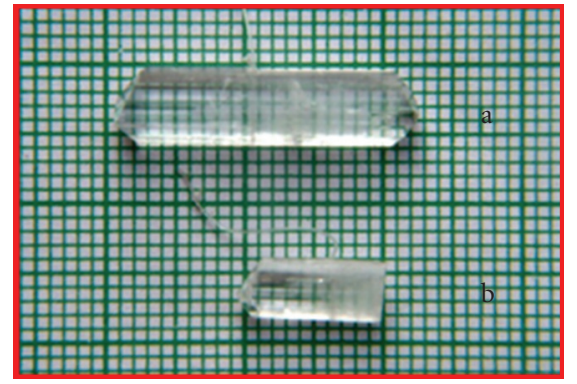

(b)

FiguRE 10: Photograph of L-threonine crystal grown from aqueous solution by slow cooling method.

energy $\left(\Delta G_{v}\right)$, metastable zonewidth, induction period, and interfacial energy values have been found out for various temperatures and are tabulated in Table 1.

Figure 9 represents the variation of induction period $(\tau)$ with supersaturation. From the curve, it is obvious that as supersaturation increases, the induction period value decreases and hence the nucleation is more probable for the higher value of supersaturation. The calculated interfacial energies for various temperatures were found to be very low when compared to other organic and inorganic solutions $[38,39]$. So growth rate of L-threonine single crystals would be higher than that of many NLO crystals. At all temperatures, zonewidth of the solution is considerably larger and it increases with an increase in temperature. The larger metastable zone widths at all temperatures greatly avoid the secondary nucleation formed during crystal growth. So, bulk crystals can be effectively grown from slow cooling technique at higher saturation temperatures.

Based on the ideas obtained from results of the graphs, the following growth conditions have been adopted for the bulk growth of L-threonine single crystals.

(i) As the multinucleation is more probable in higher supersaturation, crystals have been grown at low supersaturation values like 1.02, 1.03, and so forth, for all saturation temperatures.

(ii) Interfacial energy of the aqueous solution of Lthreonine was found to be low and the medium has less viscosity, hence water was used as a solvent for the growth process.

(iii) Zonewidth of the solution increases with saturation temperature and the growth of crystals at higher saturation temperatures like 60 and $70^{\circ} \mathrm{C}$ could greatly avoid secondary nucleation for a higher degree of supercooling. However the evaporation rate of the solvent used here is high at the higher saturation temperatures and hence the mother solution was saturated between 40 and $50^{\circ} \mathrm{C}$ for an uninterrupted growth of the crystals.

So, study of interfacial energy, induction period, supersaturation, and metastable zonewidth gives an idea to have controlled and uninterrupted growth throughout the growth period.
2.2. Crystal Growth of L-Threonine. In the present investigation, large and highly optical quality single crystals of L-threonine (2-Amino-3-hydroxybutyric acid) have been grown from aqueous solution by temperature lowering method. The grown crystals were subjected to different characterization studies such as single crystal XRD, FTIR, FT-Raman, and UV-Vis-NIR analyses. Dielectric, thermomechanical analyses and microhardness measurements were also carried out for L-threonine single crystal. Thermal stability of the grown crystal has been studied by DSC and TGA-DTA analyses. Kurtz-Perry SHG test was also carried out to confirm the second order nonlinear optical property of the grown crystal.

High purity L-threonine (99.9\%) was used for the crystal growth experiments. The solubility of L-threonine was found to be very high in water compared to pure organic and mixed solvents and hence deionised water was used as a solvent. Small transparent single crystals, free of macro defects, obtained by spontaneous nucleation from the saturated aqueous solution of L-threonine were selected as seed crystals. Mother solution of L-threonine was saturated at $40^{\circ} \mathrm{C}$ and a seed was introduced into the saturated solution placed in a constant temperature bath (accuracy $\pm 0.01^{\circ} \mathrm{C}$ ) at $40^{\circ} \mathrm{C}$ and the solution was maintained at this temperature for 24 hours. The solution was then allowed to cool at a rate of $0.1^{\circ} \mathrm{C} /$ day under constant stirring. Transparent crystals with defined morphology elongated along $c$-axis were grown after a typical growth period of 30 days; that were shown in Figure 10.

2.2.1. Crystal Morphology. The morphology of the grown crystal was measured using an Enraf-Nonius CAD4 singlecrystal diffractometer. The morphological planes are made up of $\{110\},\{120\}$, and $\{011\}$ faces. The length of the crystal is elongated along the $c$ direction (shortest unit cell axis). The top and the bottom planes of the crystal are Friedel pairs. It is worthwhile to note here that the morphology of L-threonine is quite similar to that of L-alanine single crystals [40] and is shown in Figure 11.

2.3. Influence of $p H$ on the Growth of L-Threonine Single Crystals. It is well known that quality and the physical properties of crystals grown from solution are considerably influenced by $\mathrm{pH}$ of the solution. This has been investigated in a number 


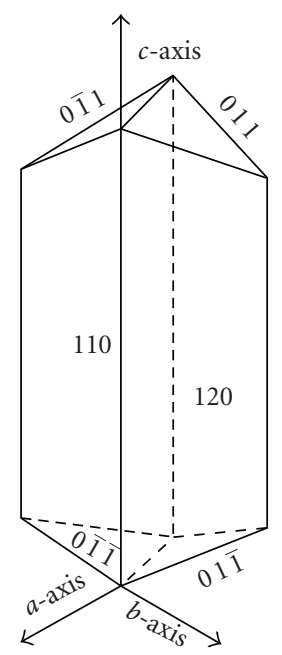

FIGURE 11: Morphology of L-threonine single crystal.

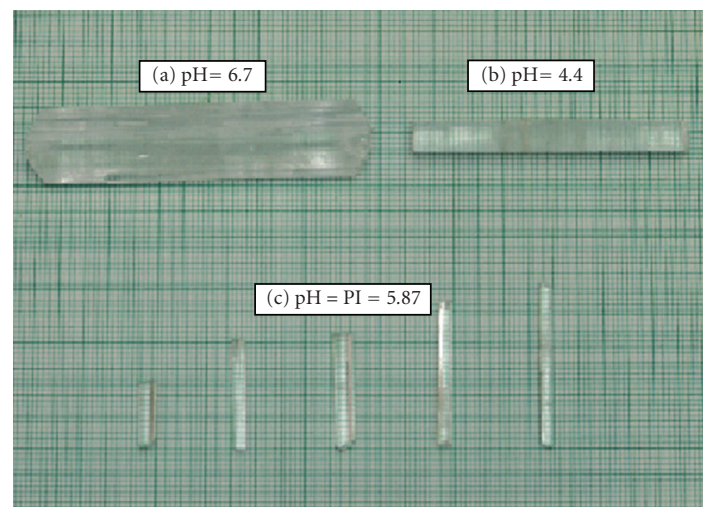

Figure 12: Photograph of L-threonine crystal grown at different $\mathrm{pH}$.

of cases $[41,42]$. For example, when the solution $\mathrm{pH}$ deviates from the stoichiometric $\mathrm{pH}$, the solubility increases in the case of KDP [43]. From the utility point of view, it is advantageous to grow the crystals at various $\mathrm{pH}$ values.

For the bulk growth of L-threonine single crystal at different $\mathrm{pH}$, slow cooling method was employed. For that, the $\mathrm{pH}$ of the mother solutions were adjusted and altered to $\mathrm{pH}$ $=4.4,5.87$, and 6.70 with the help of dilute acetic acid. Seed crystals were taken by slow evaporation. Precautive measure was taken to avoid fungal contamination of the solution by adding $10 \%$ ethyl alcohol in the growth vessel. Mother solutions of different $\mathrm{pH}$ were saturated at $45^{\circ} \mathrm{C}$ and allowed to cool at the rate of $0.1^{\circ} \mathrm{C} /$ day. Crystals of various $\mathrm{pH}$ have been harvested at different growth periods and hence the size of crystal varies. This has been clearly projected in Figure 12.

Crystals of $\mathrm{pH}=6.70,4.40$, and 5.87 have been grown at the growth run of 45,30 , and 20 days, respectively. The crystal grown at the characteristic pH (PI) called isoelectric point ( $\mathrm{pH}=\mathrm{PI}=5.87$ ) of L-threonine was found to be highly transparent when compared to crystals grown at $\mathrm{pH}=4.4$ and $\mathrm{pH}=6.7$. The transparency variation with $\mathrm{pH}$ could easily be seen from the photograph shown.

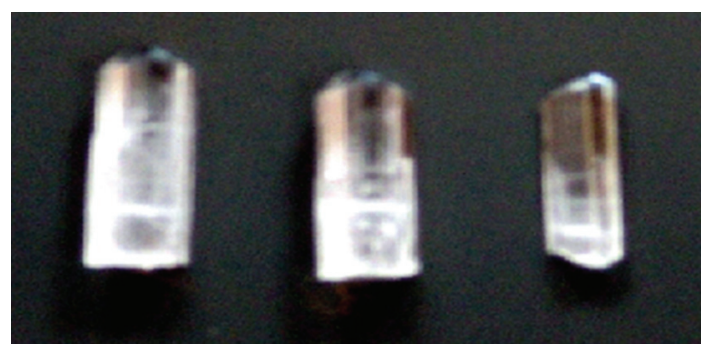

Figure 13: Deuterated L-threonine single crystals grown from seed rotation technique.

2.4. Crystal Growth of Deuterated L-Threonine. Seed crystals of deuterated L-threonine (D-LT) were taken after repeated recrystallization of L-threonine with $\mathrm{D}_{2} \mathrm{O}(99.9 \%)$. The degree of deuteration can be determined qualitatively through Fourier transform infrared spectroscopy (FTIR). Only the $\mathrm{NH}$ and $\mathrm{OH}$ protons are replaced by deuterium and the protons of $\mathrm{CH}$ and $\mathrm{CH}_{3}$ are unaffected. The deuteration of the $\mathrm{OH}$ and $\mathrm{NH}$ sites can be brought arbitrarily close to $100 \%$ by multiple recrystallizations of L-threonine salt with fresh $\mathrm{D}_{2} \mathrm{O}$.

Using the deuterated L-threonine salt and $\mathrm{D}_{2} \mathrm{O}$, the crystal growth proceeds by employing slow cooling technique with the help of deuterated L-threonine (D-LT) seed crystal of well-defined morphology. The deuterated solution was saturated to a desired temperature. The seed crystal suspended in the solution was allowed to rotate at low rpm (4-10 rpm). Crystals of dimensions $15 \times 6 \times 2 \mathrm{~mm}^{3}$ have been grown after a typical growth period of 6-7 days and are shown in Figure 13. The morphology of the deuterated crystal is almost similar to normal L-threonine. No new crystal face was observed in the grown crystal.

2.5. Evaluation of Second-Order NLO Coefficients Using ThreeDimensional Anharmonic Oscillator (AHO) Model. Optical effects in matter result from the polarization of the electrons in the medium in response to the electromagnetic field associated with the light propagating the medium. A simple model for these interactions is the Lorentz oscillator described in a variety of book sources. Here an electron is bonded to a nucleus by a spring with a natural frequency $\omega_{o}$ and equilibrium displacement " $x$ ". The electric component of the optical field felt by the electron is represented as a sinusoidally varying field. Considering only the linear response of this object, the equation of motion using harmonic oscillator approximation can be written as

$$
\frac{d^{2} x}{d t^{2}}+\Gamma \frac{d x}{d t}+\omega_{o}^{2} x=-\left(\frac{e}{m_{e}}\right) E_{x}
$$

where $\Gamma$ is damping constant e-electronic charge; $\omega_{o}$ is electronic resonance frequency.

Solving (16) the linear susceptibility is given by

$$
\chi^{1}(\omega)=\frac{N e^{2}}{\left[m_{e} \varepsilon_{o}\left(\omega_{o}^{2}-\omega^{2}-j \omega \Gamma\right)\right]} .
$$


Neglecting the damping term for the range of wavelength is considered, then we have

$$
\chi^{1}(\omega)=\frac{N e^{2}}{\left[m_{e} \varepsilon_{o}\left(\omega_{o}^{2}-\omega^{2}\right)\right]} .
$$

For the present study, we have considered anharmonic oscillator model first introduced by Bloembergen [44]. Using this model, the expressions for the second order nonlinear susceptibilities such as linear electro-optic effect and the second harmonic generation are described by adding anharmonic terms in the first equation.

Bloembergen's anharmonic oscillator model was well established and modulated by many authors [45-47]. Akitt et al. [48] utilized two coupled anharmonic oscillators to predict the nonlinear susceptibilities for a simple cubic material. The nonlinearities are assumed to be due to bound electrons and the fundamental ionic lattice resonance and their mutual interactions. The model predicts nonlinear susceptibilities in terms of the linear susceptibilities and four constants, the constants being anharmonic potential coefficients included as the perturbations. With the constants fixed, the SHG susceptibility as a function of frequency is calculated by using the formulae keeping $\omega_{1}=\omega_{2}=\omega$ as

$$
\begin{aligned}
\chi^{(2)}(2 \omega, \omega,, \omega) \\
=-\left(\frac{\varepsilon_{o}^{2}}{2}\right) \\
\times\left\{3\left[\frac{\chi_{e}^{1}(\omega) \chi_{e}^{1}(\omega) \chi_{e}^{1}(2 \omega)}{N_{e}^{2} e_{e}^{3}}\right] \mathbf{D}\right. \\
+\left[\frac{\chi_{e}^{1}(\omega) \chi_{e}^{1}(\omega) \chi_{i}^{1}(2 \omega)}{N_{e}^{2} e_{e}^{2} e_{i}}+\frac{2 \chi_{e}^{1}(\omega) \chi_{i}^{1}(\omega) \chi_{i}^{1}(2 \omega)}{N_{e} e_{e}^{2} N_{i} e_{i}}\right] \mathbf{C} \\
+\left[\frac{2 \chi_{e}^{1}(\omega) \chi_{i}^{1}(\omega) \chi_{i}^{1}(2 \omega)}{N_{e} N_{i} e_{e} e_{i}^{2}}+\frac{\chi_{i}^{1}(\omega) \chi_{i}^{1}(\omega) \chi_{e}^{1}(2 \omega)}{N_{i}^{2} e_{i}^{2} e_{e}}\right] \mathbf{B} \\
\left.+\left[\frac{3 \chi_{i}^{1}(\omega) \chi_{i}^{1}(\omega) \chi_{i}^{1}(2 \omega)}{N_{i}^{2} e_{i}^{3}}\right] \mathbf{A}\right\} .
\end{aligned}
$$

The susceptibility for the electro-optic effect is obtained for $\omega_{1}$ at optical frequency, $\omega_{2} \simeq 0$ and it is given by

$$
\begin{aligned}
& \chi^{(2)}(\omega, \omega, 0) \\
&=-\varepsilon_{o}^{2}\left\{\left[3\left[\frac{\chi_{e}^{1}(\omega) \chi_{e}^{1}(\omega) \chi_{e}^{1}(0)}{N_{e}^{2} e_{e}^{3}}\right] \mathbf{D}\right.\right. \\
&+\left[\frac{\chi_{e}^{1}(\omega) \chi_{i}^{1}(\omega) \chi_{e}^{1}(0)}{N_{e}^{2} e_{e}^{2} e_{i}}+\frac{\chi_{e}^{1}(\omega) \chi_{e}^{1}(\omega) \chi_{i}^{1}(0)}{N_{e} e_{e}^{2} N_{i} e_{i}}\right. \\
&\left.+\frac{\chi_{e}^{1}(\omega) \chi_{i}^{1}(\omega) \chi_{e}^{1}(0)}{N_{e} N_{i} e_{e}^{2} e_{i}}\right] \mathbf{C}+\frac{\left[\chi_{e}^{1}(\omega) \chi_{i}^{1}(\omega) \chi_{i}^{1}(0)\right]}{N_{e} N_{i} e_{e} e_{i}^{2}} \\
&\left.+\frac{\chi_{e}^{1}(\omega) \chi_{i}^{1}(\omega) \chi_{i}^{1}(0)}{N_{i}^{2} e_{i}^{2} e_{e}}+\frac{\chi_{i}^{1}(\omega) \chi_{i}^{1}(\omega) \chi_{e}^{1}(0)}{N_{i} N_{e} e_{i} e_{e}^{2}}\right] \mathbf{B} \\
&\left.+\left[\frac{3 \chi_{i}^{1}(\omega) \chi_{i}^{1}(\omega) \chi_{i}^{1}(2 \omega)}{N_{i}^{2} e_{i}^{3}}\right] \mathbf{A}\right\},
\end{aligned}
$$

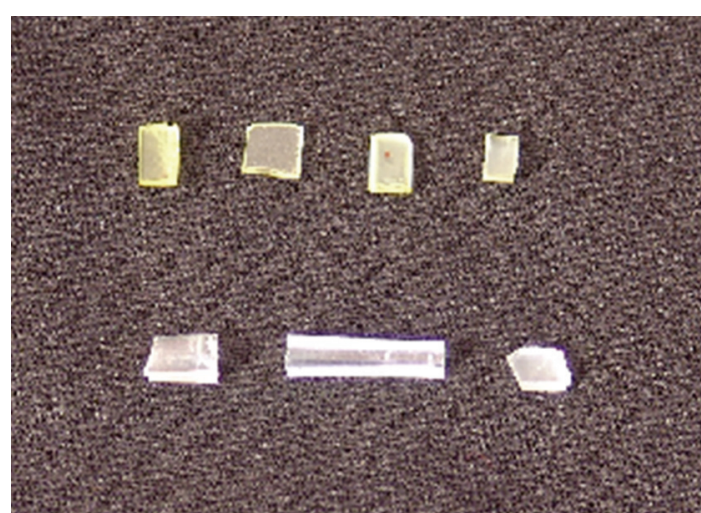

FIGURE 14: Photograph of electron irradiated L-threonine for various fluences.

where $\chi_{e}^{1}(\omega)$ and $\chi_{i}^{1}(\omega)$ are linear susceptibilities due to electronic and ionic polarizabilities. $N_{i}$ and $N_{e}$ are the number of electronic and ionic oscillators per unit cube. A, B, C, and D are constants referred to as nonlinear strength factors [49]. Sugie and Tada [50] first gave the threedimensional AHO model and it is equivalent to Garrett's [45] one-dimensional approach. They measured the linear electro-optic coefficients of GaAs and CdS and deduced the anharmonic potential coefficients using their model. Ramesh and Srinivasan [51] have also used three-dimensional two coupled anharmonic oscillator model and calculated optical nonlinearities for wide band gap crystals.

The primary shortcoming of the classical model of optical nonlinearities presented here is that this model describes a single resonance frequency for electronic $\left(\omega_{e}\right)$ and ionic $\left(\omega_{i}\right)$ polarizations to each atom. In contrast, the quantum mechanical theory of nonlinear optics allows each atom to possess many energy eigen-values and hence more than one resonance frequency. Since the present model allows for only one resonance frequency, it cannot properly describe the complete resonance nature of the nonlinear susceptibility such as one and two photon resonances. However, it provides a good description for those cases in which all of the optical frequencies are considerably smaller than the lowest electronic resonance frequency of the material system. As the electronic resonance frequency of the L-threonine crystal lie under this category, this model has been chosen to describe its nonlinear optical properties.

2.6. Electron Irradiation on L-Threonine Single Crystals. Good quality crystals were cut and polished using alumina abrasive to the dimensions of $5 \times 5 \times 1 \mathrm{~mm}^{3}$. The samples were irradiated by a $6 \mathrm{MeV}$ electron beam using the Race-Track microtron facility of Pune University, India. Irradiations of the crystals were done at different electron fluences, namely, $1.5 \times 10^{14}, 3 \times 10^{14}, 6 \times 10^{14}, 9 \times 10^{14}$ and $12 \times 10^{14} \mathrm{e} / \mathrm{cm}^{2}$. After irradiation the samples turn yellowish due to the formation of coulour centres, that is, electronic defects produced in gap along the particle track [52] and the photograph of the irradiated crystals is shown in Figure 14.

2.7. Ion Irradiation on L-Threonine Single Crystals. Crystals with well-defined morphology were cut into pieces of 


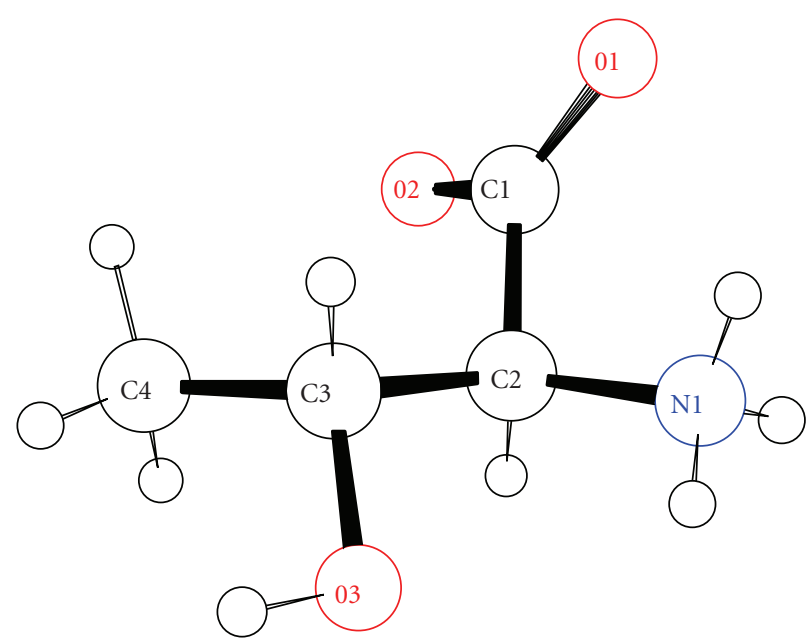

FIGURE 15: Molecular arrangement of L-threonine molecule.

TABLE 2: Crystallographic details of L-threonine.

\begin{tabular}{|c|c|}
\hline Molecular formula & $\mathrm{C}_{4} \mathrm{H}_{9} \mathrm{NO}_{3}$ \\
\hline Unit cell parameters & $\begin{array}{l}a=13.611 \AA, b=7.738 \AA \text { and } c=5.144 \AA \\
\alpha=\beta=\gamma=90^{\circ}\end{array}$ \\
\hline Cell volume & $537.98 \AA^{3}$ \\
\hline System & Orthorhombic \\
\hline Space group & $\mathrm{P} 2_{1} 2_{1} 2_{1}\left(\mathrm{D}_{2}^{4}\right)$ \\
\hline Formula weight & $119 \mathrm{~g} / \mathrm{mol}$ \\
\hline Density & $1.45 \mathrm{~g} / \mathrm{cc}$ \\
\hline
\end{tabular}

dimensions of $5 \times 5 \times 1 \mathrm{~mm}^{3}$ and polished using alumina paste. $\mathrm{Li}^{3+}$ ions of $50 \mathrm{MeV}$ from the $15 \mathrm{UD}$ pelletron [53] at Inter University Accelerator Centre (IUAC) New Delhi, India were allowed to incident on the sample mounted inside a $1.5 \mathrm{~m}$ diameter scattering chamber. The incident beam current was about 2 particle nano amperes (pna). The vacuum in the chamber during the experiment was kept at $8 \times 10^{-7}$ mbar. The crystals have been irradiated at various ion fluences, namely, $1 \times 10^{10}, 1 \times 10^{11}$ and $1 \times$ $10^{12} \mathrm{Li}^{3+}$ ions $/ \mathrm{cm}^{2}$ so as to study the fluence dependence on various physiochemical properties.

\section{Results and Discussions}

3.1. Crystallographic Details of L-Threonine. The unit cell dimensions and the space group of L-threonine were obtained using a single crystal X-ray diffractometer equipped with $\mathrm{CuK} \alpha$ radiation $(\lambda=1.54598 \AA)$. Lattice parameters were calculated from 25 reflections. The crystal data of Lthreonine is presented in Table 2. Molecular structure of Lthreonine has been projected through ORTEP representation and it is shown in Figure 15.

3.2. Characterization Studies. FTIR spectrum of L-threonine was recorded using Bruker IFS 66 FT-IR spectrometer by $\mathrm{KBr}$ pellet method and FT-Raman measurement of the crystal was also performed in the same instrument with the

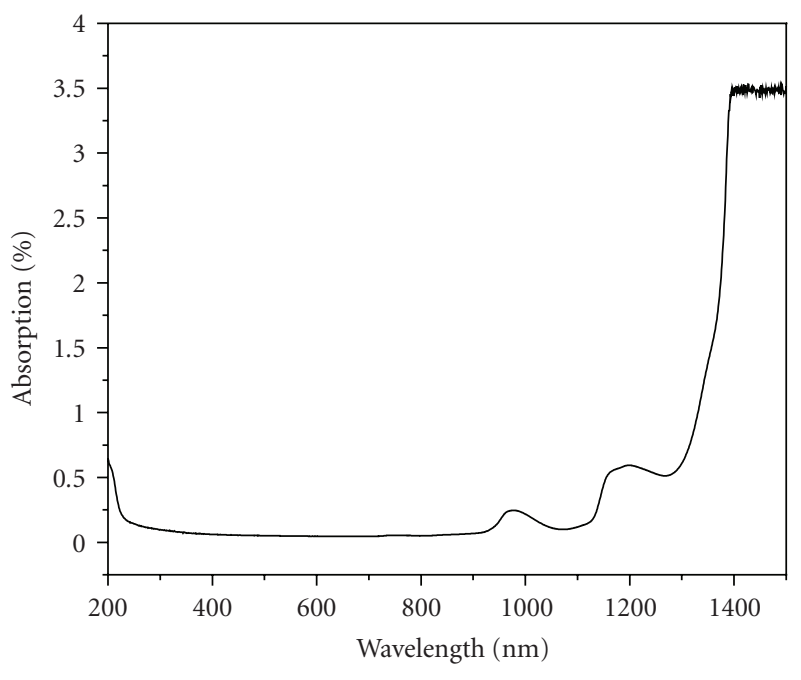

FIGURE 16: UV-Vis-NIR spectrum of L-threonine

Raman attachment. The transmission range of L-threonine crystal was measured in the range $200-1500 \mathrm{~nm}$ using Varian Cary 5E UV-Vis-NIR spectrophotometer. Dielectric measurement was carried out for L-threonine single crystal in the frequency range $1 \mathrm{kHz}-1 \mathrm{MHz}$ using a Solartron impedence analyzer. Thermomechanical analysis (TMA) was performed for the crystals in the temperature range $50-130^{\circ} \mathrm{C}$ using Mettler TA 3000 TMA analyzer. Thermo gravimetric analysis (TGA) was done using Netzsch STA 409 analyzer in nitrogen atmosphere at a heating rate of $10^{\circ} \mathrm{C} / \mathrm{min}$ and differential Scanning Calorimetry (DSC) was carried out using Netzsch DSC 204 in nitrogen atmosphere at a heating rate of $10^{\circ} \mathrm{C} / \mathrm{min}$. The second order NLO property of L-threonine crystal was tested by Kurtz-powder SHG test using a Nd : YAG laser (1064 nm).

3.2.1. UV-Vis-NIR Spectral Studies. The UV-Vis-NIR spectrum recorded for L-threonine in the wavelength range 200$1500 \mathrm{~nm}$ is represented in Figure 16.

The transparency $(\sim 85 \%)$ region lies in the range 250 $900 \mathrm{~nm}$ and the UV transparency lower cutoff of L-threonine occurs at $220 \mathrm{~nm}$ [54]. From the spectrum, it has been inferred that there is a minimum absorption in the whole of the visible region and hence L-threonine can be feasible candidate for SHG applications in the visible region.

3.2.2. FTIR and FT-Raman Spectroscopy. FTIR and FTRaman spectra of L-threonine were recorded at room temperature in the frequency range $400-4000 \mathrm{~cm}^{-1}$ and $50-3500 \mathrm{~cm}^{-1}$ and it is shown in Figure 17. In the FTIR spectrum the band observed at $489 \mathrm{~cm}^{-1}$ is assigned to torsional mode of $\mathrm{NH}_{3}$. The band at $701 \mathrm{~cm}^{-1}$ is assigned to the wagging vibration of $\mathrm{CO}_{2}{ }^{-}$structure. Torsion of $\mathrm{COH}$ structure is observed around $750 \mathrm{~cm}^{-1}$. The bending of $\mathrm{CO}_{2}{ }^{-}$is observed at $767 \mathrm{~cm}^{-1}$. Band at $931 \mathrm{~cm}^{-1}$ is associated with CC-stretching vibration. Due to the stretching vibration involving carbon and nitrogen of the amino group there exists a peak at $1040 \mathrm{~cm}^{-1}$. 


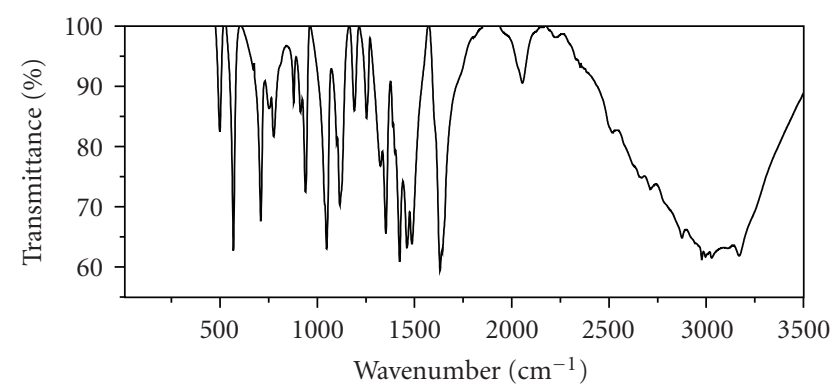

- FTIR

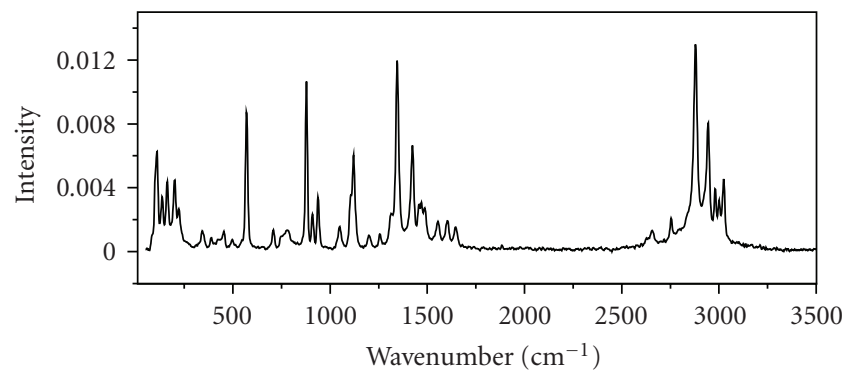

— Raman

(a)

(b)

FIGURE 17: FTIR and FT-Raman spectra of L-threonine.

The rocking of $\mathrm{NH}_{3}{ }^{+}$structure is observed at $1184 \mathrm{~cm}^{-1}$. Bending vibrations of $\mathrm{CH}$ group are found in L-threonine with peaks at $1246,1318,1346 \mathrm{~cm}^{-1}$ in the spectrum. Bending vibrations of the $\mathrm{CH}_{3}$ group is assigned to the peak at $1457 \mathrm{~cm}^{-1}$. The symmetric stretching of $\mathrm{CO}_{2}{ }^{-}$structure is found at $1417 \mathrm{~cm}^{-1}$ in the IR spectrum. The symmetric bending of $\mathrm{NH}_{3}{ }^{+}$is observed at $1481 \mathrm{~cm}^{-1}$. The band at $1623 \mathrm{~cm}^{-1}$ may be assigned to the asymmetric stretching $\mathrm{CO}_{2}{ }^{-}$. The stretching vibrations also exist in both the $\mathrm{NH}_{3}{ }^{+}$ and $\mathrm{CH}$ structures. The symmetric stretching vibrations of $\mathrm{NH}_{3}{ }^{+}$may be assigned to the vibration at $3021 \mathrm{~cm}^{-1}$. The asymmetric stretching of the $\mathrm{NH}_{3}{ }^{+}$is observed at wave number $3157 \mathrm{~cm}^{-1}$.

In the Raman spectrum, the $\mathrm{CH}_{3}$ vibrations at 2944 and $2879 \mathrm{~cm}^{-1}$ appear as intense peaks in the higher energy region. The sharp peak at $3024 \mathrm{~cm}^{-1}$ is assigned to hydrogen bonding $\mathrm{NH}$ vibrations. The less intense peaks at 1645,1423 , and $1554 \mathrm{~cm}^{-1}$ are assigned to stretching vibrations of $\mathrm{CO}_{2}{ }^{-}$ and $\mathrm{NH}$ bending. The $\mathrm{CH}_{3}$ bending vibrations produce a characteristic peak at $1454 \mathrm{~cm}^{-1}$. The intense peaks at 877 and $570 \mathrm{~cm}^{-1}$ are due to $\mathrm{C}-\mathrm{C}-\mathrm{N}$ and $\mathrm{CO}_{2}{ }^{-}$rocking respectively. The remaining vibrations in both FTIR and FTRaman spectra are tabulated in Table 3 . The peak positions are in good agreement with earlier reports $[55,56]$.

3.2.3. Thermal Analysis. The thermal strength of the crystal has been investigated through differential scanning calorimetry in the temperature range $-100^{\circ} \mathrm{C}$ to $300^{\circ} \mathrm{C}$ at a heating rate of $5^{\circ} \mathrm{C} / \mathrm{min}$ and thermogravimetric analysis in the temperature $30^{\circ} \mathrm{C}$ to $500^{\circ} \mathrm{C}$ at a heating rate of $5^{\circ} \mathrm{C} / \mathrm{min}$ are shown in Figures 18(a) and 18(b), respectively. From the DSC curve, it is clear that no anomalous behaviour (phase transition) was observed in both lower and higher temperature region. It is worthwhile to note that some kind of structural instability has been found out around $-30^{\circ} \mathrm{C}$ to $-33^{\circ} \mathrm{C}(\Delta \mathrm{Cp} \simeq 0.01 \mathrm{~W} / \mathrm{g})$ in similar neutral amino acid, $\mathrm{L}-$ alanine single crystal [57]. But such a possibility of second order structural phase transition is ruled out to our case as the presented compound shows a single endothermic peak and a sudden weight loss at $265.2^{\circ} \mathrm{C}$ in DSC and TGA correspond to the decomposition temperature of Lthreonine molecule.
TABLe 3: Bands assignments of IR and Raman spectra of Lthreonine.

\begin{tabular}{|c|c|c|}
\hline \multicolumn{2}{|c|}{ Wavenumber $\left(\mathrm{cm}^{-1}\right)$} & \multirow[b]{2}{*}{ Assignments } \\
\hline IR & Raman & \\
\hline \multirow[t]{2}{*}{3157,3021} & 3024 & $\mathrm{~N}-\mathrm{H}$ stretch \\
\hline & 2944 & Asymmetric stretching of $\mathrm{CH}_{3}$ \\
\hline \multirow[t]{2}{*}{2975} & 2935 & $\mathrm{C}-\mathrm{H}$ stretching \\
\hline & 2879 & Symmetric stretching of $\mathrm{CH}_{3}$ \\
\hline 2050 & & $\begin{array}{l}\text { Degenerative deformation of } \mathrm{NH}_{3}{ }^{+} \text {and } \\
\text { torsional vibration of } \mathrm{NH}_{3}{ }^{+}\end{array}$ \\
\hline 1623 & 1645 & Asymmetric stretching of $\mathrm{CO}_{2}^{-}$ \\
\hline 1481 & 1544 & Bending vibration of $\mathrm{NH}_{3}^{+}$ \\
\hline \multirow[t]{2}{*}{1455} & & Degenerative deformation of $\mathrm{CH}_{3}$ \\
\hline & 1454 & Bending vibration of $\mathrm{CH}_{3}$ \\
\hline 1417 & 1423 & Symmetric stretching of $\mathrm{CO}_{2}$ \\
\hline \multirow[t]{2}{*}{$\begin{array}{l}1246, \\
1318,1346\end{array}$} & & In plane deformation of $\mathrm{C}-\mathrm{C}-\mathrm{H}$ \\
\hline & 1308 & Stretching of $\mathrm{C}-\mathrm{O}$ and bending of $\mathrm{O}-\mathrm{H}$ \\
\hline 1184 & & $\mathrm{NH}_{3}^{+}$rocking \\
\hline 1109 & 1101,1045 & $\mathrm{CH}_{3}$ rocking vibration \\
\hline \multirow[t]{2}{*}{931} & & $\mathrm{C}-\mathrm{C}$ stretching \\
\hline & 877 & $\mathrm{C}-\mathrm{C}-\mathrm{N}$ vibration \\
\hline 767 & & Out of plane bending vibration of $\mathrm{CO}_{2}^{-}$ \\
\hline \multirow[t]{2}{*}{701} & & In plane deformation of $\mathrm{CO}_{2}^{-}$ \\
\hline & 704 & Bending vibration of $\mathrm{CO}_{2}$ \\
\hline \multirow[t]{2}{*}{489} & 570 & $\mathrm{CO}_{2}$ rocking \\
\hline & 449 & Torsion vibration of $\mathrm{NH}_{3}{ }^{+}$ \\
\hline
\end{tabular}

3.2.4. Dielectric Measurements. Dielectric studies were performed for the crystal from the room temperature to $150^{\circ} \mathrm{C}$. Rectangular shaped crystal of dimension $(l=3.35 \mathrm{~mm}, b=$ $4.45 \mathrm{~mm}$ and $t=1.25 \mathrm{~mm}$ ) has been pasted with conductive silver paint for metallic contacts. Dielectric constant and dielectric loss $(\tan \delta)$ values have been found in the frequency range $1 \mathrm{KHz}-1 \mathrm{MHz}$. It was found that at all temperatures there was an increase in the dielectric constant $\left(\varepsilon_{r}\right)$ at low frequencies. This can be attributed to space-charge 


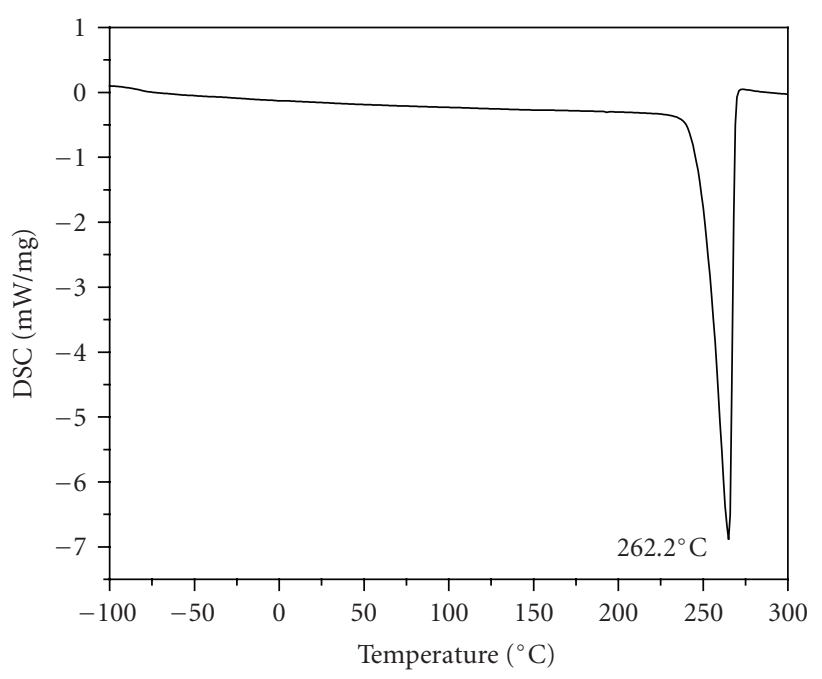

(a)

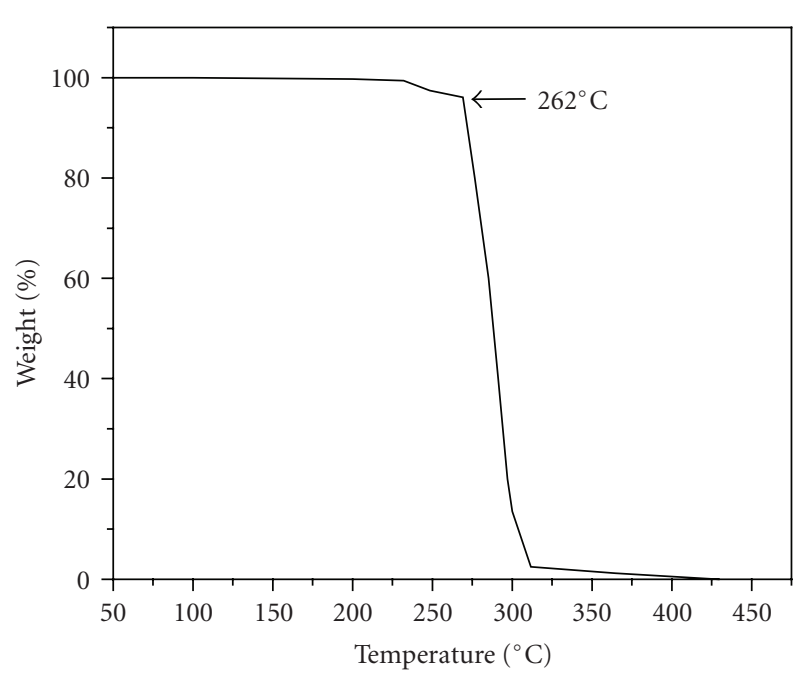

(b)

FIguRE 18: (a) DSC curve of L-threonine. (b) TGA curve of L-threonine.

polarization mechanism of molecular dipoles. After that the dielectric permittivity of the compound remains almost constant in the range 135-180 in the higher temperature range. The dielectric constants were found to be 61 and 175 at $1 \mathrm{MHz}$ for 25 and $110^{\circ} \mathrm{C}$ [58]. It is important to note that temperature has influenced much on the dielectric behaviour of the crystals at higher frequencies. Similarly the dielectric loss value increases at lower frequencies and this may be due to the fact that the natural frequency of the molecular dipoles matches with frequency of the applied a.c. field and thus causes the dissipation of energy in the form of heat. As expected the dielectric loss decreases at higher frequencies and it was found to have negligible values at all temperatures. The results have been presented in Figures 19(a) and 19(b), respectively.

3.2.5. Thermomechanical Analysis (TMA). The thermal expansion data of nonlinear optical crystals are important for the following reason. Upon irradiation with the laser beam, the optical absorption of the NLO material causes thermal gradients that disturb its phase matching properties. Thermal gradients can also lead to fractures if they are high enough. Hence, the thermomechanical analysis (Dialtometry) was carried in the temperature range $50-130^{\circ} \mathrm{C}$ at a heating rate of $5^{\circ} \mathrm{C} / \mathrm{min}$ in air atmosphere for L-threonine single crystals. Since the title compound crystallizes in an orthorhombic system, the principal expansion coefficient tensor coincides with the crystallographic axes [59] and the lengths $l_{1}=3.325 \mathrm{~mm}, l_{2}=2.542 \mathrm{~mm}$, and $l_{3}=$ $2.308 \mathrm{~mm}$ have been regarded as $c, b$, and $a$-axes of the crystal, respectively.

The thermal expansion tensor or strain tensor can be written in the form

$$
\varepsilon_{i j}=\alpha_{i j} \Delta T,
$$

where $\varepsilon_{i j}$ are constants of coefficients of thermal expansion and $\Delta T$ is the heating rate
In terms of principle axis, the strain tensor can be represented as

$$
\begin{aligned}
& \varepsilon_{1}=\alpha_{1} \Delta T, \\
& \varepsilon_{2}=\alpha_{2} \Delta T, \\
& \varepsilon_{3}=\alpha_{3} \Delta T,
\end{aligned}
$$

where $\alpha_{1}, \alpha_{2}$, and $\alpha_{3}$ are coefficients of thermal expansion along mutually perpendicular $c, b$, and $a$ axes. So, equation of the strain ellipsoid is given by

$$
\left[\frac{\left(X_{1}\right)^{2}}{\left(1+\varepsilon_{1}\right)^{2}}\right]+\left[\frac{\left(X_{2}\right)^{2}}{\left(1+\varepsilon_{2}\right)^{2}}\right]+\left[\frac{\left(X_{3}\right)^{2}}{\left(1+\varepsilon_{3}\right)^{2}}\right]=1 .
$$

The representation quadric for thermal expansion of the grown crystal has been projected along $a$-axis and it is shown in Figure 20.

The mean thermal expansion tensor $\alpha_{i j}$ of L-threonine crystal from Figure 21 is given by

$$
\alpha=\left[\begin{array}{ccc}
4.064 & 0 & 0 \\
0 & 3.0856 & 0 \\
0 & 0 & 1.944
\end{array}\right] \times 10^{-5} /{ }^{\circ} \mathrm{C},
$$

which corresponds to $c, b$, and $a$-axes and the strain tensor $\left(\varepsilon_{i j}\right)$ is given by

$$
\begin{aligned}
& \varepsilon_{3}=9.722 \times 10^{-5}, \\
& \varepsilon_{2}=15.428 \times 10^{-5}, \\
& \varepsilon_{1}=20.319 \times 10^{-5} .
\end{aligned}
$$

The bulk expansion coefficient is given by

$$
\alpha_{v}=\alpha_{1}+\alpha_{2}+\alpha_{3},
$$

and it was found as $9.096 \times 10^{-5} /{ }^{\circ} \mathrm{C}$. 


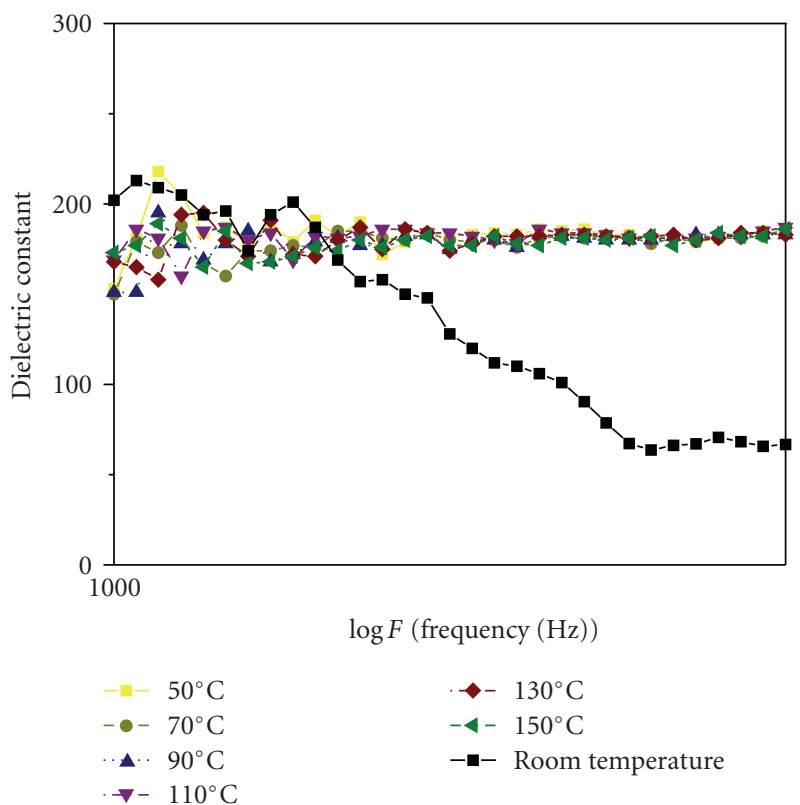

(a)

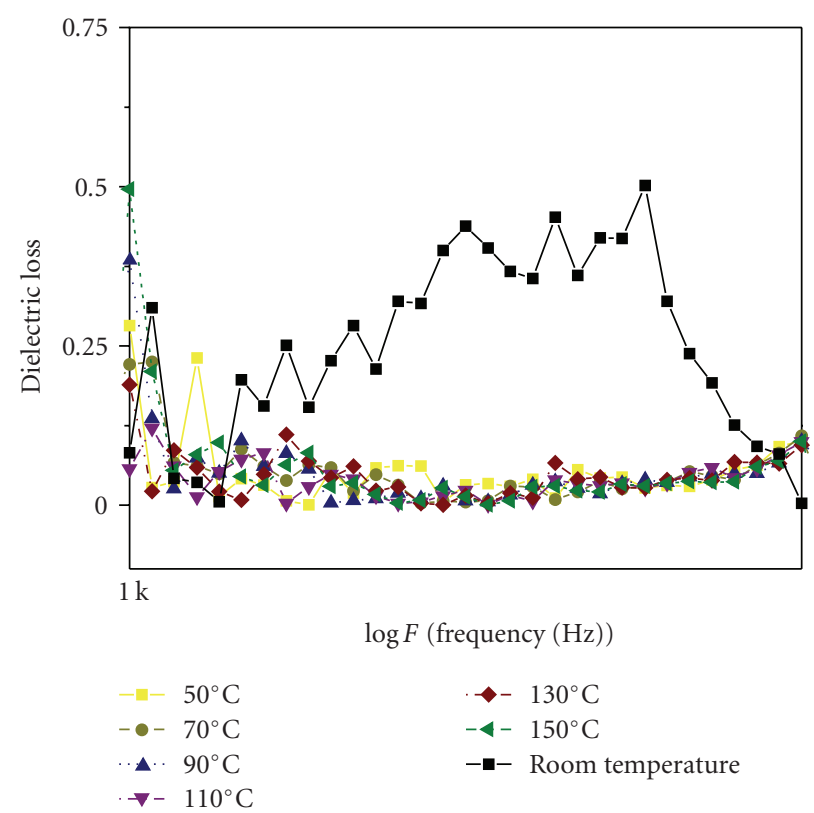

(b)

Figure 19: (a) Dielectric constant of L-threonine. (b) Dielectric loss of L-threonine.

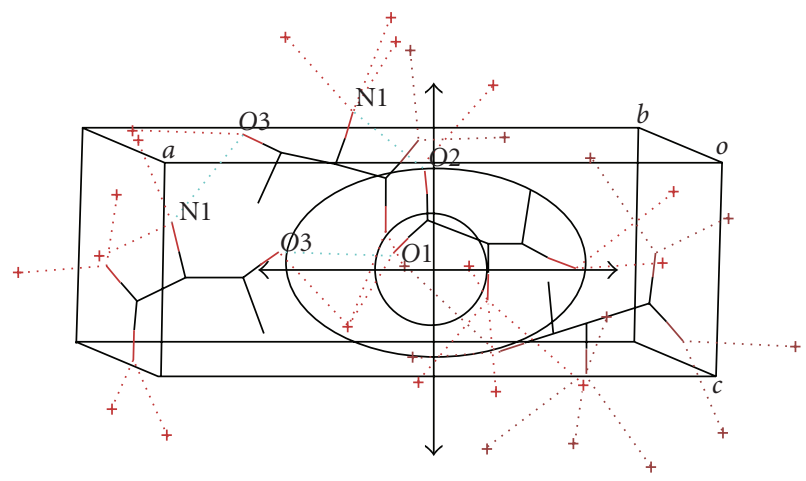

Figure 20: Thermal expansion projection of L-threonine along $a$ axis.

Figures 21, 22, 23, and 24 show the anisotropy of linear expansion coefficient $(\alpha)$, bulk expansion coefficient $\left(\alpha_{v}\right)$, linear expansion $(\Delta L)$, and linear strain as a function of temperature.

From Figure 24, it was found that no negative thermal expansion has been observed in L-threonine single crystal in all mutually perpendicular crystallographic axes. The coefficients of thermal expansions were comparable to that of L-alanine single crystals [60]. L-threonine crystal shows large expansion coefficient along $c$-axis when compared to $\mathrm{b}$ and $a$-axes. The anisotropy in the expansion coefficients can be perceived from the hydrogen bonding in L-threonine.

According to crystal data [61], 4 hydrogen bonds are possible for the crystal, out of which three are of $\mathrm{N}-\mathrm{H} \cdots \mathrm{O}$ type and the remaining one form $\mathrm{O}-\mathrm{H} \cdots \mathrm{O}$ bonds. The crystallographic $b$ and $c$ axes are linked through a chain of $\mathrm{N}-\mathrm{H}$. . O bonds while $a$-axis is linked by a chain of O$\mathrm{H}$. . O bonds. The higher expansion coefficient along c-axis

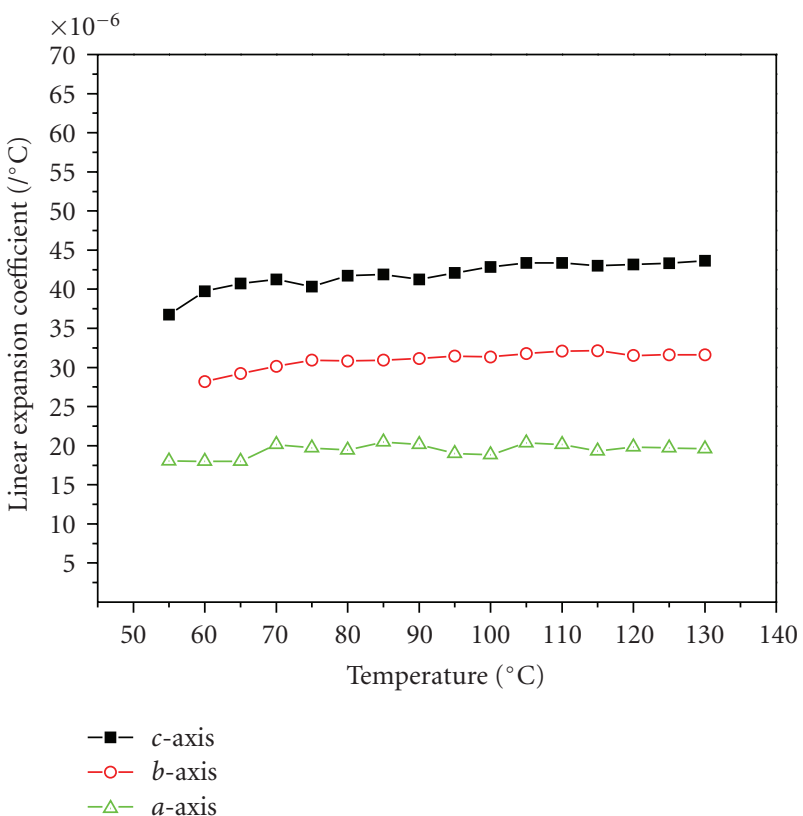

FIGURE 21: Anisotropy of linear expansion coefficient along the three axis.

can be attributed to longer intramolecular contact $(2.917 \AA)$ and hence the bond is weak in nature when compared to $\mathrm{O}-$ $\mathrm{H}$. . O bond whose bond length is $2.660 \AA$. It is obvious that the increase in thermal expansion in $b$ and $c$-axes is due to the flexible and weak intermolecular $\mathrm{N}-\mathrm{H}$. . O bonding. The above observation may also be helpful in understanding the intermolecular forces and the bonding nature in the crystal structure of L-threonine. Thermal expansion measurements were also helpful to investigate the cracking problem of the 


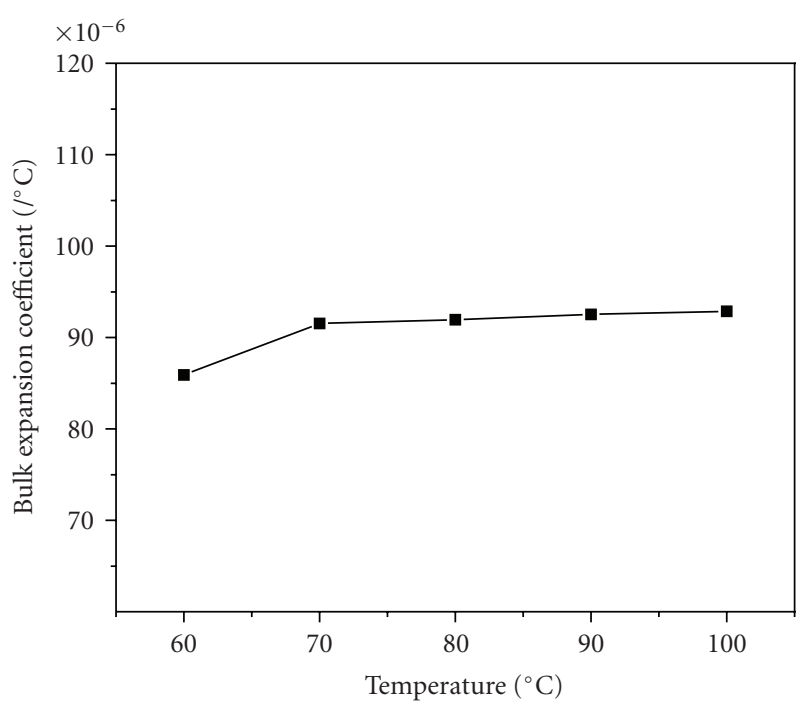

FiguRE 22: Bulk expansion coefficient versus temperature.

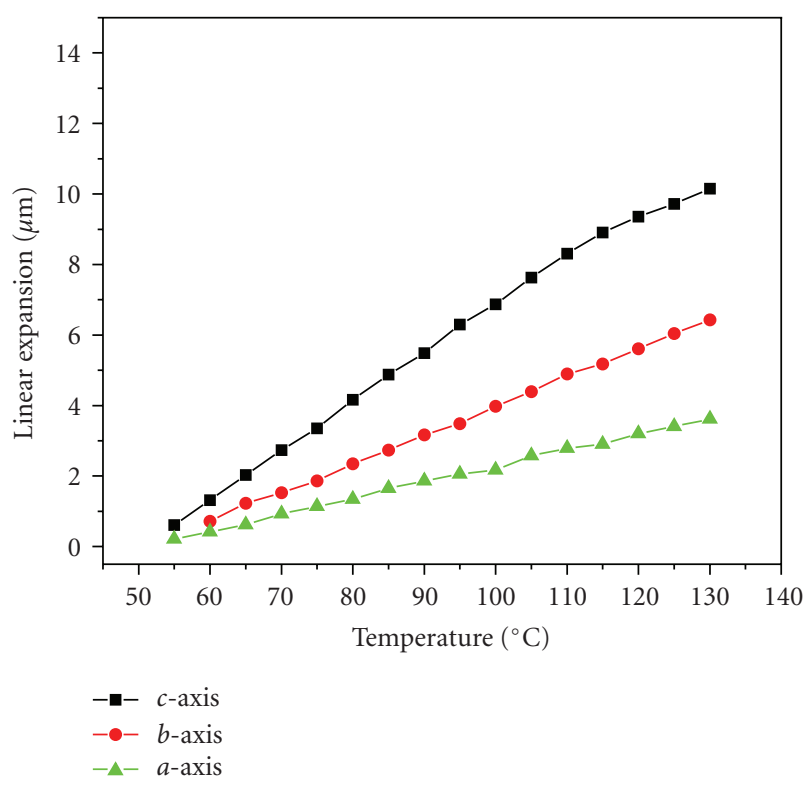

FIgURE 23: Anisotropy of linear expansion along the three axis.

crystal. During bulk crystal growth, temperature gradient is necessarily present in the crystal and additional gradients are superposed transiently in slow cooling technique. This may be a one among the reasons why cracks or additive polycrystals sometimes appear during the growth process.

Thermal gradients give rise to the thermal stresses whose magnitude is given by $\sigma=Y \alpha \Delta T$, where $Y$ is the elastic modulus. It is known that the fracture temperature is inversely proportional to thermal expansion coefficient [62] and L-threonine crystal also shows small expansion coefficient along the three axes; so the material can absorb more energy while maintaining a smaller extension and makes it difficult to produce crack.

The expansion coefficients of L-threonine were found to be less when compared to many novel NLO crystals. This

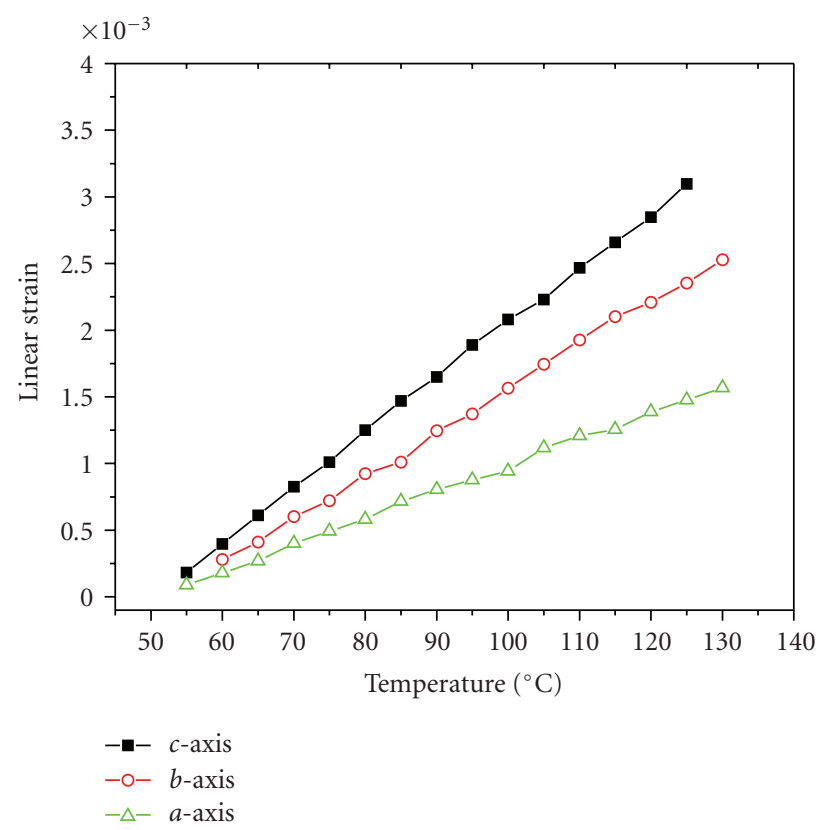

FIgURE 24: Anisotropy of linear strain along the three axis.

probably makes the crystal feasibility to be useful in electro optic Pockel's devices. Usually for manufacturing an optical waveguide, the typical length of the crystal wafer is about $1 \mathrm{~cm}$. The thermal expansion coefficients of L-threonine show that temperature variation of $1^{\circ} \mathrm{C}$ leads to a length variation of $0.3 \mu \mathrm{m}$ which is quite lower than the wavelengths of the conventional laser sources. This behaviour will be more important when one considers manufacturing devices such as waveguide and interferometer with L-threonine single crystals [58]. A comparative list on the thermal expansion coefficients of various NLO crystals has been given in Table 4.

3.2.6. Microhardness Measurements. Hardness is an important solid state property and plays a vital role in device fabrication and hence Vicker's microhardness measurement was carried out for L-threonine crystals to assess its mechanical strength. To evaluate the Vicker's hardness number, grown crystals of L-threonine were subjected to static indentation test at room temperature using Leitz Wetzlar hardness tester fitted with Vicker's diamond pyramidal indentor. Several indentations were made on the (lll $\left.\begin{array}{lll}1 & 2 & 0\end{array}\right)$ face along the $\left(\begin{array}{lll}0 & 0 & 1\end{array}\right)$ of L-threonine crystal. The Vicker's hardness number was calculated using the expression

$$
H_{v}=1.8544\left(\frac{P}{d_{2}}\right)
$$

where $H_{v}$ is the Vicker's hardress number for a given load, $P$ in grams, and " $d$ " is the average diagonal length of the indentation in $\mathrm{mm}$. At lower loads there is an increase in the hardness number which can be attributed to the electrostatic attraction between the Zwitterions present in the molecule. This favours all amino acids for their good mechanical strength [63]. 
TABLE 4: Comparative data on thermal expansion coefficient values of various novel NLO crystals.

\begin{tabular}{|c|c|c|c|}
\hline S.No & Compound & System & Thermal expansion coefficient $\left(\alpha /{ }^{\circ} \mathrm{C}\right)$ \\
\hline \multirow{3}{*}{ (1) } & \multirow{3}{*}{ L-threonine } & \multirow{3}{*}{ Orthorhombic } & $\alpha_{11}=4.06 \times 10^{-5}$ \\
\hline & & & $\boldsymbol{\alpha}_{2}=3.09 \times 10^{-5}$ \\
\hline & & & $\boldsymbol{\alpha}_{3}=1.94 \times 10^{-5}$ \\
\hline \multirow{3}{*}{ (2) } & \multirow{3}{*}{ Deuterated L-arginine Phosphate } & \multirow{3}{*}{ Monoclinic } & $\alpha_{1}=5.74 \times 10^{-5}$ \\
\hline & & & $\alpha_{2}=0.80 \times 10^{-5}$ \\
\hline & & & $\alpha_{3}=1.83 \times 10^{-5}$ \\
\hline \multirow{3}{*}{ (3) } & \multirow{3}{*}{ Urea-(d) tartaric acid } & \multirow{3}{*}{ Orthorhombic } & $\alpha_{1}=5.23 \times 10^{-5}$ \\
\hline & & & $\alpha_{2}=3.86 \times 10^{-5}$ \\
\hline & & & $\alpha_{3}=3.57 \times 10^{-5}$ \\
\hline \multirow{2}{*}{ (4) } & \multirow{2}{*}{ Triallyl thiourea mercury bromide } & \multirow{2}{*}{ Trigonal } & $\alpha_{1}=2.83 \times 10^{-5}$ \\
\hline & & & $\alpha_{3}=7.98 \times 10^{-5}$ \\
\hline \multirow{3}{*}{ (5) } & \multirow{3}{*}{ Bismuth triborate } & \multirow{3}{*}{ Monoclinic } & $\alpha_{1}=4.81 \times 10^{-5}$ \\
\hline & & & $\alpha_{2}=4.40 \times 10^{-5}$ \\
\hline & & & $\alpha_{3}=-2.69 \times 10^{-5}$ \\
\hline \multirow{3}{*}{ (6) } & \multirow{3}{*}{ p-nitrophenolate dihydrate } & \multirow{3}{*}{ Orthorhombic } & $\alpha_{1}=7.48 \times 10^{-5}$ \\
\hline & & & $\alpha_{2}=0.43 \times 10^{-5}$ \\
\hline & & & $\alpha_{3}=1.99 \times 10^{-5}$ \\
\hline \multirow{2}{*}{ (7) } & \multirow{2}{*}{ Tri-allyl thiourea cadmium chloride } & \multirow{2}{*}{ Trigonal } & $\alpha_{1}=2.19 \times 10^{-5}$ \\
\hline & & & $\alpha_{3}=6.15 \times 10^{-5}$ \\
\hline
\end{tabular}

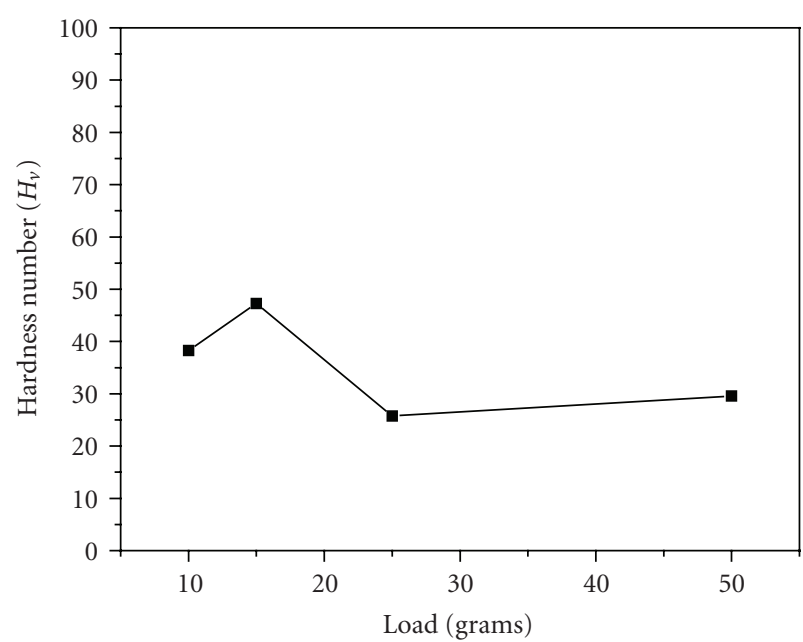

FIgURE 25: Microhardness versus Load for L-threonine.

Figure 25 shows the dependence of hardness on load for L-threonine crystal. At higher loads the hardness shows a sharp decrease and beyond 50 gm significant crack occurs which may be due to the release of internal stress generated locally by indentation [64].

3.2.7. Kurtz-Perry Powder SHG Test. The second-order NLO property of the L-threonine crystal was tested using KurtzPerry powder SHG measurement and its relative conversion efficiency has been found out as described in the introduction section. An Nd : YAG laser with modulated radiation of $1064 \mathrm{~nm}$ was used as the optical source and directed
TABLE 5: Comparison of SHG efficiencies of different organic NLO crystals relative to KDP equaling 1.0.

\begin{tabular}{ll}
\hline L-threonine & 1.15 \\
L-alanine & 0.20 \\
L-proline & 0.20 \\
L-asparagine & 0.25 \\
L-Leucine & 0.19 \\
\hline
\end{tabular}

on the powdered sample through a visible blocking filter. The doubling of frequency was confirmed by the emission of green radiation of wavelength $532 \mathrm{~nm}$ collected by a monochromator after separating the $1064 \mathrm{~nm}$ pump beam with an IR-blocking filter. The SHG efficiency of L-threonine was found to be higher than that of many organic NLO crystals and the values were compared in Table 5.

\subsection{Influence of Isoelectric $\mathrm{pH}$ on the Crystalline \\ Properties of L-Threonine}

3.3.1. Optical Transmittance Studies. UV-Vis-NIR spectrum of L-threonine crystals (grown at different $\mathrm{pH}$ ) in the range 190 and $1500 \mathrm{~nm}$ is represented in Figure 26. The optical transparency region lies between 190 and $1100 \mathrm{~nm}$. Cutoff wavelength $\lambda_{\text {cutoff }}$ varies with $\mathrm{pH}$. Lower UV cutoff of Lthreonine crystal grown at isoelectric point (PI) occurs at $198 \mathrm{~nm}$ whereas for a crystal at $\mathrm{pH}=4.4$ and $\mathrm{pH}=6.7$, $\lambda_{\text {cutoff }}$ falls at 206 and $208 \mathrm{~nm}$. Maximum absorbance $\left(\lambda_{\max }\right)$ of L-threonine crystal grown at PI shifts towards the shorter wavelength side thereby increasing the width of transparency window of the title compound [65]. This favours further for 


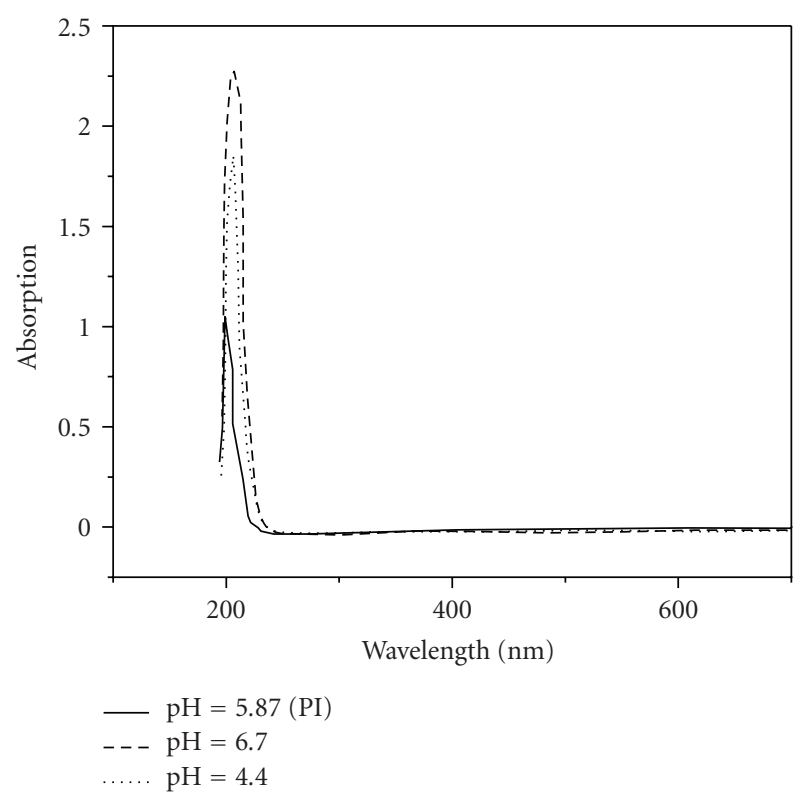

Figure 26: UV-Vis-NIR spectrum of L-threonine at different $\mathrm{pH}$ values.

the material to act as a potential candidate for NLO device applications even in the UV region.

3.3.2. Effect of Isoelectric pH on Powder SHG Efficiency. KurtzPerry powder SHG efficiencies were measured for the polycrystalline samples of various $\mathrm{pH}$ values. The average grain sizes of all samples were found to be same. The powder SHG efficiency of L-threonine sample at isoelectric $\mathrm{pH}$ was found to be higher than the other two-polycrystalline samples. The powder SHG efficiency of L-threonine polycrystalline sample at the isoelectric $\mathrm{pH}$ was found to be 1.2 times that of KDP, and the samples grown at other $\mathrm{pH} 4.40$ and 6.70 have 0.70 and 0.5 times relative to KDP. This is shown in Figure 27.

3.3.3. Effect of $\mathrm{pH}$ on Crystal Quality. In isoelectric $\mathrm{pH}$, the net charge of the dipolar zwitterionic species of $\mathrm{L}$ threonine $\mathrm{CH}_{3} \mathrm{CH}(\mathrm{OH}) \mathrm{CHNH}_{3}{ }^{+} \mathrm{COO}^{-}$is zero, whereas in the solution containing $\mathrm{pH}>\mathrm{PI}$ and $\mathrm{pH}<\mathrm{PI}$ possess two possible ionized species $\mathrm{CH}_{3} \mathrm{CH}(\mathrm{OH}) \mathrm{CHNH}_{3}{ }^{+} \mathrm{COOH}$ and $\mathrm{CH}_{3} \mathrm{CH}(\mathrm{OH}) \mathrm{CHNH}_{2} \mathrm{COO}^{-}$. In the former case, that is, $\mathrm{pH}>\mathrm{PI}$, the solution contains excess positive charge, and in latter, the solution has net negative charge. Crystals grown at $\mathrm{pH}=4.40$ and $\mathrm{pH}=6.70$ show poor optical quality. This is due to the fact that the net positive and negative charge tends to behave as impurities in the crystal lattice, which might be the predominant cause for the crystal opacity and optical deterioration of those crystals. But for a crystal grown at isoelectric conditions, better optical quality crystals have been harvested since the solution strongly limits the contaminations from the ionic species itself [65]. The crystalline quality of L-threonine varies with $\mathrm{pH}$ and it is further confirmed by High-resolution X-ray diffraction analysis (HRXRD).

3.3.4. Multicrystal X-Ray Diffractometer. In order to test the crystalline quality of the grown samples at different $\mathrm{pH}$,

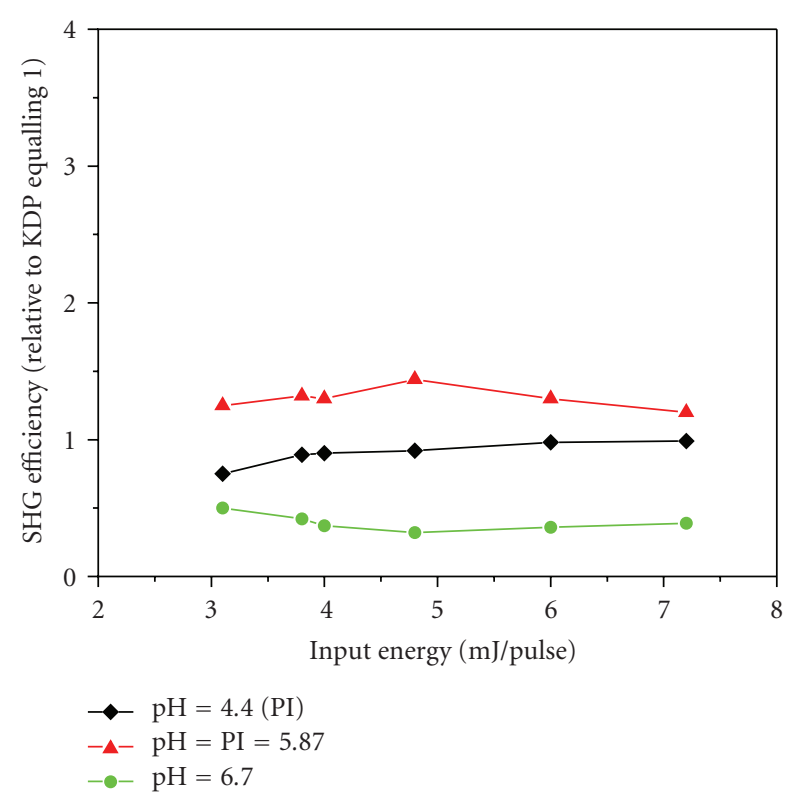

FIGURE 27: Powder SHG efficiencies of L-threonine at different $\mathrm{pH}$ values.

the crystals have been subjected to High-resolution X-ray diffraction analysis [66]. A multicrystal X-ray diffractometer (MCD), developed at National Physical Laboratory (NPL), New Delhi [67], has been used to record high-resolution diffraction curves (DCs). In this system a fine focus $(0.4 \times$ $8 \mathrm{~mm}$; $2 \mathrm{~kW}$ Mo) X-ray source energized by a well-stabilized Philips X-ray generator (PW 1743) was employed. The well-collimated and monochromated $\mathrm{MoK} \alpha_{1}$ beam obtained from the three monochromator Si crystals set in dispersive $(+,-,-)$ configuration has been used as the exploring $\mathrm{X}$ ray beam. This arrangement improves the spectral purity $\left(\Delta \lambda / \lambda \ll 10^{-5}\right)$ of the $\operatorname{MoK} \alpha_{1}$ beam. The divergence of the exploring beam in the horizontal plane (plane of diffraction) was estimated to be $\ll 3$ arc sec. The specimen crystal is aligned in the $(+,-,-,+)$ configuration. Due to dispersive configuration, though the lattice constants of the monochromator crystal(s) and the specimen are different, the unwanted dispersion broadening in the diffraction curve of the specimen crystal is insignificant. The specimen can be rotated about a vertical axis, which is perpendicular to the plane of diffraction, with minimum angular interval of 0.5 arc sec. The diffracted intensity is measured by using a scintillation counter and is mounted with its axis along a radial arm of the turntable. The rocking or diffraction curves were recorded by changing the glancing angle (angle between the incident X-ray beam and the surface of the specimen) around the Bragg diffraction peak position $\theta_{B}$ starting from a suitable arbitrary glancing angle (denoted as zero). The detector was kept at the same angular position $2 \theta_{B}$ with wide opening for its slit, the so-called $\omega$ scan.

3.3.5. HRXRD Analysis. Before going to record the diffraction curve, the specimen surface was prepared by lapping and polishing and then chemically etched by a nonpreferential chemical etchent mixed with water and acetone in $1: 2$ ratio. 


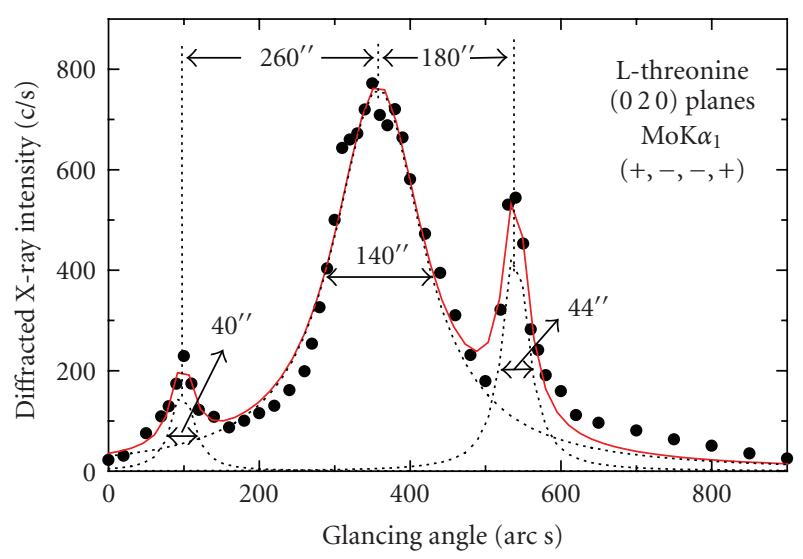

FIGURE 28: HRXRD curve recorded for L-threonine single crystal grown at $\mathrm{pH}=6.70$ for $\left(\begin{array}{lll}0 & 2 & 0\end{array}\right)$ diffracting planes.

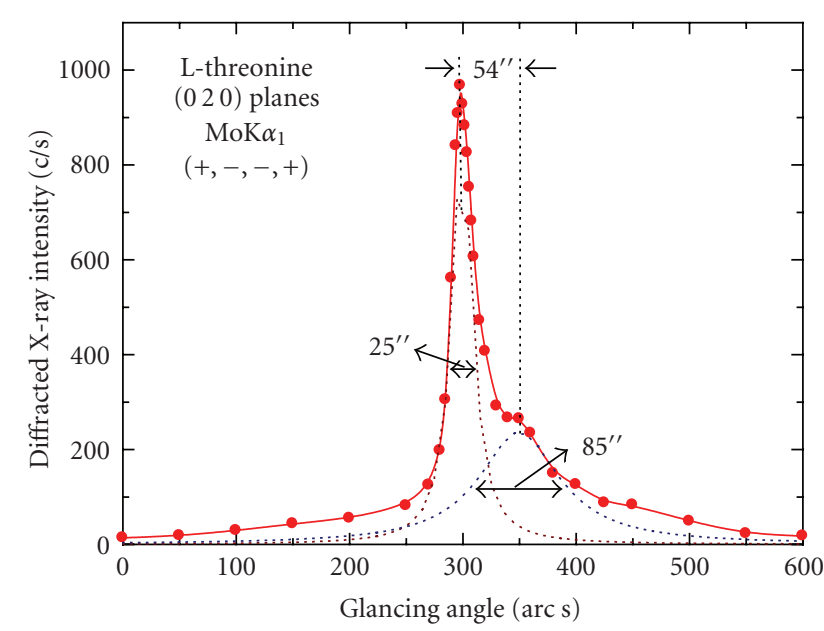

FIGURE 29: HRXRD curve recorded for L-threonine single crystal grown at isoelectric $\mathrm{pH}$ for $\left(\begin{array}{lll}0 & 2 & 0\end{array}\right)$ diffracting planes.

Figures 28 and 29 show the high-resolution diffraction curves (DCs) recorded for a typical L-threonine single crystals grown at different $\mathrm{pH}$ using $\left(\begin{array}{lll}0 & 2 & 0\end{array}\right)$ diffracting planes in symmetrical Bragg geometry by employing the multicrystal $\mathrm{X}$-ray diffractometer described above with $\mathrm{MoK} \alpha_{1}$ radiation. For a crystal grown at $\mathrm{pH}$ 6.70, the solid line (convoluted curve) in Figure 18 is well fitted with the experimental points represented by the filled circles.

On deconvolution of the diffraction curve, it is clear that the curve contains two additional peaks, which are 260 and 180 arc sec away from the main peak. These two additional peaks correspond to two internal structural low-angle (tilt angle $\geq 1$ arc min but less than a degree) boundaries [68], whose tilt angles (misorientation angle between the two crystalline regions on both sides of the structural grain boundary) are 260 and 180 arc sec from their adjoining regions. The FWHM (full width at half maximum) of the main peak and the low-angle boundaries is, respectively, 140, 40 , and $44 \mathrm{arcsec}$. Though the specimen contains very lowangle boundaries, the relatively low angular spread of around
800 arc sec of the diffraction curve and the low FWHM values show that the crystalline perfection is reasonably good.

But for a crystal grown at isoelectric $\mathrm{pH}$, though the curve (Figure 29) does not contain a single diffraction peak, the tilt angle of the additional satellite peak observed nearby to the main peak is only 54 arc sec from its adjoining region. This is far better from the earlier sample. Moreover the FWHM values of the main peak and the very lowangle boundary are 25 and 85 arc sec, respectively. It is worthwhile to note here that Vijayan et al. [69] have recently reported HRXRD analysis on L-alanine single crystals at various positions of a single crystal. The FWHM value of the main peak for an L-alanine seed crystal was reported as 26 arc sec, whereas in the present case, even for a bulk single crystal grown at isoelectric $\mathrm{pH}$, the FWHM of the main peak is only $25 \mathrm{arcsec}$. Though the specimen contains a lowangle boundary, the relatively low angular spread of around $300 \mathrm{arc} \mathrm{sec}$ of the diffraction curve and the low FWHM values show that the crystalline perfection of the specimen grown at isoelectric $\mathrm{pH}$ is much better than that of the crystal grown at $\mathrm{pH}=6.70[70]$. The segregation of impurities during the growth process could be responsible for the observed very low-angle boundary. It may be mentioned here that such a very low-angle boundary could be detected with well-resolved peak in the diffraction curve only because of the high-resolution of the multicrystal X-ray diffractometer used in the present studies. The influence of such minute defects on the NLO properties is very insignificant. However, a quantitative analysis of such unavoidable defects is of great importance, particularly in case of phase-matching applications.

3.4. Deuteration Effects on L-Threonine Single Crystals. Deuteration of many NLO crystals yielded improved optical properties. In fact deuterated KDP is still considered as one of the best electro-optic modulators with large electro-optic coefficient [71]. Deuteration on zinc tris thiourea sulphate (ZTS) [72] and L-arginine phosphate (LAP) [73] showed that electro-optic properties have been improved greatly. The reduction in optical absorption and the increase in transparency window have also been observed for deuterated LAP [74]. Following from the above ideas, an attempt has been made to grow the deuterated crystals of L-threonine.

3.4.1. Powder X-Ray Diffraction Analysis. To test the crystallinity of the samples, powder X-ray diffraction patterns were obtained at room temperature using a Rigaku D/max-A diffractometer fitted with $\mathrm{CuK} \alpha$ radiation $(\lambda=1.54598 \AA)$ for both the deuterated and undeuterated L-threonine polycrystalline samples. All of the peaks observed for both deuterated and normal L-threonine were at identical positions.

The estimated lattice parameters $a=13.611 \AA, b=$ $7.738 \AA$, and $c=5.144 \AA$ show that there is no change in the crystal structure of L-threonine on deuteration. But the intensities of the many diffracted peaks were found to be increased on deuterating the compound. The decrease in the full-width half-maximum (FWHM) values of all peaks and the increased intensities confirm that crystalline quality 


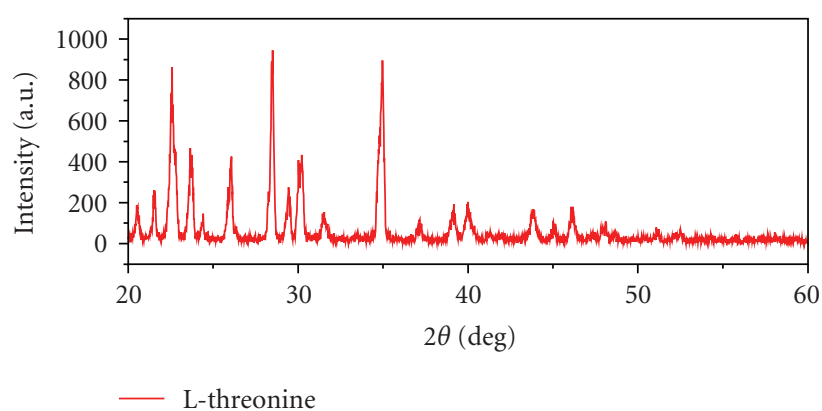

(a)

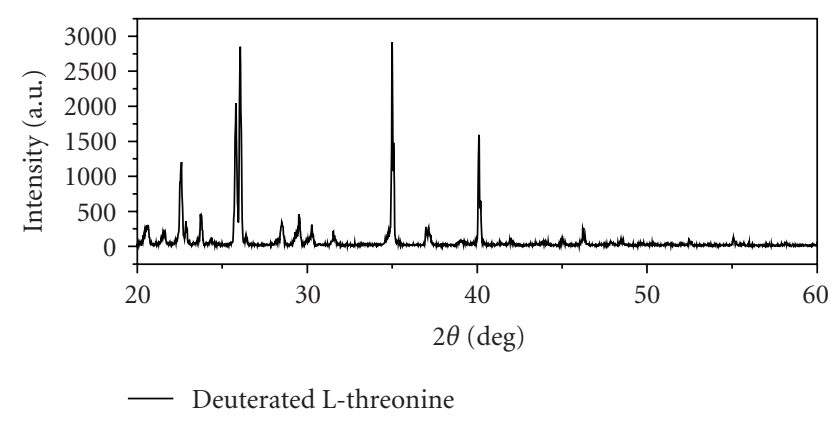

(b)

FIgURe 30: Powder X-ray diffraction of L-threonine and deuterated L-threonine.

TABle 6: $2 \theta$ and $(h k l)$ values of L-threonine and its deuterated analog.

\begin{tabular}{llll}
\hline $2 \theta$ & $h$ & $k$ & $l$ \\
\hline 20.58 & 0 & 1 & 1 \\
21.58 & 2 & 0 & 1 \\
22.58 & 3 & 1 & 0 \\
22.86 & 0 & 2 & 0 \\
24.36 & 2 & 1 & 1 \\
26.06 & 3 & 0 & 1 \\
26.34 & 2 & 2 & 0 \\
28.46 & 4 & 1 & 0 \\
29.47 & 1 & 2 & 1 \\
30.38 & 3 & 2 & 0 \\
35.00 & 3 & 2 & 1 \\
37.10 & 2 & 3 & 0 \\
39.16 & 0 & 3 & 1 \\
40.10 & 1 & 3 & 1 \\
42.04 & 1 & 2 & 2 \\
44.04 & 4 & 0 & 2 \\
45.08 & 6 & 1 & 1 \\
46.22 & 6 & 2 & 0 \\
55.02 & 1 & 1 & 3 \\
\hline
\end{tabular}

of the sample has been much improved on deuteration (Figure 30). Two thetas and their corresponding Miller planes are listed in Table 6.

3.4.2. Fourier Transform Infrared Spectroscopy (FTIR). FTIR spectra of deuterated and normal L-threonine were recorded using a BRUKER IFS 66 FTIR spectrophotometer in the range $400-4000 \mathrm{~cm}^{-1}$ by $\mathrm{KBr}$ pellet technique. The deuterated spectrum shown at Figure 31 at different recrystallization stages shows that degree of deuteration increases on multiple recrystallization with $\mathrm{D}_{2} \mathrm{O}$.

In the figure, the spectra DLT-1 and DLT-2 represent the deuterated salts of L-threonine after first and second recrystallizations. Further recrystallization yields a similar spectra showing that the $\mathrm{NH}$ and $\mathrm{OH}$ site protons are completely deuterated. The peak positions and their assignments are listed in Table 7. The medium peaks at 550 and
$667 \mathrm{~cm}^{-1}$ in DLT-2 are assigned to out-of-plane bending vibrations of $\mathrm{O}-\mathrm{D}$. These peaks were not observed in DLT1 and normal L-threonine. The peak at $933 \mathrm{~cm}^{-1}$ represents rocking vibrations of $\mathrm{ND}_{3}{ }^{+}$. The strong peaks at 1102,1157 , and $1182 \mathrm{~cm}^{-1}$ correspond to symmetric and degenerate deformation vibrations of $\mathrm{ND}_{3}{ }^{+}$. These peaks were also observed in DLT-1 but with the less intensity.

The characteristic peaks observed at 2341 and $2250 \mathrm{~cm}^{-1}$ are due to the stretching vibrations of $\mathrm{O}-\mathrm{D}$. These values are in good agreement with the earlier report [75]. A shallow peak at $2158 \mathrm{~cm}^{-1}$ is due to the stretching of $\mathrm{ND}^{+}$. In DLT1 , the stretching vibrations of $\mathrm{O}-\mathrm{D}$ were shifted towards the high-frequency regions at 2349 and $2223 \mathrm{~cm}^{-1}$. The characteristic vibrations of carboxyl group $\mathrm{COO}^{-}$(rock) and $\mathrm{COO}^{-}$(wagging) produce peaks at 560 and $707 \mathrm{~cm}^{-1}$. These peaks have been shifted to 535 and $701 \mathrm{~cm}^{-1}$ for deuterated L-threonine [76]. This is in accordance with the earlier work cited in [77].

3.4.3. Thermal Analysis. The thermal strength of the normal and deuterated crystals has been determined by differential scanning calorimetry (DSC) using NETZSCH-Geratebau $\mathrm{GmbH}$ thermal analyzer in the temperature range $30-400^{\circ} \mathrm{C}$ in nitrogen atmosphere. Same heating rate of $5^{\circ} \mathrm{C} /$ minute was applied to both of the samples. A single endothermic peak is observed at $268.1^{\circ} \mathrm{C}$ for deuterated L-threonine, which actually gives the decomposition temperature of the grown crystal (Figure 32). This is slightly higher than that of pure L-threonine crystals, which decomposes at $262^{\circ} \mathrm{C}$.

3.4.4. Dielectric Measurements. Dielectric studies were performed on deuterated L-threonine single crystal at room temperature in the frequency range $1 \mathrm{KHz}-1 \mathrm{MHz}$. The sample has been coated with conductive silver paint for metallic contacts. A sinusoidal a.c. voltage was applied to the sample through the silver electrodes for various frequencies. Capacitance developed by the crystal was recorded and the dielectric constant has been calculated using the area and thickness of the sample.

At lower frequencies, dielectric constant initially increases due to the space-charge polarization of molecular dipoles. But for higher frequencies, it is almost constant up to the maximum frequency studied. The dielectric constant 


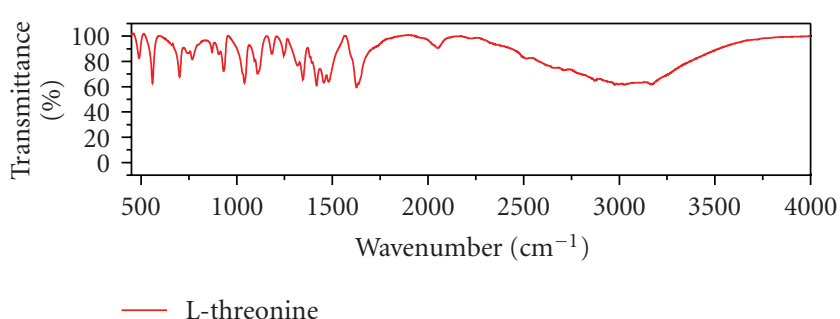

(a)

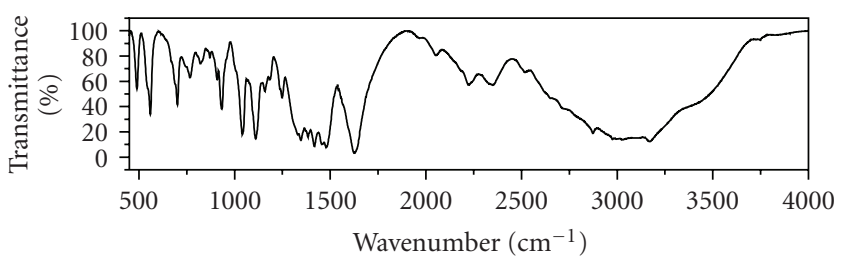

DLT-1

(b)

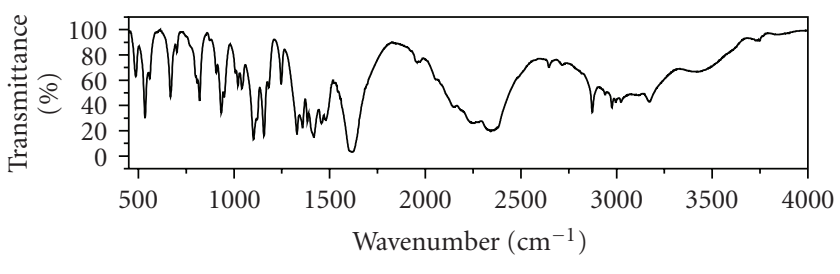

D-LT2

(c)

FIGURE 31: FTIR spectra of L-threonine and deuterated L-threonine crystals.

TABLE 7: Deuterated and undeuterated L-threonine assignments on IR bands.

\begin{tabular}{|c|c|c|}
\hline \multicolumn{2}{|c|}{ Wavenumber $\left(\mathrm{cm}^{-1}\right)$} & \multirow[b]{2}{*}{ Assignments } \\
\hline Undeuterated & Deuterated & \\
\hline 3157,3021 & & $\mathrm{~N}-\mathrm{H}$ stretch \\
\hline \multirow[t]{4}{*}{2975} & 2975 & $\mathrm{C}-\mathrm{H}$ stretching \\
\hline & 2341,2873 & Symmetric stretching of $\mathrm{CH}_{3}{ }^{+}$ \\
\hline & 2250 & Symmetric stretching of O-D \\
\hline & 2158 & Stretching of $\mathrm{ND}_{3}{ }^{+}$ \\
\hline 2050 & & Degenerative deformation of $\mathrm{NH}_{3}{ }^{+}$and torsional vibration of $\mathrm{NH}_{3}{ }^{+}$ \\
\hline 1623 & & Asymmetric stretching of $\mathrm{COO}^{-}$ \\
\hline \multirow[t]{2}{*}{1481} & & Symmetric bending of $\mathrm{NH}_{3}^{+}$ \\
\hline & 1616 & Asymmetric stretching \\
\hline \multirow[t]{2}{*}{1455} & & Degenerative deformation of $\mathrm{CH}_{3}$ \\
\hline & 1457 & Degenerative deformation of $\mathrm{CH}_{3}$ \\
\hline \multirow[t]{4}{*}{1417} & & Symmetric stretching of $\mathrm{CO}_{2}^{-}$ \\
\hline & 1417 & Symmetric stretching of $\mathrm{COO}^{-}$ \\
\hline & 1383 & Symmetric deformation of $\mathrm{CH}_{3}$ \\
\hline & 1358 & $\mathrm{C}-\mathrm{O}$ stretching \\
\hline $1246,1318,1346$ & 1329 & In-plane deformation of $\mathrm{C}-\mathrm{C}-\mathrm{H}$ \\
\hline \multirow[t]{2}{*}{1184} & & $\mathrm{NH}_{3}{ }^{+}$rocking \\
\hline & $1102,1157,1182$ & Degenerate deformation of $\mathrm{ND}_{3}{ }^{+}$ \\
\hline \multirow[t]{2}{*}{1109} & & Rocking vibration of $\mathrm{CH}_{3}$ \\
\hline & 1048,1246 & Rocking of $\mathrm{CH}_{3}$ group \\
\hline \multirow[t]{2}{*}{931} & & $\mathrm{C}-\mathrm{C}$ stretching \\
\hline & 933 & $\mathrm{ND}_{3}{ }^{+}$rocking \\
\hline \multirow[t]{2}{*}{767} & & Out-of-plane bending vibration of $\mathrm{CO}_{2}^{-}$ \\
\hline & 711 & Wagging of $\mathrm{CO}_{2}^{-}$ \\
\hline \multirow[t]{3}{*}{701} & & In-plane deformation of $\mathrm{COO}^{-}$ \\
\hline & 701 & Wagging of $\mathrm{CO}_{2}^{-}$ \\
\hline & 550,667 & Out-of-plane bending vibration of OD \\
\hline 489 & 535 & $\mathrm{CO}_{2}^{-}$rocking \\
\hline
\end{tabular}




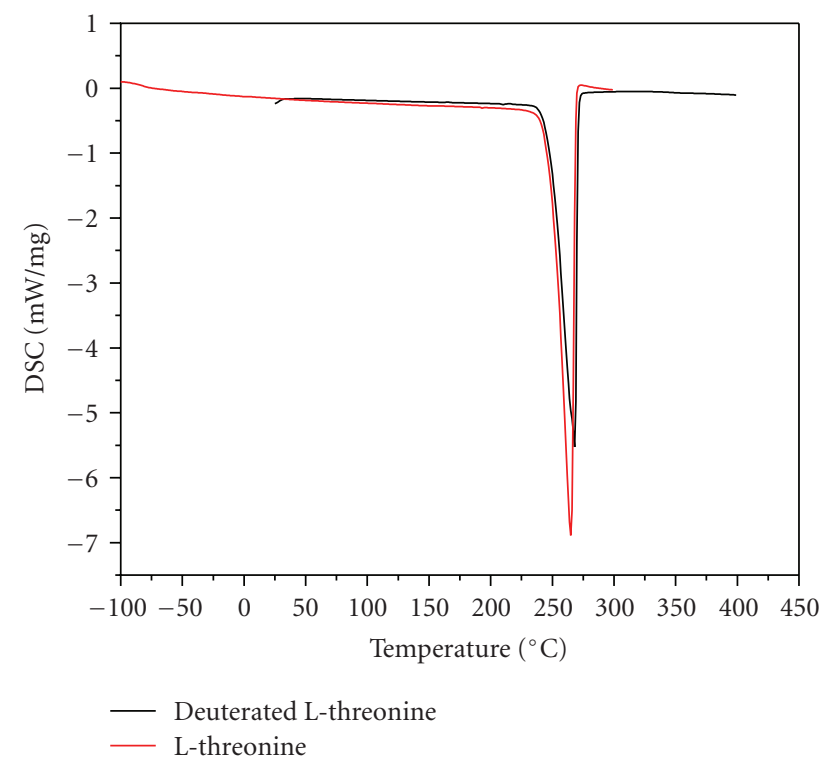

Figure 32: DSC curve of L-threonine and deuterated L-threonine single crystals.

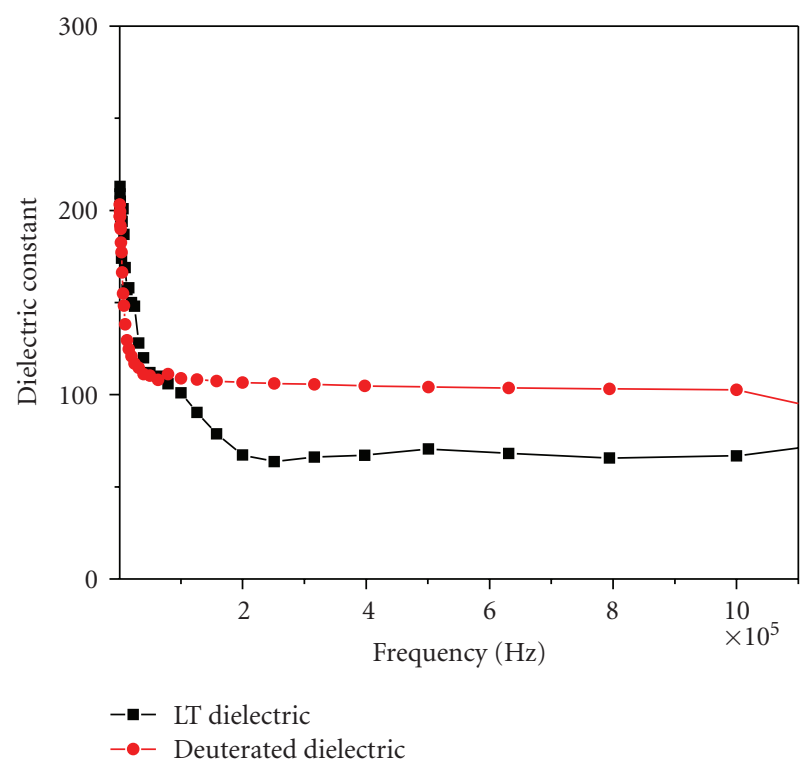

Figure 33: Dielectric constant of pure and deuterated L-threonine single crystal at various frequencies.

of deuterated L-threonine was found to be 102 at $1 \mathrm{MHz}$ whereas the undeuterated crystal shows 66 at the same frequency. Dielectric loss calculated at various frequencies reveals that power loss of the sample on applying electrical energy was found to be negligible. The dielectric constant and dielectric loss at various frequencies are shown in Figures 33 and 34, respectively. The dielectric constant of deuterated L-threonine is greater than that of commonly used electrooptic (EO) modulators like potassium di-deuterated phosphate (D-KDP) and potassium di-deuterated arsenate (D-KDA) [78, 79]. Since the electro-optic coefficient is directly proportional to the dielectric constant of the material [80], the increase in the dielectric constant of deuterated

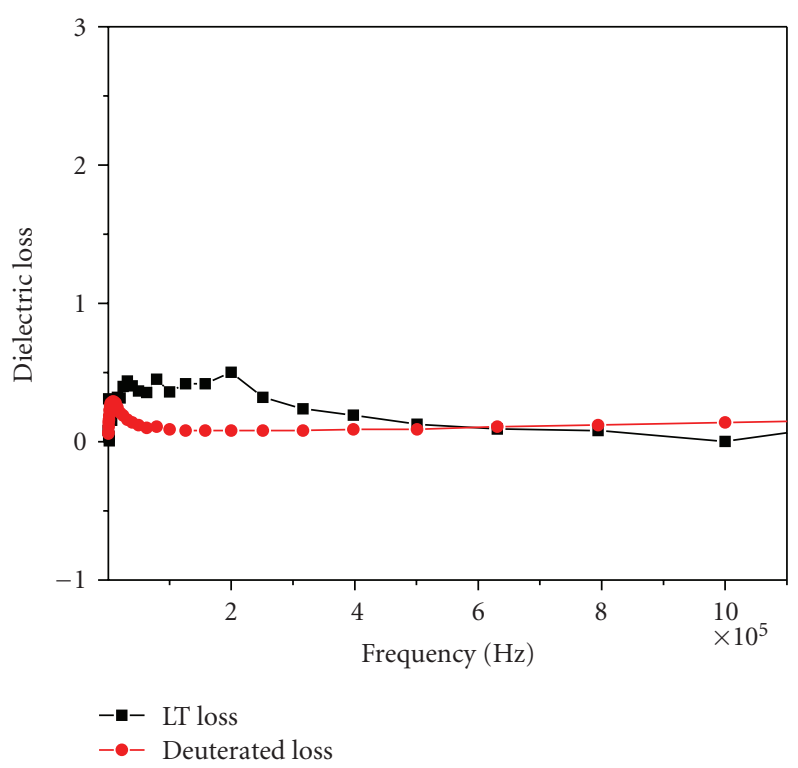

Figure 34: Dielectric loss of pure and deuterated L-threonine single crystal at various frequencies.

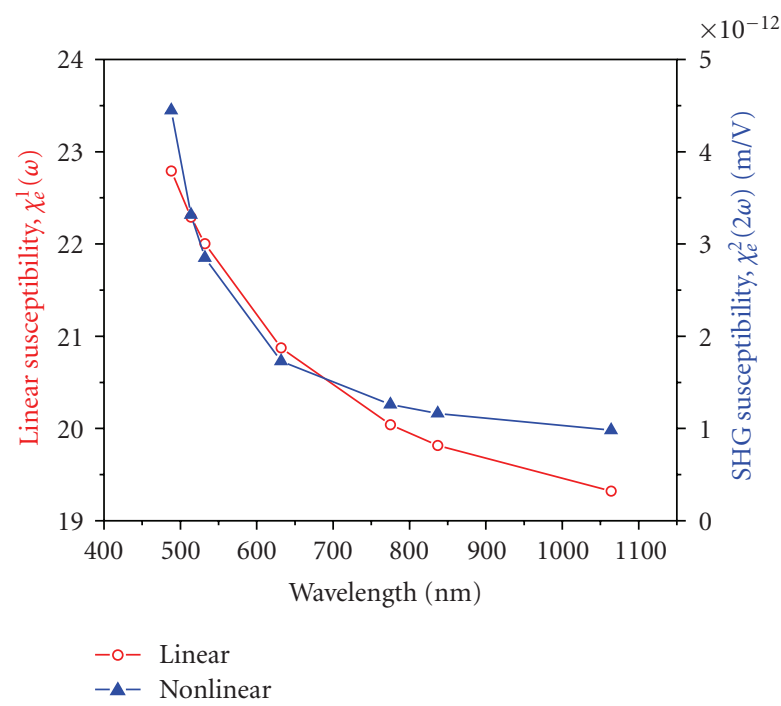

FIGURE 35: Dispersion of Linear and Nonlinear (SHG) susceptibility with wavelength for L-threonine single crystals.

L-threonine could enhance its electro-optic coefficient and further should have a lower half-wave retardation voltage [76].

3.4.5. Optical Transmittance Studies. UV-Vis-NIR spectrum has been recorded for both deuterated and normal Lthreonine single crystals using a Varian cary 5E UV-Vis-NIR spectrophotometer in the wavelength range $200-1200 \mathrm{~nm}$. The lower UV cutoff wavelength of both deuterated and undeuterated samples remains invariant. The absorption range in the whole spectral range studied is found to be identical for both of the compounds. Hence the deuteration has not altered the transmission range of the compound. 
3.4.6. Kurtz-Perry Powder SHG Test. Kurtz-Perry powder SHG efficiency of the crystal has been measured for the deuterated compound. The average grain sizes were found to be similar in both pure and deuterated L-threonine polycrystalline samples. The output intensity of the deuterated crystal was found to be 0.91 times than that of KDP. The decrease in the SHG output intensity for the title crystal when compared to normal L-threonine polycrystalline samples (1.2 KDP) may be due to the exchange of deuterium in $\mathrm{NH}$ and $\mathrm{OH}$ sites that causes less perturbation on the incident highpower laser beam. The shift in the stretching frequencies of $\mathrm{N}-\mathrm{D} \cdots \mathrm{O}$ and $\mathrm{O}-\mathrm{D} \cdots \mathrm{O}$ as well as the carboxylate groups towards the lower-energy side also accounts for the decrease in the second-order nonlinear polarizability [81] of the deuterated crystal.

\subsection{Application and Calculation of Second-Order NLO \\ Parameters for L-Threonine Single Crystals}

3.5.1. Second Harmonic Generation (SHG). In literature, no reports are available on electro-optic and SHG susceptibility measurements on L-threonine, so an attempt in evaluating the electro-optic and second-order nonlinear parameters using three-dimensional anharmonic oscillator model would be very useful for the experimental determination of above optical entities. Since the second harmonic wavelengths of many laser sources like Ar, GaAs, $\mathrm{He}-\mathrm{Ne}$, and $\mathrm{Nd}$ : YAG lie within the transparent range (200-800 nm) of L-threonine, various NLO coefficients have been determined for different wavelengths within 488-1064 nm.

In order to determine the electro-optic and SHG susceptibility, electronic, and ionic contributions to the linear susceptibility have to be found out. They are expressed as

$$
\begin{aligned}
& \chi_{e}^{(1)}(\omega)=\frac{N_{e}^{2} e_{e}^{2}}{\left[m_{e} \varepsilon_{o}\left(\omega_{e}^{2}-\omega^{2}\right)\right]}, \\
& \chi_{i}^{(1)}(\omega)=\frac{N_{i}^{2} e_{i}^{2}}{\left[m_{i} \varepsilon_{o}\left(\omega_{i}^{2}-\omega^{2}\right)\right]} .
\end{aligned}
$$

Parameters required to calculate the linear susceptibility are given below.

\section{Input Data.}

$$
\begin{aligned}
& \text { Electron density } N_{e}{ }^{\mathrm{a}}=4.697 \times 10^{29} / \mathrm{m}^{3}, \\
& \text { Electronic charge } e_{e}=1.602 \times 10^{-19} \mathrm{C}, \\
& \text { Mass of the electron } m_{e}=9.11 \times 10^{-31} \mathrm{Kg}, \\
& \text { Electronic resonant frequency } \omega_{e}{ }^{\mathrm{b}}=8.971 \times 10^{15} \mathrm{~Hz},
\end{aligned}
$$

haveing ${ }^{a}$ computed from the density of L-threonine and ${ }^{\mathrm{b}}$ computed from UV absorption edge of L-threonine.

With the help of the input data, $\chi_{e}^{(1)}(\omega)$ for the energy of photons lying in the optical frequency range has been computed. As the ionic contribution $\chi_{i}(\omega)$ to SHG is almost negligible in comparison to $\chi_{e}^{(1)}(\omega)$ for the frequency range under study, $\chi^{2}(2 \omega, \omega, \omega)$ and $\chi^{2}(\omega, \omega, 0)$ mainly depend on $\chi_{e}^{2}(\omega)$.Therefore, the effects of A, B and C parameters in (19)

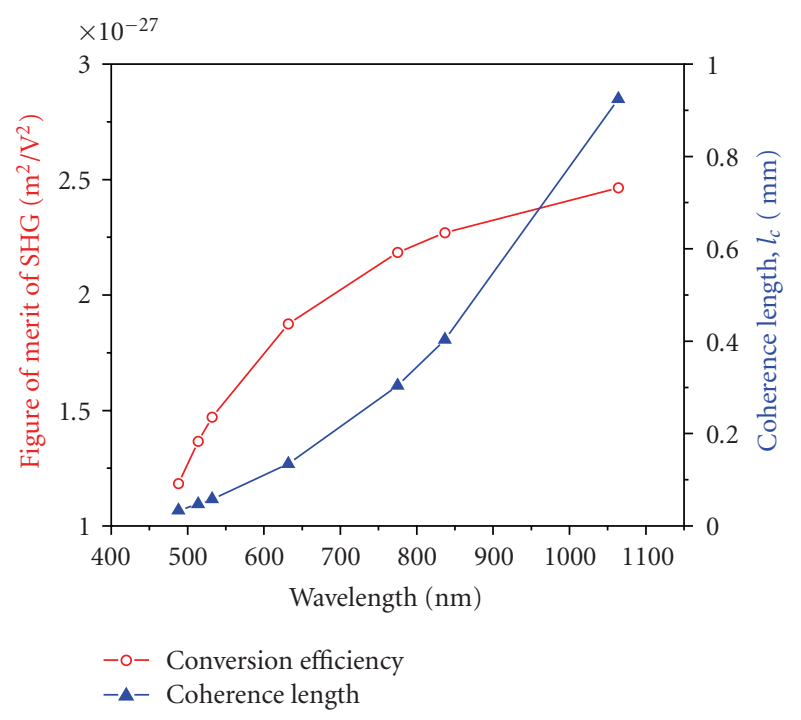

FIGURE 36: SHG conversion efficiency and coherence length for various input wavelengths.

and (20) have been ignored. To calculate the pure electronic contribution to NLO susceptibilities, parameter " $D$ " has been evaluated using the value of $d_{i j}$ coefficient reported by Singh et al. [82] for D-threonine at $1064 \mathrm{~nm}$. The value of $\mathrm{D}$ was found as $9.2062 \times 10^{8} \mathrm{~kg} / \mathrm{m} \cdot \mathrm{sec}^{2}$. For a crystal class of $\mathrm{P} 2{ }_{1} 22_{1}$, the only nonzero elements contributing for SHG are $d_{14}, d_{25}$, and $d_{36}$ [83]. Using Kleinmann conjuncture [84], it can be written as SHG coefficients $d_{14}=d_{25}=d_{36}$, and hence $\chi^{2}(2 \omega, \omega, \omega)$ could be found using the relation [85]

$$
d_{i j}=\frac{\chi_{e}^{(2)}(2 \omega)}{2} \text {. }
$$

Using the parameter D and $\chi_{e}^{(1)}(\omega)$, SHG susceptibilities of L-threonine have been found for various wavelengths from (19). The dispersions of linear and nonlinear (SHG) susceptibility of L-threonine with wavelength are shown in Figure 35. The decrease in the linear and SHG susceptibility with increase in wavelength appears to be similar to that in the study made on CdTe [48].

In the SHG experiment, the second harmonic light moves through the crystal in the same direction of the incident beam but with a slower velocity because of the higher index of refraction at the shorter wavelengths. Eventually, the second harmonic component reaches a point further along in the crystal where new second harmonic light is being generated, which is exactly $180^{\circ}$ out of phase to $\mathrm{SH}$ ray that has generated earlier, thus causing destructive interference and the SHG drops to zero. So, whenever the pathlength equals integral number of interference distances, SHG intensity becomes zero. From the above reason, it is evident that a crystal need not be thicker than one-half of SHG interference distance called "coherence length" which is in the order of microns depending on the wavelength of the fundamental beam. From this model, coherence length associated with the SHG is found out by the expression

$$
l_{c}=\frac{\lambda}{4}[n(\omega)-n(2 \omega)]
$$




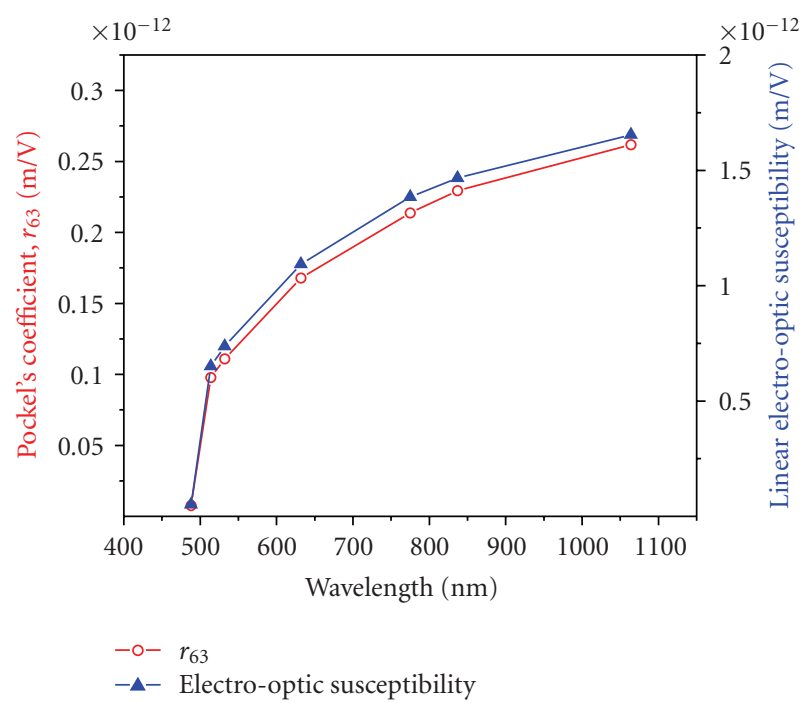

FIGURE 37: Variation of $r_{63}$ and electro-optic susceptibility with wavelength.

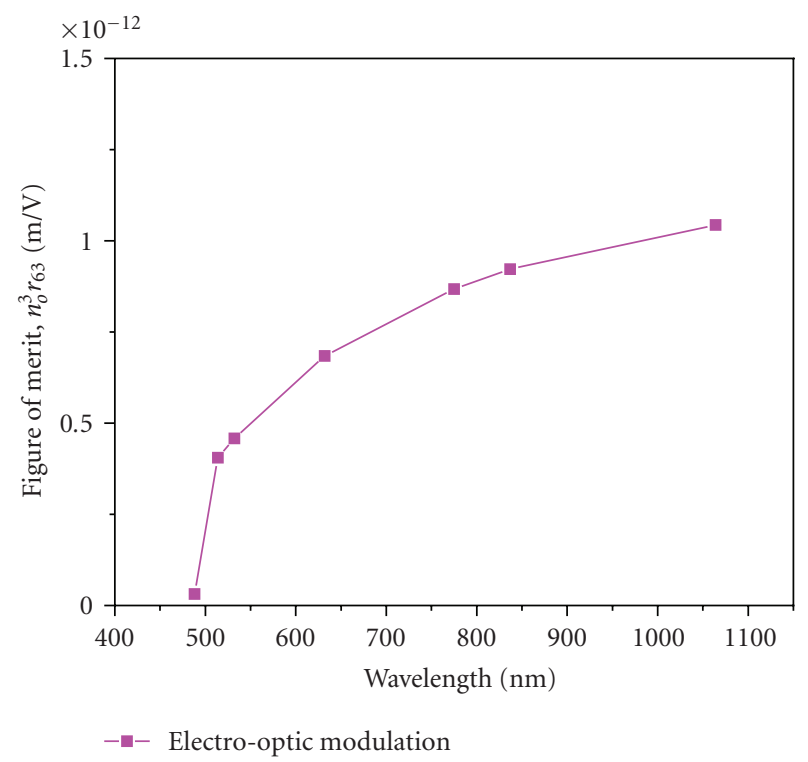

FIGURE 38: Wavelength versus Figure of Merit of EO modulation.

where $\lambda$ is the wavelength of the fundamental wave in $\mathrm{nm}$ and $n^{\omega}, n^{2 \omega}$ are refractive index at fundamental and second harmonic wavelengths. $n^{\omega}$ and $n^{2 \omega}$ values of L-threonine were found out with the help of the following equations.

Since $n^{2}(\omega) \simeq \varepsilon_{r}$ at optical frequencies,

$$
\begin{aligned}
n^{\omega} & =\left[1+\chi_{e}^{(1)}(\omega)\right]^{1 / 2}, \\
n^{2 \omega} & =\left[1+\chi_{e}^{(1)}(2 \omega)\right]^{1 / 2} .
\end{aligned}
$$

It has been found that the coherence lengths calculated from the above equation were in the order of microns. Figure 36 shows that coherence length increases not only with the crystal length but also with the wavelengths

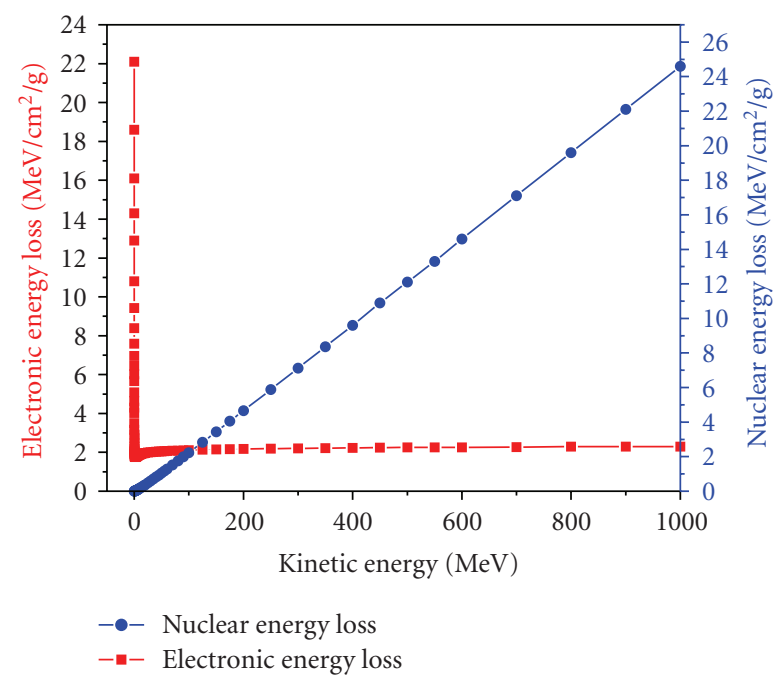

FIGURE 39: Electronic and nuclear energy loss for various incident electron energies in L-threonine single crystals.

used. Coherence length $\left(l_{c}\right)$ of L-threonine crystal was also calculated experimentally [82] and reported as few microns for $1064 \mathrm{~nm}$. The discrepancy between the experimental values and the present model may be due to the fact that we have completely ignored the contribution $\chi_{i}^{(1)}(\omega)$, a complex quantity which in turn affects the refractive index of the crystal.

One more parameter, which characterizes the SHG property of materials, is figure of merit of SHG. This can be found out using the equation

$$
\text { Figure of merit }=\frac{d_{i j}^{2}}{n^{2 \omega}\left[n^{\omega}\right]^{2}} .
$$

This quantity indicates how efficiently the fundamental optical beam is converted to a beam of frequency $2 \omega$. As we have used Kleinmann condition, $d_{i j}$ values was calculated by using (29) for various wavelengths. The dispersion of figure of merit of SHG with wavelength is shown in Figure 36 [86].

3.5.2. Linear Electro-Optic Susceptibilities. Following Sharma et al. [49], electro-optic susceptibility of L-threonine has been found using the expression given in (20). The parameter $\mathrm{D}$ calculated earlier was used here to obtain $\chi_{e}^{2}(\omega, \omega, 0)$. The dispersion of EO susceptibility with wavelength is shown in Figure 37. Similarly electro-optic coefficient $\left(r_{i j}\right)$ was also calculated by the following relation:

$$
r_{i j}=-\frac{\chi_{e}^{2}(\omega,, \omega,, 0)}{n_{o}^{4}} .
$$

Here the negative sign indicates the pure electronic contribution to EO coefficient.

Efficient modulators can be constructed based on the value of Pockel's coefficient $\left(r_{i j}\right)$ usually found either through longitudinal mode or by transverse mode. As $r_{i j}$ is independent of the crystal dimensions in the longitudinal EO 


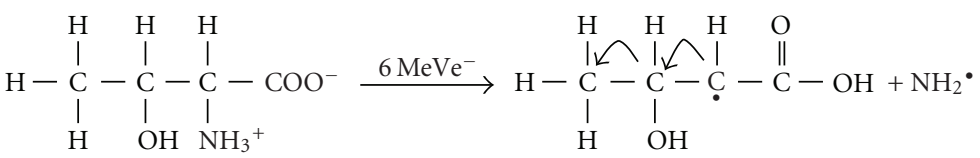

FIgURE 40: Deaminated free radical formation due to electron irradiation in L-threonine.

TABLE 8: Dispersion of second order NLO parameters with wavelength.

\begin{tabular}{lcccccc}
\hline $\begin{array}{l}\text { Wavelength } \\
(\mathrm{nm})\end{array}$ & $\begin{array}{c}\chi_{e}^{2} \\
(\mathrm{pm} / \mathrm{V})\end{array}$ & $\begin{array}{c}\text { Figure of merit of SHG } \\
\left(10^{-27} \mathrm{~m}^{2} / \mathrm{V}^{2}\right)\end{array}$ & $\begin{array}{c}\text { Coherence length } \\
(\mu \mathrm{m})\end{array}$ & $\begin{array}{c}r_{63} \\
(\mathrm{pm} / \mathrm{V})\end{array}$ & $\begin{array}{c}\text { EO susceptibility } \\
(\mathrm{pm} / \mathrm{V})\end{array}$ & $\begin{array}{c}\text { Figure of merit of EO modulation } \\
(\mathrm{pm} / \mathrm{V})\end{array}$ \\
\hline 488 & 4.44 & 1.18 & 0.03 & 0.007 & 0.05 & 0.12 \\
514 & 3.31 & 1.36 & 0.04 & 0.097 & 0.65 & 1.57 \\
532 & 2.85 & 1.47 & 0.05 & 0.111 & 0.73 & 1.78 \\
632 & 1.73 & 1.87 & 0.13 & 0.167 & 1.09 & 3.68 \\
775 & 1.26 & 2.18 & 0.30 & 0.213 & 1.38 & 3.41 \\
837 & 1.16 & 2.26 & 0.40 & 0.229 & 1.46 & 1.64 \\
1064 & 0.98 & 2.46 & 0.92 & 0.261 & 1.65 & 4.15 \\
\hline
\end{tabular}

modulator, electronic part of " $r$ " coefficient was calculated in the above mode. L-threonine is a highly c-axis-oriented transparent crystal having ( $\left.\begin{array}{lll}1 & 2 & 0\end{array}\right)$ as a prominent plane. Applying the electric field along the c-axis and propagating the beam in the same direction actually singles out $r_{63}$ element of the (lll $\left.\begin{array}{lll}2 & 2 & 2\end{array}\right)$ EO tensor. As the Kleinmann symmetry unifies all possible nonvanishing elements of $\mathrm{P} 2_{1} 2_{1} 2_{1} \quad\left(r_{41}=r_{52}=r_{63}\right)$ space group, generality is not lost in finding the $r_{63}$ element alone. The ordinary index of refraction $\left(n_{0}\right)$ of L-threonine along $z$-axis (crystallographic $c$-axis) for various wavelengths has been taken from [28]. The figure of merit of EO modulation $n_{o}^{3} r_{63}$ has also been determined for L-threonine crystal. Here it has been assumed as $n_{o} \simeq n_{e}=n$ [87].

Variation of figure of merit of EO modulation with wavelength is presented in Figure 38. In all of the cases, linear EO susceptibility, figure of merit of EO modulation, and the electro-optic coefficient were found to be increasing with the decrease in the frequency. This is in contradiction to the SHG susceptibility where there is a linear relationship. Usually Pockel's effect may also be observed at relatively low frequencies (up to gigahertz) so that slower nonlinear polarization mechanism such as ionic polarization can effectively contribute to the " $r$ " coefficient.

Since we have found out the anharmonic potential coefficient "D" alone, $r_{63}$ values presented in Figure 37 are purely electronic in nature. $r_{\text {ionic }}$ could be calculated using nonlinear strength factors A, B \& C in (20). Transverse optic frequency $\left(\omega_{i}\right)$ of L-threonine in (28) may be computed from its optical absorption spectrum in the gigahertz range. So, effective $\mathrm{EO}$ coefficient is given by

$$
r_{\text {eff }}=r_{\text {electronic }}+r_{\text {ionic }}
$$

Just as linear polarizations are frequency dependent, so are the nonlinear polarizabilities. Thus " $r$ " co-efficient mainly characterizes the low-frequency electro-optic nonlinearity and the " $d$ " co-efficient characterizes the optical frequency electronic nonlinearity.
However it is most surprising that most organic materials with almost exclusively electronic non-linear optical polarization have similar efficiencies for SHG as well as for linear EO effect [88]. From this model electro-optic coefficient of L-threonine was found to be $0.05-0.25 \mathrm{pm} / \mathrm{v}$ for the wavelength range under study [83] and the values are comparable to the experimental $r_{63}$ value $0.2-0.3 \mathrm{pm} / \mathrm{v}$ at $633 \& 532 \mathrm{~nm}$ determined for L-alanine amino acid single crystals [63]. The various second-order NLO parameters for different wavelengths have been tabulated in Table 8 .

3.5.3. Miller Delta. Miller delta $[\Delta(\omega)]$ is the empirical relation between the linear and nonlinear susceptibilities.

$$
\begin{aligned}
& \Delta(\omega)=\frac{\chi^{2}(2 \omega)}{\left[\chi^{1}(\omega)^{2} \chi^{1}(2 \omega)\right]} \quad(\text { SHG Susceptibility), } \\
& \Delta(\omega)=\frac{\chi^{2}(\omega)}{\left[\chi^{1}(\omega)^{2} \chi^{1}(0)\right]} \quad \text { (EO Susceptibility). }
\end{aligned}
$$

The significance of this relation is that the quantity is constant to within a factor of 4 for the materials investigated by Miller [89]. The importance of this quantity is that it may sometimes be used to estimate roughly the non-linear susceptibility of unknown effect in a particular medium. It was observed that both classical and quantum mechanical arguments lead to same observed order of magnitude of this quantity [90]. For the present study, Miller delta was calculated for the above two nonlinear effects. The values for both SHG and EO susceptibilities were found to be within the factor of 4 .

3.6. Electron Irradiation Effects on L-Threonine Single Crystals. Amino acid crystals constitute important model systems for the physical properties of more complex macromolecules such as proteins, metalloproteins, and nucleic acids. Besides this biological significance, one of the compounds L-alanine a neutral amino acid has proven to be a good candidate 
for irradiation-dosimetric applications [91, 92]. Electron irradiation-induced defects on amino acids show that there is no substantial decay of free radicals over a period of many years at room temperature [93].

The chemical reaction initiated by the interaction between an energetic ion or particle and a solid matter may be divided into three main groups according to the following physical process.

(i) Reactions between a projectile and target molecules giving the products, which incorporate the projectile (mass deposit effects). Normally when the projectile slows down to several $\mathrm{eV}$, they will react with the target molecules [94].

(ii) Reactions induced by the energy deposition electronically in a solid during the slowing down of the projectile giving products, which do not contain the projectile (energy deposit effects) [95].

(iii) Reaction induced by elastic nuclear collisions or momentum transfers during the slowing down of the projectile giving products, which do not contain the projectile (momentum transfer effects) [96]. In particle irradiation effects using electrons, protons, and neutrons, mostly, energy deposition and momentum transfer occurs very often, as their penetrating power is very high.

Irradiation of materials with high-energy electron bombardment is a proven method of production of Frenkel defects (vacancies and interstitials) without displacement cascades [97]. Controlled production of point defects in materials has been one of the earlier applications of energetic electron beams in the field of radiation damage. It has also been observed that electron irradiation is better than other methods of production of fast switching diodes through manipulative control of defect properties [98]. High-energy electrons can cause atoms to be displaced from normal lattice positions by the transfer of momentum [99]. Recently Ishwar Bhat et al. $[100,101]$ have reported electron irradiation effects on nonlinear optical barium strontium borate single crystals. They have observed the changes in the refractive index and dielectric constant of the crystal.

Presently, electron irradiation effects on L-threonine single crystal have been studied. The irradiation-induced defects can be quantified with the help of tools like electron paramagnetic resonance (EPR), micro-Raman spectroscopy, thermomechanical (TMA) and thermal analyses. The surface defects were also studied by atomic force microscopy (AFM). Post-irradiation measurements like UV-Vis-NIR, Dielectric and powder SHG have also been performed for the samples.

When electrons of definite energy pass through a solid, their energy is decreased. This energy loss is due to the inelastic collisions of the electrons with atomic electrons by which the atoms of the solid get excited or ionized and due to the emission of bremsstrahlung and this leads to deposition of electronic energy to the target matter. There is also a possibility for the incident electrons to pass through the field of atomic nuclei. Since the mass of the nucleus is very much greater than that of the electron, the collision of electrons with the target nuclei alters the direction of the electrons and causes nuclear energy loss. The electronic and nuclear energy losses suffered by the L-threonine molecule by the incident of $6 \mathrm{MeV}$ electrons were calculated as $(d E / d X)_{E}=1.84 \times$ $10^{6} \mathrm{eV} \cdot \mathrm{cm}^{2} / \mathrm{g}$ and $(d E / d X)_{N}=8.98 \times 10^{4} \mathrm{eV} \cdot \mathrm{cm}^{2} / \mathrm{g}$ using ESTAR program [102]. The range of the $6 \mathrm{MeV}$ electrons was also calculated as $2.2 \mathrm{~cm}$, which is too greater than the thickness of the samples (few millimeters) used for the irradiation measurements.

The mean excitation energy of the molecular crystal was also estimated as $72.5 \mathrm{eV}$. Hence, electrons with energies greater than a few $\mathrm{MeV}$ induce substantial permanent damage via displacement of the lattice atoms that results in a large number of lattice vacancies. The variation of electronic and nuclear stopping powers with electron energies suffered by the molecular crystal has been shown in Figure 39.

3.6.1. Material Characterization. The irradiated crystals were subjected to different measurements like electron paramagnetic resonance and micro-Raman analysis using Varian EPR - E112 $\times$ band $(9 \mathrm{GHz})$ spectrometer and Renishaw Invia Raman spectrophotometer by employing a $10 \mathrm{~mW}$ Argon ion laser at room temperature. Thermomechanical measurements of the irradiated crystals were carried out using Mettler TA 3000 TMA analyzer. Surface defects created by irradiation were studied by tapping mode atomic microscopy using a multimode SPM with nanoScope IIIa controller acquired from Digital/Veeco instruments Inc. Thermal stability of the irradiated crystal has also been studied by differential scanning calorimetry using NETZSCH Geratebau GmbH Thermal analyzer.

UV-Vis-NIR spectra of unirradiated and irradiated Lthreonine were recorded using Varian Cary 5E spectrophotometer in the wavelength range $200-1200 \mathrm{~nm}$. Dielectric and simultaneous TGA-DTA thermal measurements were performed using a Solartron impedance analyzer and NETZSCH-Geratebau GmbH thermal analyzer. Kurtz-Perry powder SHG test was also studied for the polycrystalline samples of uniform sizes of unirradiated and irradiated Lthreonine.

3.6.2. Electron Paramagnetic Resonance. The crystal structure of the compound is stabilized by a three-dimensional network of $\mathrm{N}-\mathrm{H} \cdots \mathrm{O}$ and $\mathrm{O}-\mathrm{H} \cdots \mathrm{O}$ bands through carboxylate oxygens. The stable free radical produced by electron irradiation is due to the breaking $\mathrm{C}-\mathrm{NH}_{3}$ bond leading to an unpaired electron spatially extending through $\mathrm{CH}_{3}$ and $\mathrm{CH}$ groups. The deaminated free radicals $\left(\mathrm{CH}_{3} \mathrm{CH}\right.$ $(\mathrm{OH}) \mathrm{CH} \mathrm{COOH})$ produced in the irradiated samples have a good EPR signal with a good response to the absorbed dose delivered to the samples. Formation of the deaminated radical due to the effect of electron irradiation is shown in Figure 40. EPR spectrum of polycrystalline L-threonine at room temperature (298 K) is shown in Figure 41.

Diphenyl picryl hydrazyl (DPPH) was used as standard field marker. The spectrum composed of six lines and it is due to the coupling of an unpaired electron located at the C-2 carbon radical site with 3 protons of $\mathrm{CH}_{3}$ group and 


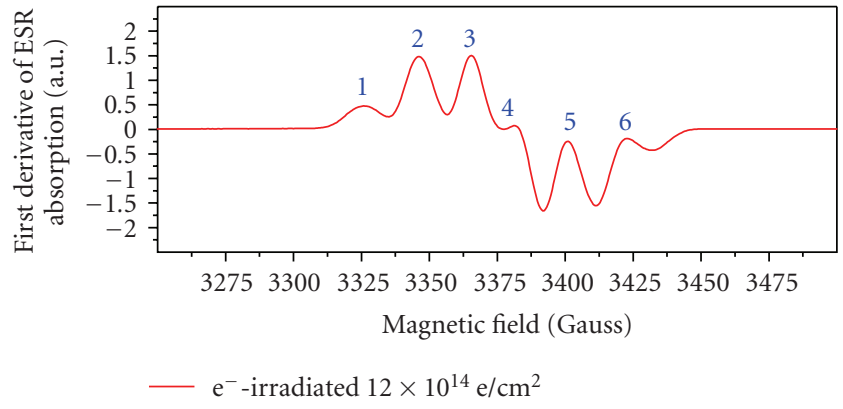

(a)

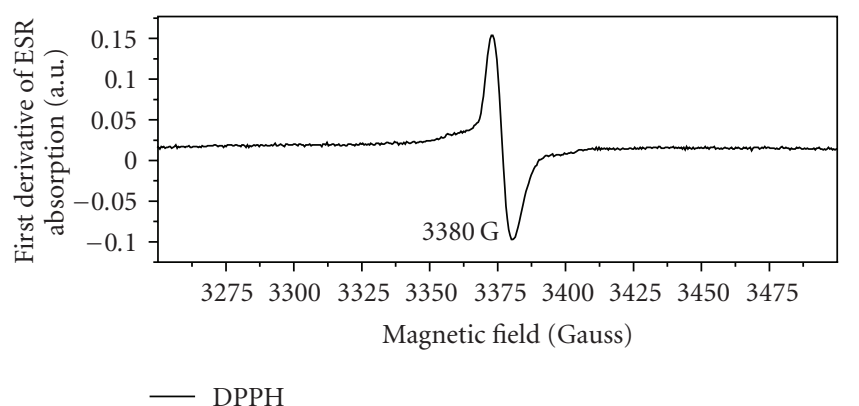

(b)

FIGURE 41: ESR spectrum of electron-irradiated $\left(12 \times 10^{14} \mathrm{e} / \mathrm{cm}^{2}\right)$ L-threonine crystal.

two protons of $\mathrm{CH}$ group through hyperfine interaction. As the $\mathrm{OH}$ proton attached to $\mathrm{C}-3$ carbon atom is not an immediate neighbourhood of the radical site, an EPR signal due to this proton is absent in the spectrum. Winkler et al. [103] reported that the integrated intensity of the room temperature EPR signals in irradiated L-alanine is used as a precise and stable dosimeter. Since the radical concentration of L-threonine is more than the L-alanine crystal, it can be considered as a better candidate for the radiation dosimetric applications. From EPR spectra, the $\mathrm{g}$ factor of the free radical formed by the electron irradiation was found to be 2.0027 .

3.6.3. Micro-Raman Analysis. Micro-Raman spectra were recorded for unirradiated and irradiated single crystals of L-Threonine at various fluences. The presence of four molecules per unit cell gives four additional lattice modes. The entire spectra can be divided into two types of modes, (i) intramolecular vibrations (stretching modes) and (ii) intermolecular vibrations (lattice modes). The modes around $2900 \mathrm{~cm}^{-1}$ and $2200 \mathrm{~cm}^{-1}$ shown in Figure 42 are intermolecular stretching vibrations of $\mathrm{CH}_{3}$ and $\mathrm{CH}$ groups. The modes in the range $100-400 \mathrm{~cm}^{-1}$ are attributed to the lattice vibrations. A peak at $3180 \mathrm{~cm}^{-1}$ in the unirradiated spectrum corresponds to $\mathrm{NH}$ vibrations of $\mathrm{NH}_{3}{ }^{+}$. The absence of this peak in all irradiated specimen shows the isolation of ammonia molecule after irradiation. At all fluences, both lattice and stretching modes show enhanced intensities when compared with similar modes in unirradiated spectrum of L-threonine and this agrees well with the earlier reports on L-alanine single crystals [103].

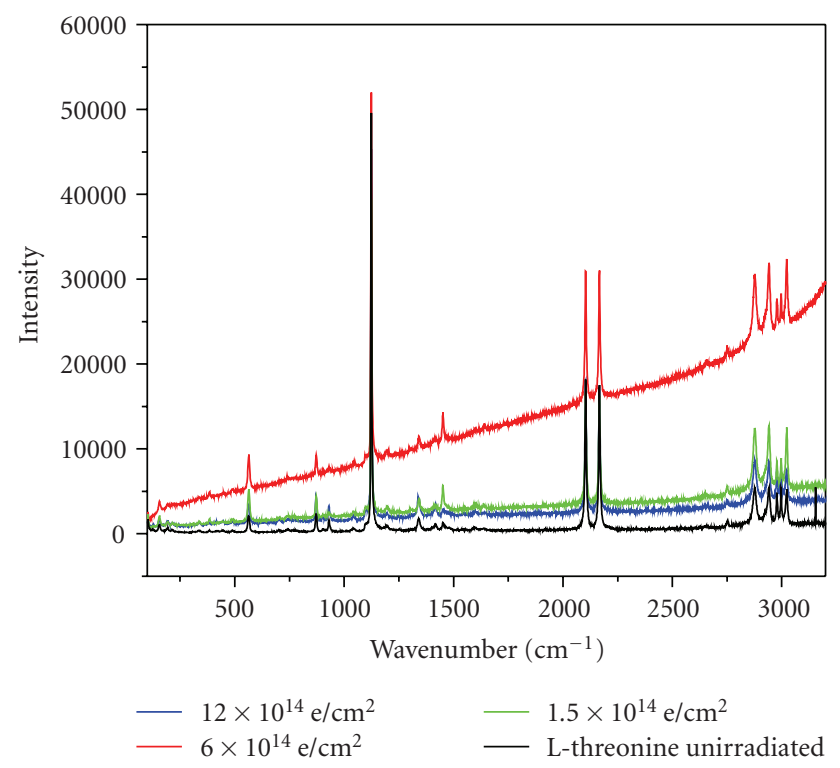

Figure 42: Micro-Raman spectra of pure and electron-irradiated L-threonine crystal at various fluencies.

3.6.4. Atomic Force Microscopy (AFM). AFM topographical images of L-threonine single crystals irradiated by $6 \mathrm{MeV}$ electrons with higher and lower fluences are presented. The penetration of the swift electrons at different fluences in Lthreonine produces raised rims of height 1.62 and $3.86 \mathrm{~nm}$ for the fluences $1.5 \times 10^{14} \mathrm{e} / \mathrm{cm}^{2}$ and $1.2 \times 10^{15} \mathrm{e} / \mathrm{cm}^{2}$, respectively. These raised rims that appear behind on each particle track were oriented precisely in the direction of the azimuth along which the incident electrons penetrate the surface. This could be seen in the tapping mode AFM pattern shown in Figures 43 and 44. Occasionally bumbs were also observed on the surface of crystals. Roughness of the surface increases at lower fluence and decreases for the higher fluence studied. Roughness values were found to be 47.57 and $12.42 \mathrm{~nm}$ for fluences $1.5 \times 10^{14}$ and $1.2 \times$ $10^{15} \mathrm{e} / \mathrm{cm}^{2}$, whereas the unirradiated surface possesses a roughness of $7.34 \mathrm{~nm}$ [52]. The decrease in the roughness of the crystal at a higher fluence is due to sputtering of molecules at the impact sites. Kopniczky et al. [104] reported that when an ion or charged particle penetrates into the bulk, the craters formation would cease but a volume force can still give rise to the plastic deformation. Accordingly, we could not observe any craters formed after irradiation, instead raised tails and bulges are found on the surface of the crystal clearly indicating radiation-induced sputtering as well as amorphization on its surface. The dimensions of the hillocks formed after irradiation are also shown in the AFM image.

3.6.5. Thermomechanical Analysis. Thermomechanical analysis (TMA) was carried out for both unirradiated and irradiated $\left(1.2 \times 10^{15} \mathrm{e} / \mathrm{cm}^{2}\right)$ crystals of L-threonine in the temperature range $50-130^{\circ} \mathrm{C}$ at a heating rate of $5^{\circ} \mathrm{C} /$ minute in air atmosphere. The title compound crystallizes in an orthorhombic system and hence the principal expansion 

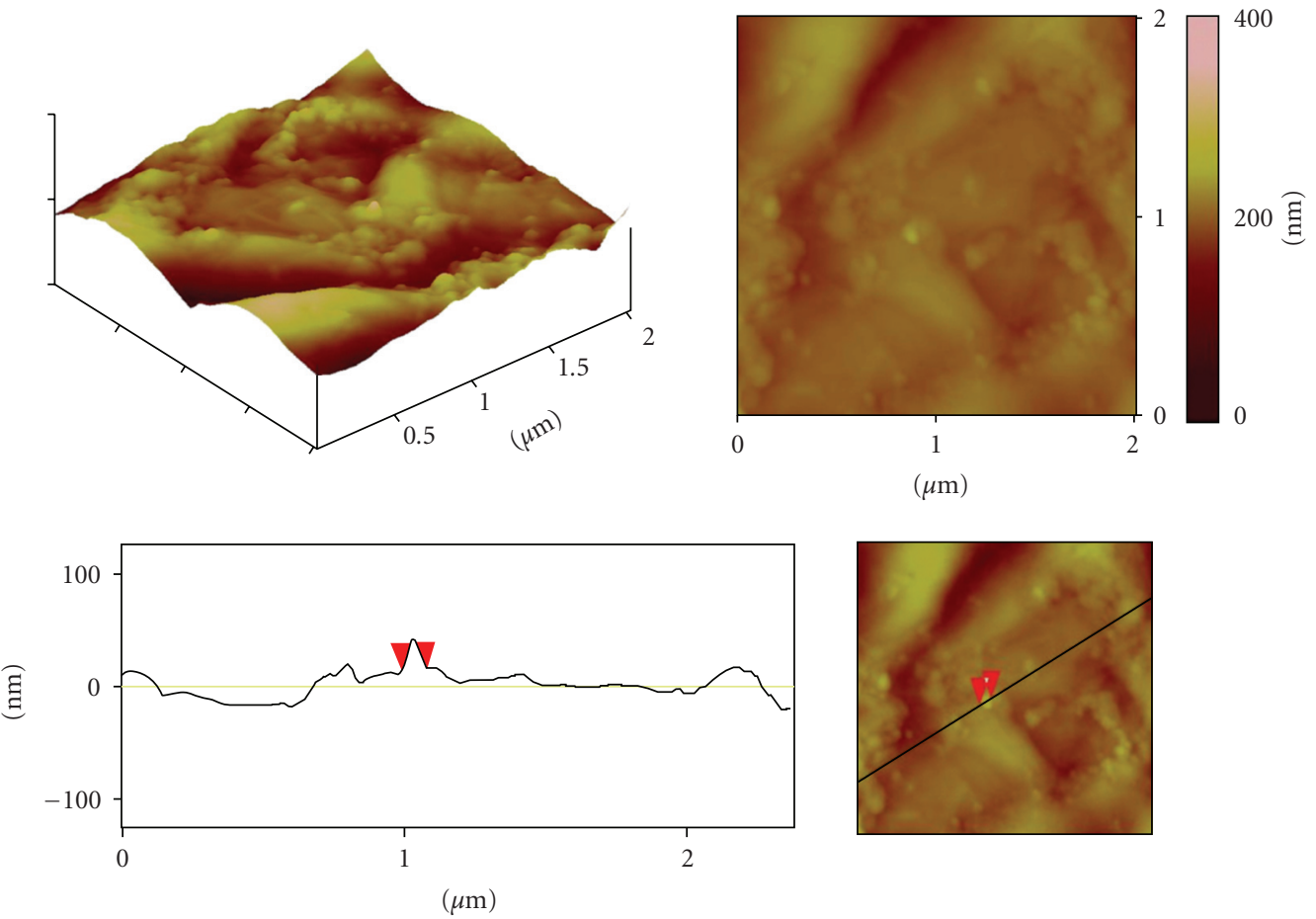

Figure 43: 3D and 2D AFM images of L-threonine crystal irradiated at $1.5 \times 10^{14} \mathrm{e} / \mathrm{cm}^{2}$.
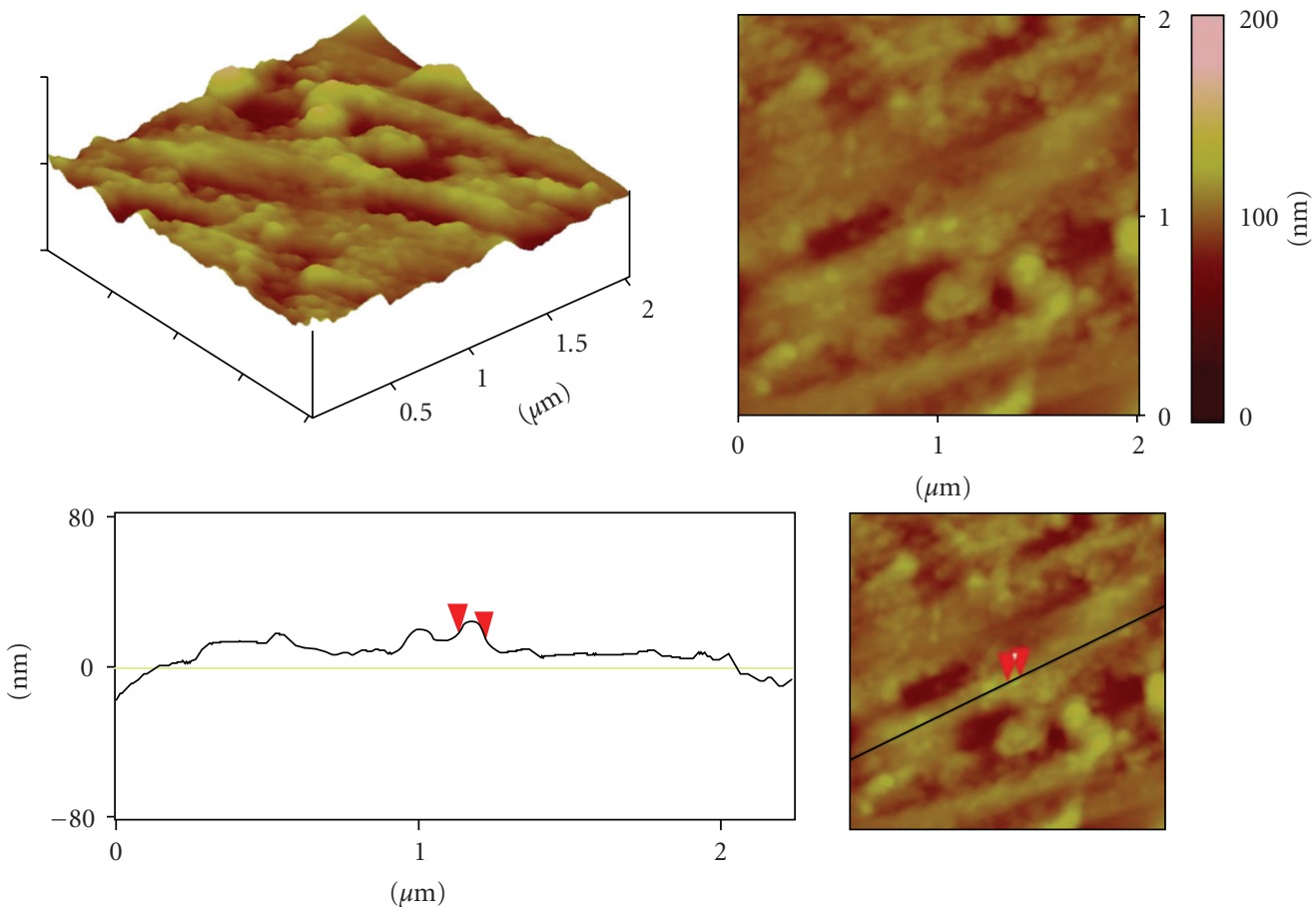

$(\mu \mathrm{m})$

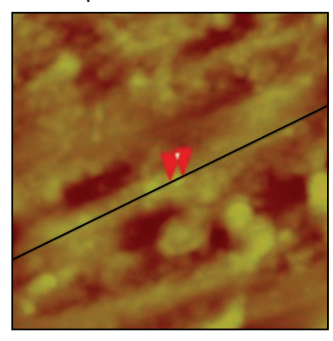

Figure 44: 3D and 2D AFM images of L-threonine crystal irradiated at $12 \times 10^{14} \mathrm{e} / \mathrm{cm}^{2}$.

coefficient tensor $\varepsilon_{1}, \varepsilon_{2}$, and $\varepsilon_{3}$ coincides with the crystallographic axes $a, b$, and $c$ [105]. Hence measurements have been made on all axes of the crystals before and after irradiation. Figures $45,46,47$, and 48 show the variation of linear strain, linear expansion, coefficient of linear expansion, and bulk expansion coefficient of L-threonine with temperature measured along the three mutually perpendicular crystal axes for both unirradiated and irradiated crystals. In all the axes, linear and bulk expansion coefficients of irradiated Lthreonine are found to increase for the temperature range 


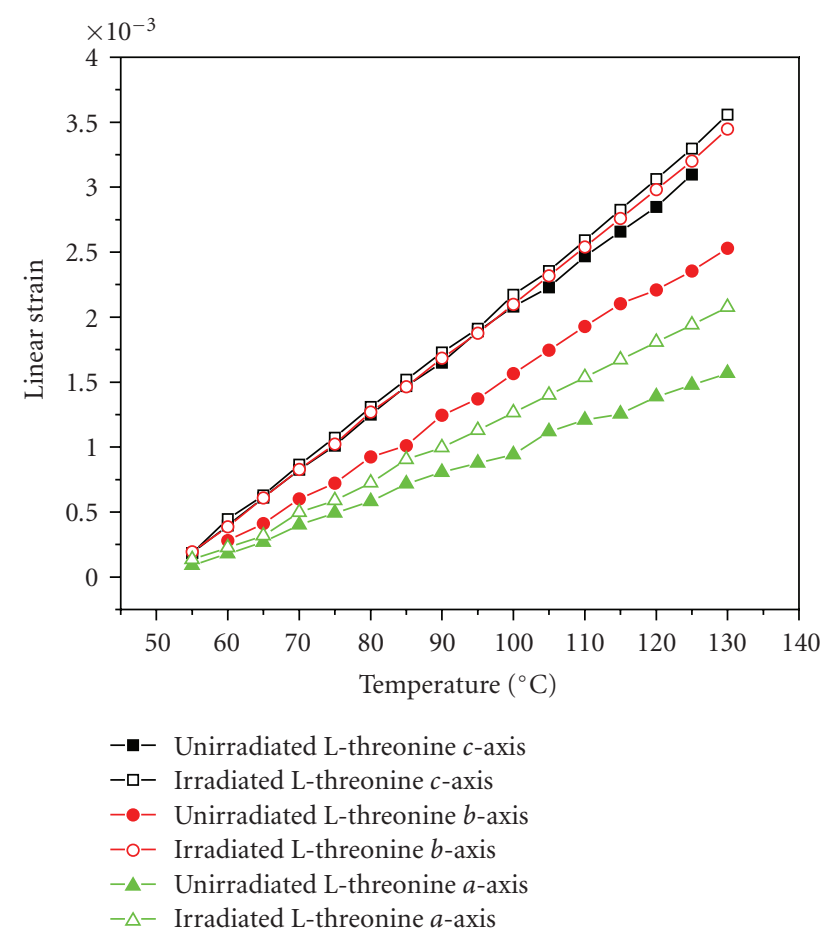

FIGURE 45: Linear strain versus temperature of pure and electronirradiated L-threonine crystal at $12 \times 10^{14} \mathrm{e} / \mathrm{cm}^{2}$.

TABLE 9: Linear and volume expansion coefficients of unirradiated and electron irradiated L-threonine single crystals at $12 \times$ $10^{14} \mathrm{e} / \mathrm{cm}^{2}$.

\begin{tabular}{lcc}
\hline Expansion coefficient in $\mathrm{K}^{-1}$ & Unirradiated & Irradiated \\
\hline$\alpha_{3}$ & $40.64 \times 10^{-6}$ & $42.94 \times 10^{-6}$ \\
$\alpha_{2}$ & $30.85 \times 10^{-6}$ & $41.58 \times 10^{-6}$ \\
$\alpha_{1}$ & $19.44 \times 10^{-6}$ & $24.93 \times 10^{-6}$ \\
$\alpha_{V}$ & $90.96 \times 10^{-6}$ & $109.45 \times 10^{-6}$ \\
\hline
\end{tabular}

studied. This can be explained through pressure pulse model [106, 107].

When high-energy particles incidents happen normally on the solid state matter, the electronic energy deposited by an incident electron relaxes depositing kinetic energy into the nuclear system. This may occur through coulomb explosion along the particle track. Alternatively, secondary electrons emitted from the tracks may excite the surrounding molecules and thus cause localized expansion of the molecular crystal.

Mean values of linear $(\alpha)$ and bulk expansion coefficient $\left(\alpha_{v}\right)$ of L-threonine before and after irradiation are shown in Table 9.

3.6.6. Differential Scanning Calorimetry (DSC). The thermal strength of the irradiated crystal has been investigated through DSC measurements in the temperature range $30-400^{\circ} \mathrm{C}$ at a heating rate of $5^{\circ} \mathrm{C} /$ minute in nitrogen atmosphere. From Figure 49 (a), it is clear that a single endothermic peak observed at $254.2^{\circ} \mathrm{C}$ corresponds to

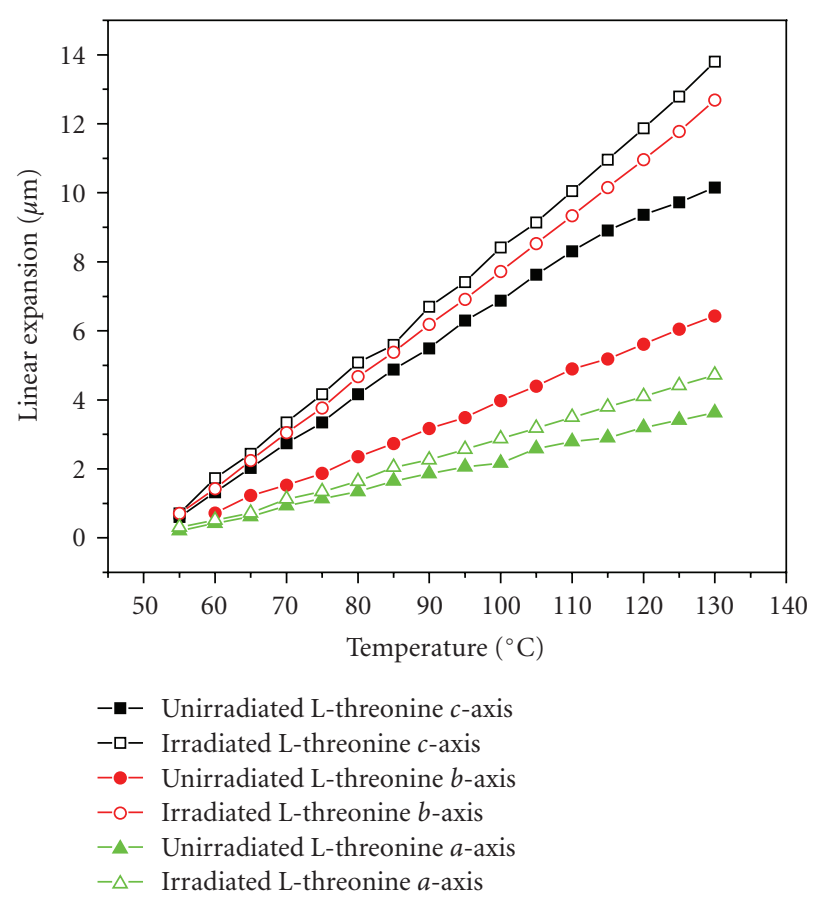

FiguRE 46: Linear expansion versus temperature of pure and electron-irradiated L-threonine crystal at $12 \times 10^{14} \mathrm{e} / \mathrm{cm}^{2}$.

the decomposition temperature of the crystal. But for an unirradiated crystal, this endothermic thermal event extends to a higher temperature of $265.2^{\circ} \mathrm{C}$. The decrease in the thermal stability of the irradiated crystal may be attributed to the breaking of $\mathrm{C}-\mathrm{N}$ bond and subsequent formation of the free radicals.

Simultaneous thermogravimetric and differential thermal analysis (TGA-DTA) was carried out for irradiated single crystals of L-threonine in the temperature range $30-700^{\circ} \mathrm{C}$ at a heating rate $5^{\circ} \mathrm{C} /$ minute in nitrogen atmosphere. A sharp weight loss and an endothermic thermal event at $253.4^{\circ} \mathrm{C}$ in TGA and DTA shown in Figure 49(b) correspond to the decomposition temperature of the irradiated L-threonine single crystals.

3.6.7. UV-Vis-NIR Spectral Measurements. UV-Vis-NIR spectra of unirradiated and irradiated L-threonine at various electron fluences of $3 \times 10^{14}, 6 \times 10^{14}$, and $9 \times 10^{14} \mathrm{e} / \mathrm{cm}^{2}$ are shown in Figure 50. The thicknesses of all the samples used were of the same dimensions. It was found that electron irradiation of L-threonine leads to a shift in the optical absorption edge indicating a lowering of energy gap. Absorption edges could not be seen for the unirradiated L-threonine and in irradiated crystals at lower fluence of $3 \times 10^{14} \mathrm{e} / \mathrm{cm}^{2}$ as they lie in the range $190-200 \mathrm{~nm}$. For higher electron fluences, $\lambda_{\max }$ shifts well and the decrease in energy gap might be due to the increase in the conductivity because of the following reasons. Formation of $\bullet \mathrm{CH}$ and deaminated radicals $\left(\bullet \mathrm{NH}_{2}\right)$ produced after irradiation were trapped in the crystal lattice and also the concentration of free electron radicals is proportional to radiation dose absorbed by the sample [103] as in the case of L-alanine 


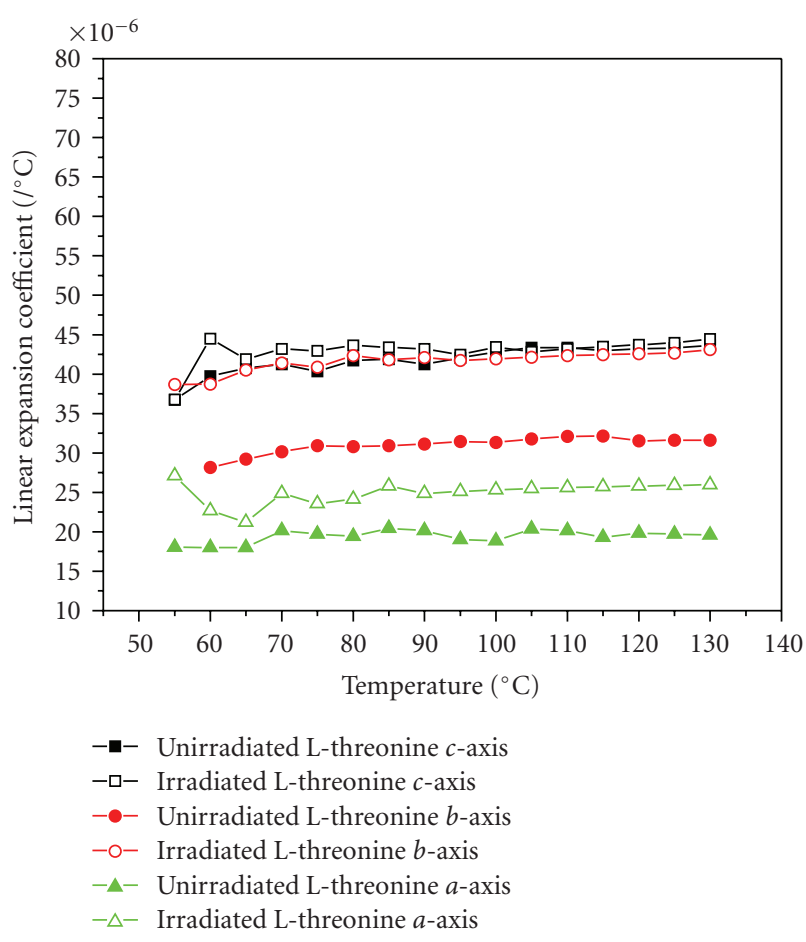

FIgURE 47: Coefficient of linear expansion versus temperature of pure and electron-irradiated L-threonine crystal at $12 \times 10^{14} \mathrm{e} / \mathrm{cm}^{2}$.

single crystals [108]. The increase in the conductivity lowers the energy gap and hence shifts the absorption edge towards the higher wavelength side. The results are in line with the reported works on similar studies [108-110].

3.6.8. Dielectric Studies. Dielectric permittivity $\left(\varepsilon_{r}\right)$ and dielectric loss $(\tan \delta)$ measurements were carried out in the frequency range $1 \mathrm{kHz}-1 \mathrm{MHz}$ at room temperature for unirradiated and irradiated single crystals of $\mathrm{L}$-threonine for the fluences of $1.5 \times 10^{14}, 3 \times 10^{14}$, and $6 \times 10^{14} \mathrm{e} / \mathrm{cm}^{2}$. It was found that dielectric constant of the irradiated crystal decreases with increase in the electron fluence. The dielectric constant was measured as 64 for the sample irradiated at of $6 \times 10^{14}$ [111] whereas the unirradiated crystal shows a relative permittivity as 103 for the same frequency of $0.1 \mathrm{MHz}$. As expected, the dielectric loss decreases at all electron fluences and it was found to have negligible values for the frequency range studied. As reported in UV studies, on irradiation there is an increase in the concentration of the free radicals and that leads to an increase in electrical conductivity of the crystal and hence a decrease in dielectric constant on increasing the electron fluence is observed. The increase in the conductivity of polypropylene on $2 \mathrm{MeV}$ electron irradiation [109] also supports this reported data. The results have been shown in Figures 51 and 52.

3.6.9. Kurtz-Perry Powder SHG Studies. Powder SHG efficiencies of both unirradiated and irradiated polycrystalline samples of L-threonine were measured using a laser source of wavelength $1064 \mathrm{~nm}$ with a pulse width of $10 \mathrm{~ns}$ at a repetition rate of $10 \mathrm{~Hz}$. The average particle sizes of all

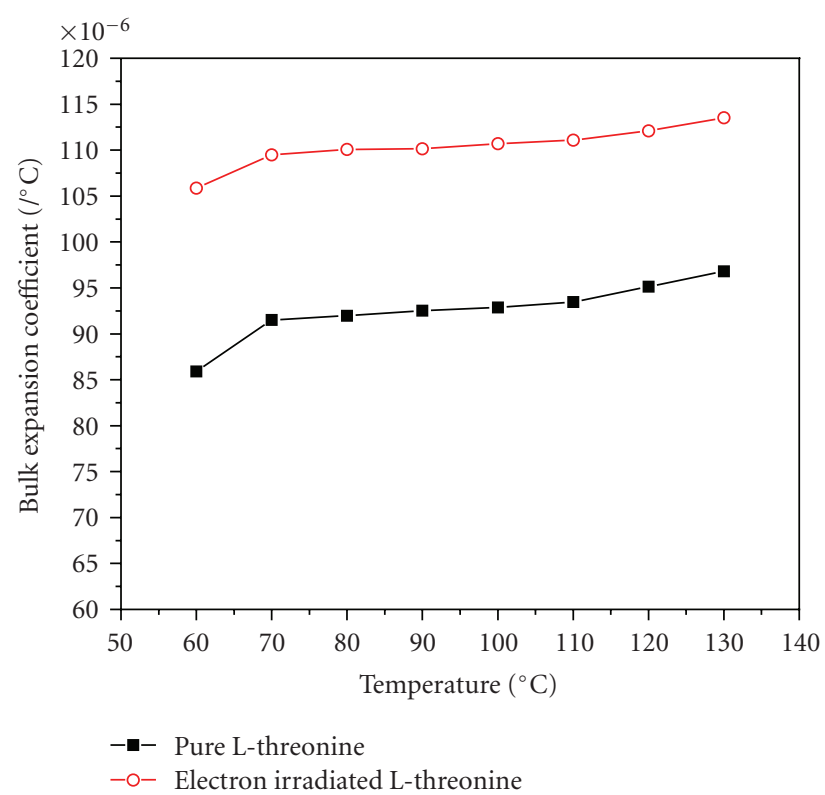

FIGURE 48: Bulk expansion coefficient versus temperature of pure and electron-irradiated L-threonine crystal at $12 \times 10^{14} \mathrm{e} / \mathrm{cm}^{2}$.

samples were found to be similar. It was found that no appreciable change in the SHG conversion efficiency was observed at all fluences studied. However, a slight increase in the SHG output was noticed at the higher fluence studied.

3.7. Lithium Ion Irradiation Effects on L-Threonine Single Crystals. Ion beam analysis techniques are very attractive for a range of structural and compositional characterization of solids [112]. However, these techniques typically use $\mathrm{MeV}$ light ions, which often produce understandable lattice disorder in insulators. Thus, for a successful application of ion beam analysis techniques for studies of impurities and defects in solids, it is crucial to ascertain the lattice disorder produced in materials by ion bombardment. Ion irradiation studies in solids are also important for better understanding of fundamental defect-related properties. Ion beam defect processes in crystals are currently limited to the investigations on KDP, barium strontium borate and methyl hydroxybenzoate [113-117]. Sreeramana Aithal et al. [118] have reported that $100 \mathrm{MeV} \mathrm{Ag}^{14+}$ ion irradiation on para-hydroy acetobenzene enhances its dielectric constant by 13 times and its SHG efficiency was also marginally affected. KeV ion beam irradiation effects of L-alanine [119] and L-threonine [120] using $\mathrm{N}^{+}$and $\mathrm{He}^{+}$ions have already been studied. In both cases, ion decomposition produces molecular emissions of $\mathrm{CO}_{2}, \mathrm{H}_{2}$ and $\mathrm{NH}_{3}$. Keeping in view of various reports, swift ion irradiation effects of $\mathrm{L}$-threonine single crystals and the influence on its physical properties have been concentrated.

3.7.1. Ion Irradiation. Crystals with well-defined morphology were cut into pieces of dimensions of $5 \times 5 \times 1 \mathrm{~mm}^{3}$ and polished using alumina paste. $\mathrm{Li}^{3+}$ ions of $50 \mathrm{MeV}$ from the 15 UD pelletron [53] at Inter University Accelerator 


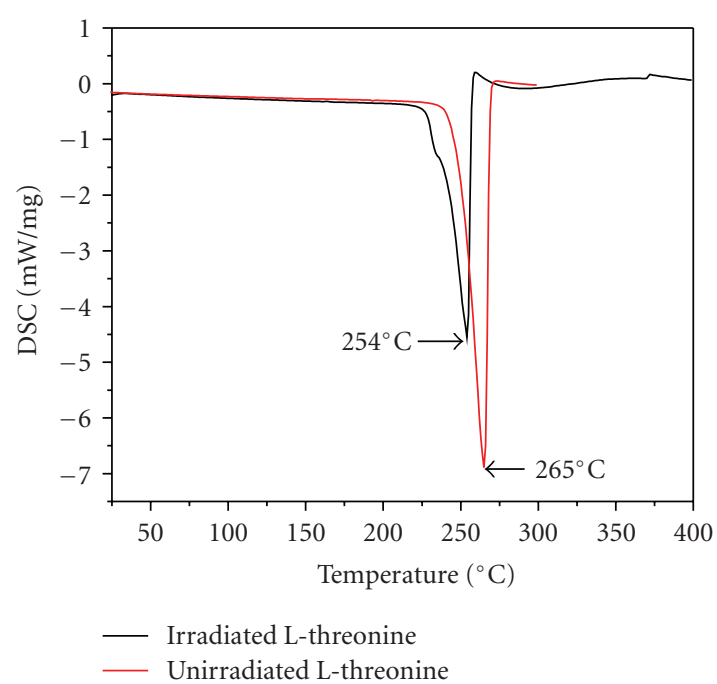

(a)

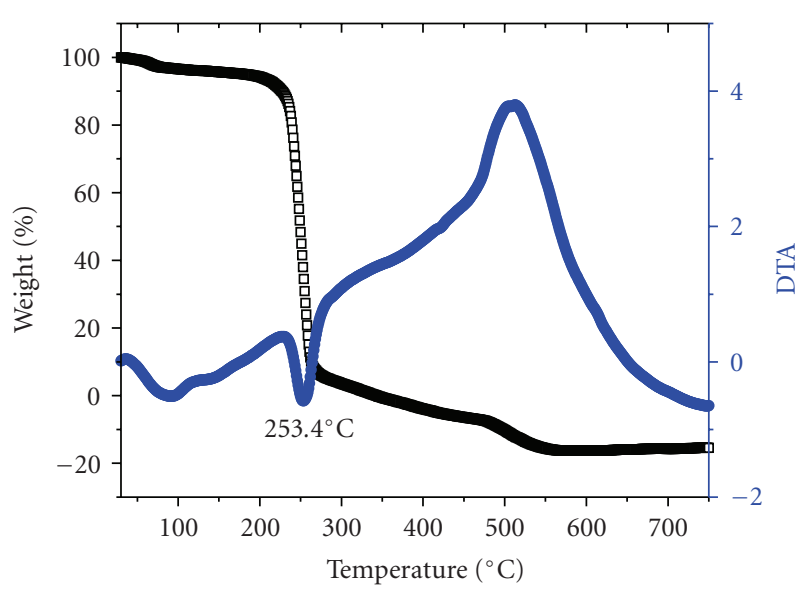

(b)

FIGURE 49: (a) DSC curves of unirradiated and electron-irradiated L-threonine crystal at $12 \times 10^{14} \mathrm{e} / \mathrm{cm}^{2}$. (b) simultaneous TGA-DTA curve for electron-irradiated L-threonine.

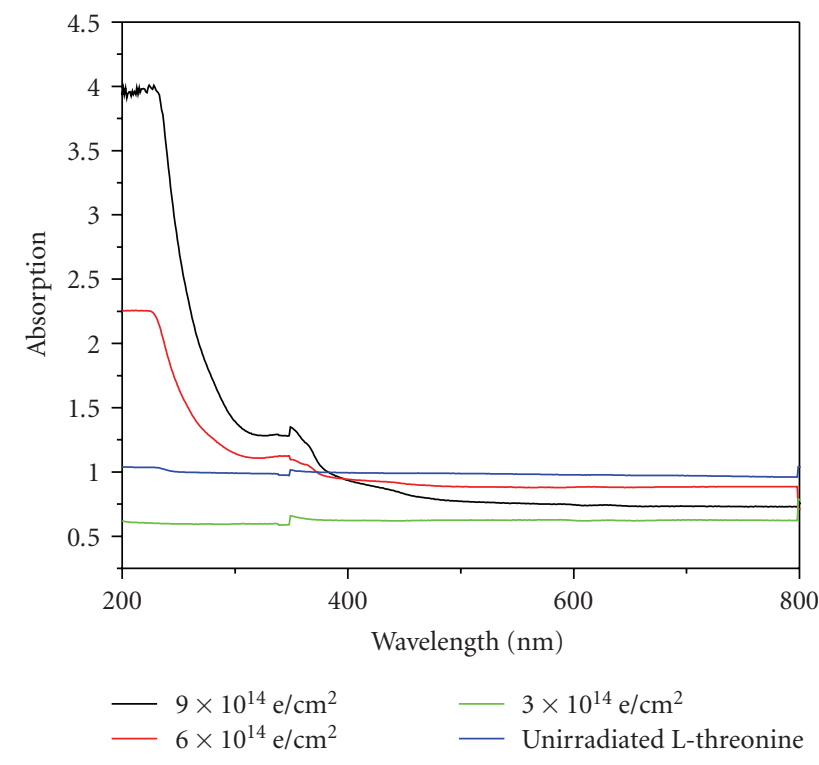

FIGURE 50: UV-Vis-NIR spectra of pure and irradiated L-threonine.

Centre (IUAC) New Delhi, India were allowed to incident on the sample mounted inside a $1.5 \mathrm{~m}$ diameter scattering chamber. The incident beam current was about 2 particle nano amperes (pna). The vacuum in the chamber during the experiment was kept at $8 \times 10^{-7}$ mbar. The crystals have been irradiated at various ion fluences namely $1 \times 10^{10}, 1 \times 10^{11}$ and $1 \times 10^{12} \mathrm{Li}^{3+}$ ions $/ \mathrm{cm}^{2}$ so as to study the fluence dependence on various physiochemical properties.

Swift heavy ions (SHI) produce intense radiation damage in all materials in which they traverse. The interaction between SHI and bulk materials lead to specific effects due to huge amounts of electronic energy $(d E / d x)_{E}$ being deposited on the target electrons by incident ions. In contrast projectile/target nuclei collision results in so called nuclear

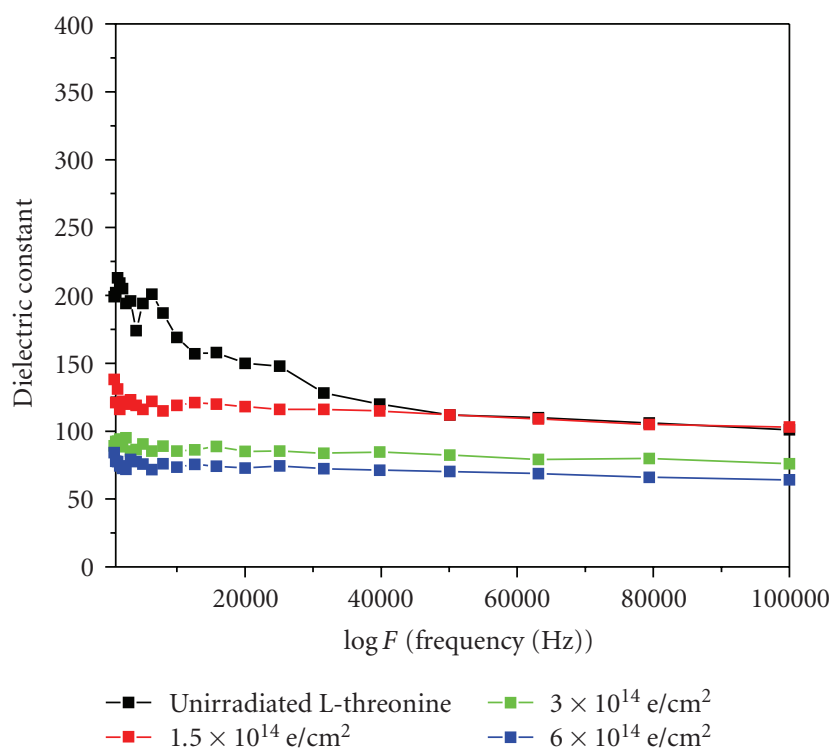

FIGURE 51: Dielectric constant of L-threonine for various electron fluencies.

energy transfer $(d E / d x)_{N}$ which plays a lesser role for energetic heavy ions. These effects have been studied for a long time in the case of insulators $[121,122]$. Both thermal spike [123] and pressure pulse/shock wave [106] models have been used for most materials to determine the sensitivity of materials to ion irradiations. Thermal spike model also predicts the threshold energy value of electronic energy loss of the ions to produce latent tracks in the materials. The latent tracks are supposed to be formed after rapid quenching of a cylindrical zone that has been melted along the ion path [124]. In heavily ionized track, due to Couloumb explosion secondary electrons emitted from the track excite the surrounding molecules showing a localized expansion in a solid and is projected in Figure 53. 


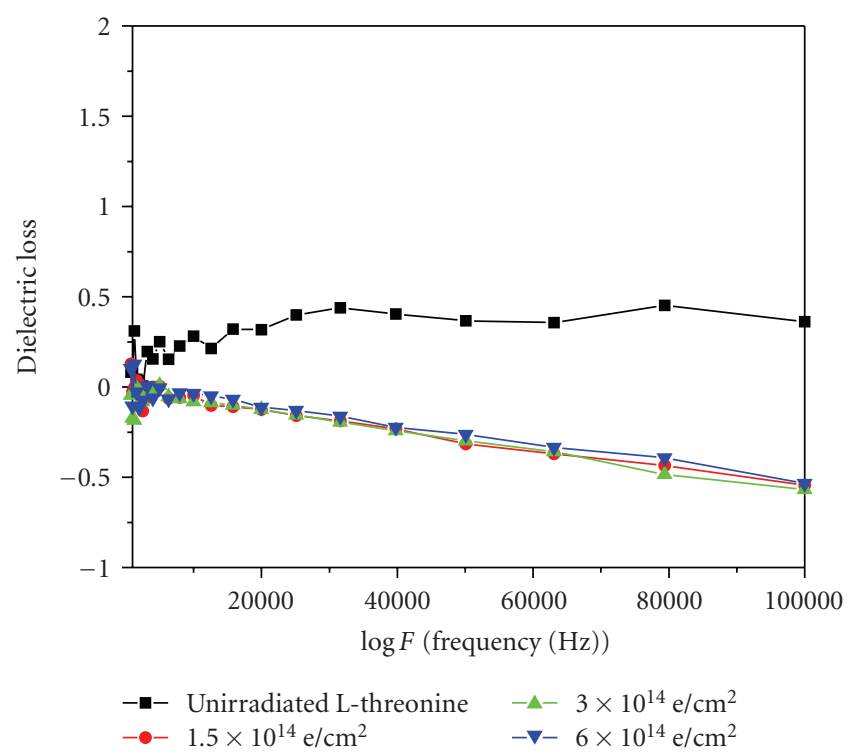

FIGURE 52: Dielectric loss of L-threonine for various electron fluencies.

The electronic and nuclear energy losses suffered by the $\mathrm{Li}^{3+}$ ion in $\mathrm{L}$-threonine crystals were calculated from transport of ion in matter (TRIM) program [125]. The projected range of $50 \mathrm{Mev} \mathrm{Li}^{+}$ions in L-Threonine crystal was determined as $411 \mu \mathrm{m}$. Electronic and nuclear stopping power of $\mathrm{Li}^{+}$ions in the title crystals are given by $(d E / d x)_{E}$ $=6.98 \mathrm{eV} / \AA$ and $(d E / d x)_{N}=3.63 \times 10^{-3} \mathrm{eV} / \AA$. Variation of electronic and nuclear energy losses of $\mathrm{Li}^{+}$ion for various incident energies is shown in Figure 54.

Characterization Studies. Ion irradiation produces modification in bulk, localized and surface properties of the materials depending upon the irradiation dose, energy and atomic number of the ion beam and also properties of the target materials. The irradiated biomolecules of L-threonine using $\mathrm{Li}^{3+}$ ion at various ion fluencies were subjected to various tools like X-ray diffraction (Bragg Brentano mode), UV-Vis-NIR Spectroscopy, Dielectric studies, Atomic Force Microscopy and Kurtz-Perry powder SHG measurements. Surface changes on irradiating the samples at various fluences were studied using a Bruker AXS D8 Advance X-ray diffractometer with $\mathrm{CuK} \alpha$ radiation. Transmittance spectrum of both unirradiated and irradiated crystals were recorded using Hitachi V-3300 UV-Vis-NIR spectrophotometer in the wavelength range $190-1100 \mathrm{~nm}$. Dielectric and AFM measurements on irradiated samples have been performed using solatron impedence analyser and multimode SPM nanoscope IIIa controller of Digital/Vecco instruments Inc. Powder SHG efficiencies for the irradiated samples of L-threonine were also measured for all ion fluences studied.

3.7.2. Ion-Induced Changes on the Topography of L-Threonine Single Crystals X-Ray Diffractometry. X-ray diffraction patterns of Pristine (unirradiated) and irradiated samples of Lthreonine were recorded in Bragg Brentano (BB) geometry where both the X-ray source and the detector move at equal angles. The scan speed was set at $0.02^{\circ} /$ minute and a crystalline sample was also rotated at $4 \mathrm{rpm}$ simultaneously. Figure 55 shows the X-ray diffractogram of L-threonine crystals irradiated at various $\mathrm{Li}^{3+}$ ion fluences.

As ion fluence increases the degree of crystallinity of the irradiated samples decreases. For an ion fluence of $1 \times 10^{12} \mathrm{ions} / \mathrm{cm}^{2}$, the surface of the crystal has lost its crystallinity completely and it shows radiation induced amorphization on the surface of the sample. The use BB mode in X-ray diffraction is more advantageous for single crystals as it varies the X-ray incidence angle and in the present case the angle of incidence varied upto $40^{\circ}$. From the density and absorption coefficient $(\alpha)$ of L-threonine the Xray penetration depths were calculated at different incident angles using X-ray depth evaluation program [126] and it is shown in Figure 56.

For an incidence angle of $40^{\circ}$, X-ray penetration depth was found to possess a maximum value of $843 \mu \mathrm{m}$. Though it roughly estimates the amorphization depth of L-threonine irradiated at the higher fluence, TRIM calculation showed that the maximum projected range of $\mathrm{Li}^{3+}$ ion for the given energy was only around $411 \mu \mathrm{m}$. From these observations, it has been perceived that amorphization depth exceeds the range $(R)$ of the ions and it was approximately twice that of $R$ value. In this range electronic stopping is the most significant process and a large amount of energy would have been transferred into a relatively small volume causing an electronic avalanche and subsequently extends the damage region in the crystals. Deposition of considerable amount of sputtered material on the surface of sample [127] also accounts for the increased thickness of the amorphized layer.

3.7.3. Atomic Force Microscopy. Figures 57(a)-57(c) show tapping mode normal incidence AFM images of $\mathrm{Li}^{3+}$ ion irradiated L-Threonine crystal at the fluences $1 \times 10^{10}, 1 \times 10^{11}$ and $1 \times 10^{12}$ ions $/ \mathrm{cm}^{2}$.

Samples bombarded at lower fluence $1 \times 10^{10}$ ions $/ \mathrm{cm}^{2}$ show raised rims of height $0.85 \mathrm{~nm}$ and width $129.29 \mathrm{~nm}$. For an ion fluence $1 \times 10^{11}$ ions $/ \mathrm{cm}^{2}$, craters were also formed with a depth of $0.5 \mathrm{~nm}$ and a width $169 \mathrm{~nm}$. Evaporation from the surface on a molecule-by-molecule basis could yield a crater. According to pressure pulse model, [106] if the impulse to a volume of material exceeds a critical value, then volume of material will get sputtered as a hemispherical sputtered volume has been predicated [128]. At the extremeties of the crater, the surface material does not receive enough momentum and highly energized underlying material attempts to eject. So a partial plastic deformation in the form of bulge is induced on either side of a crater. At a higher fluence of $1 \times 10^{12}$ ions $/ \mathrm{cm}^{2}$, materials would be receiving more than critical impulse and can easily escape leading to sputtering. The locations where the ion track is deeper, material receiving the critical impulse is stopped by the stationary material above resulting an observation of tail like permanent plastic deformation along the ion track which indicates that a hydrodynamic pressure pulse occurs in response to the electronically deposited energy. Similar observation has also been reported for L-valine crystals 


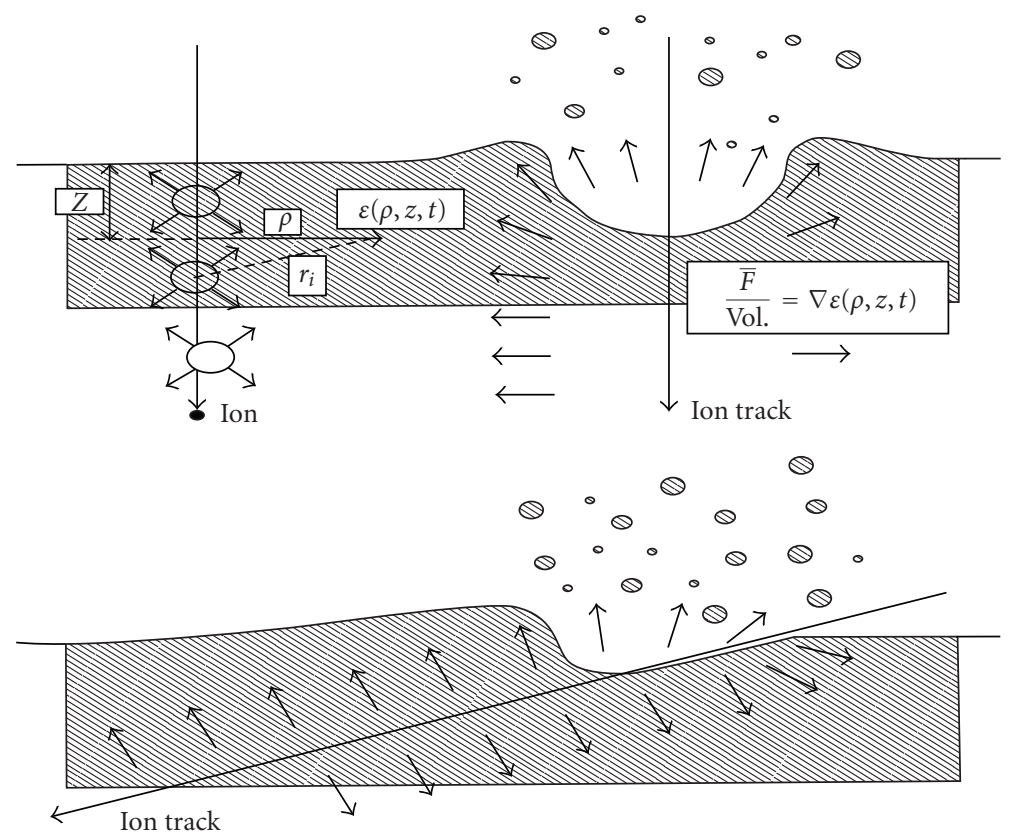

FIGURE 53: Schematic diagram of models of sputtering and plastic deformation due to a cylindrical pressure pulse.

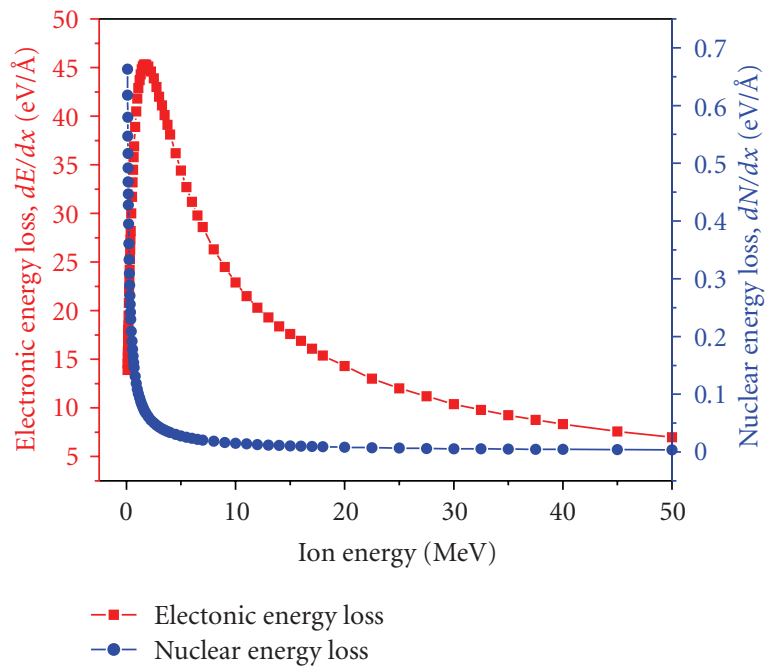

Figure 54: Nuclear and electronic energy losses for various input incident $\mathrm{Li}^{3+}$ ion energies in L-threonine single crystals.

$[104,129]$. The mean roughness values of the irradiated samples were $11.27,45.72$ and $18.66 \mathrm{~nm}$ for the ion fluences $10^{10}, 10^{11}$, and $10^{12}$ ions $/ \mathrm{cm}^{2}$ whereas the pristine sample has a roughness of $7.3 \mathrm{~nm} \mathrm{[130].} \mathrm{The} \mathrm{decrease} \mathrm{in} \mathrm{the} \mathrm{mean}$ roughness at the higher fluence is mainly due to sputtering of substantial amount of material per incident of $\mathrm{MeV}$ ion.

\subsubsection{Electrical, Thermal and Optical Properties of Lithium Irradiated L-Threonine Single Crystals}

Dielectric Studies. Dielectric permittivity and dielectric loss measurements were carried out for the irradiated crystals of L-threonine in the frequency range $1 \mathrm{kHz}$ to $1 \mathrm{MHz}$ at room temperature. Figures 58(a) and 58(b) show the dispersion of dielectric constant and dissipation factor with ion fluence. At lower fluence of $1 \times 10^{10} \mathrm{ions} / \mathrm{cm}^{2}$, the dielectric constant decreases to 62 at $1 \mathrm{MHz}$. The decrease in dielectric constant may be due to the formation of small fragments including single atoms. Some of these $\mathrm{H}, \mathrm{OH}$, $\mathrm{CH}$ and $\mathrm{CO}_{2}$, and so forth, are volatile and readily degass from the sample and simultaneous formation of very large number of free radicals allows the movement of unpaired electrons within the molecular dipoles and hence increases the electrical conductivity of the sample. At higher fluence of $1 \times 10^{12}$ ions $/ \mathrm{cm}^{2}$, the dielectric constant shoots up suddenly due to amorphization of the sample surface and it attains a steady state value of 191 for the entire frequency range studied. The dielectric loss values were found to be decreasing with the increase in ion fluences studied. It is well known that electro-optic coefficient $\left(r_{i j}\right)$ of any NLO molecular crystal depends on its dielectric constant and hence the irradiated crystals of L-threonine at a desired fluence can modulate its electro-optic properties. Thus ion irradiation on second order NLO materials can easily tune their $r_{i j}$ values.

3.7.5. UV-Vis-NIR Spectroscopy. UV-Vis-NIR spectra recorded for the unirradiated and irradiated crystals of L-threonine in the wavelength 190-1100 nm show that there is a slight shift in the absorption edge towards lower wavelength side for the higher fluence reflects the results obtained in the dielectric measurements. Percentage of absorption has also changed with the ion fluence and it is shown in Figure 59.

3.7.6. Thermal Analysis. Simultaneous thermogravimetric and differential thermal analysis (TGA-DTA) was carried out 


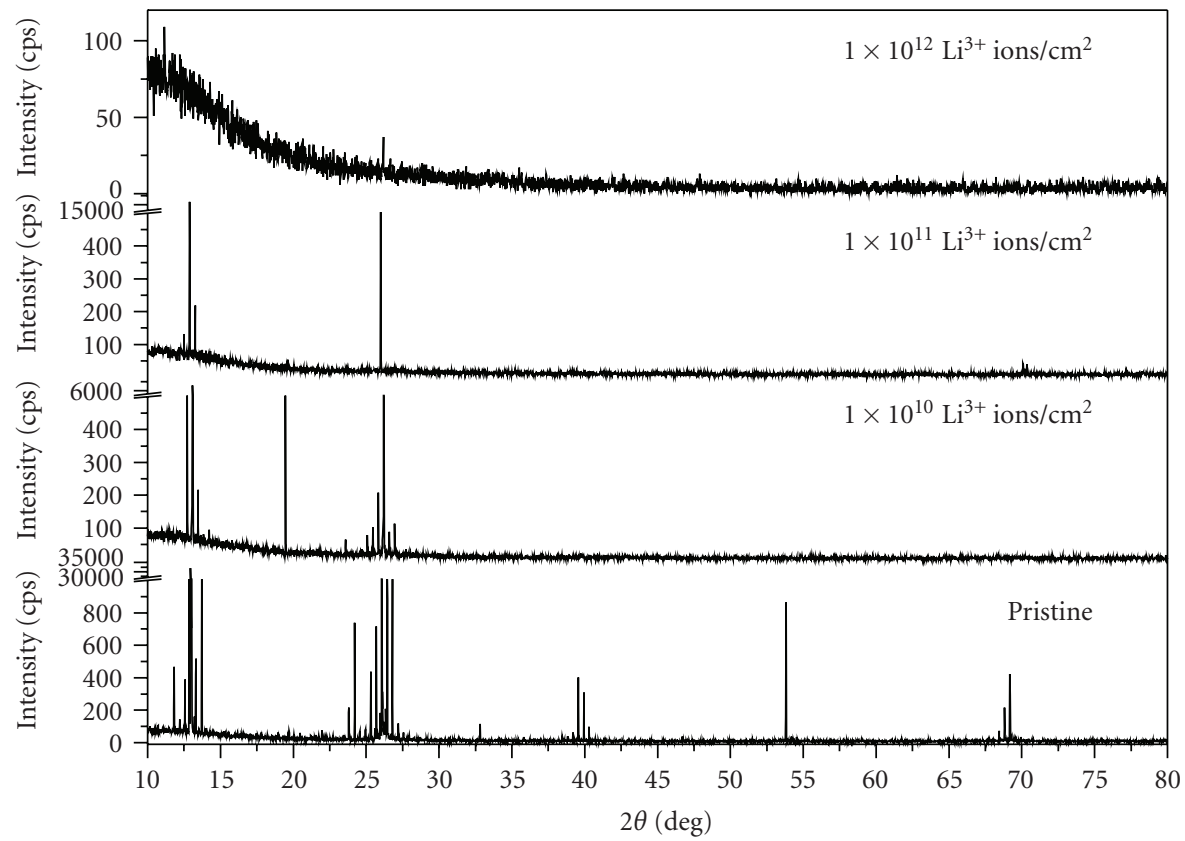

FIGURE 55: X-ray diffraction patterns of unirradiated and irradiated L-threonine single crystals at various ion fluencies.

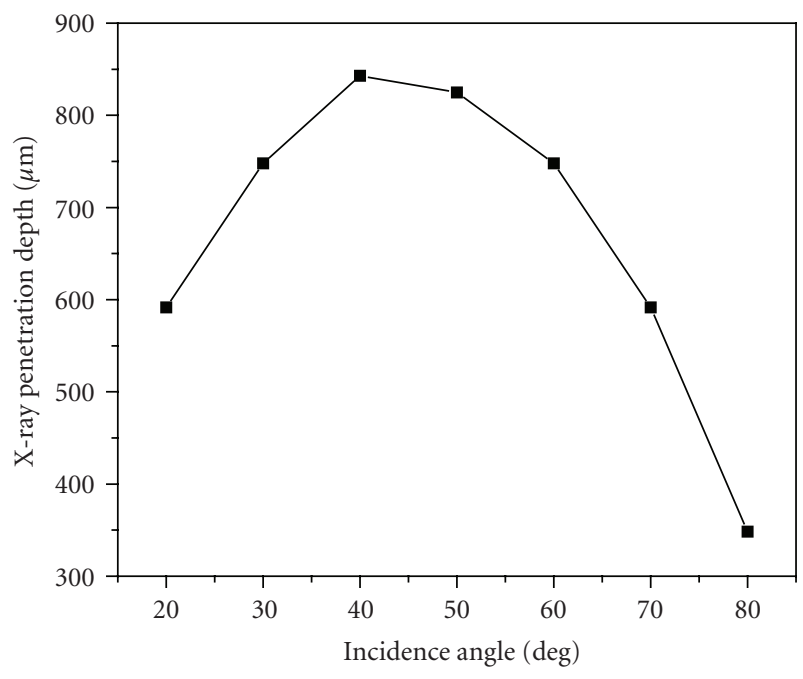

FIGURE 56: X-ray penetration depth in L-threonine crystals at various incident angles.

for the irradiated crystals of L-threonine $\left(1 \times 10^{11}\right.$ ions $\left./ \mathrm{cm}^{2}\right)$ in the temperature range $30-600^{\circ} \mathrm{C}$ at a heating rate of $5^{\circ} \mathrm{C} /$ minute in nitrogen atmosphere is shown in Figure 60 A sudden weight loss and an endothermic thermal event at $278.6^{\circ} \mathrm{C}$ in TGA and DTA shows the decomposition temperature of irradiated L-threonine, while for an unirradiated crystal, the decomposition was reported as $265.2^{\circ}$. The increase in thermal stability is due to decrease in the degree of crystallinity of the irradiated samples.

3.7.7. Kurtz-Perry Powder SHG Measurement. The second harmonic generation conversion efficiency of both unirradiated and irradiated polycrystalline samples of L-threonine were measured relative to KDP. The instrument used was $\mathrm{Nd}$ : YAG laser of wavelength $1064 \mathrm{~nm}$ with pulse width of $10 \mathrm{~ns}$ with a repetition rate of $10 \mathrm{~Hz}$. The average grain size of all the unirradiated and irradiated samples with different ion fluence was found to be same. At lower ion fluences, powder SHG efficiency of L-threonine shows no appreciable variation but for higher fluences $\left(10^{11}\right.$ and $10^{12}$ ions $\left./ \mathrm{cm}^{2}\right)$, SHG output drastically reduced to half of its original value. This may be due to the fact that higher dose of irradiation has considerably affected the second-order nonlinearity of the molecular crystal.

\section{Conclusions}

(1) The growth parameters governing the crystal growth process such as free energy formation, metastable zonewidth, induction period, and supersaturation of the solutions were found out experimentally. Bulk single crystals of L-threonine have been harvested under optimized growth conditions.

(2) The UV-Vis-NIR spectrum of the L-threonine crystal shows that the crystal is transparent even upto near IR region. The presence of various functional groups and the zwitterionic nature of the molecule were confirmed by FT-IR and FT-Raman spectra. TGA and DSC measurements show that the crystal decomposes at $262^{\circ} \mathrm{C}$. DSC also shows that no structural phase transition was observed in the Low-temperature region. Dielectric studies on the crystalline samples at various temperatures show that the temperature has influenced much on the dielectric property of the material. The dielectric constant was found to be 61 and 175 at $1 \mathrm{MHz}$ for 25 and $110^{\circ} \mathrm{C}$. Dielectric 


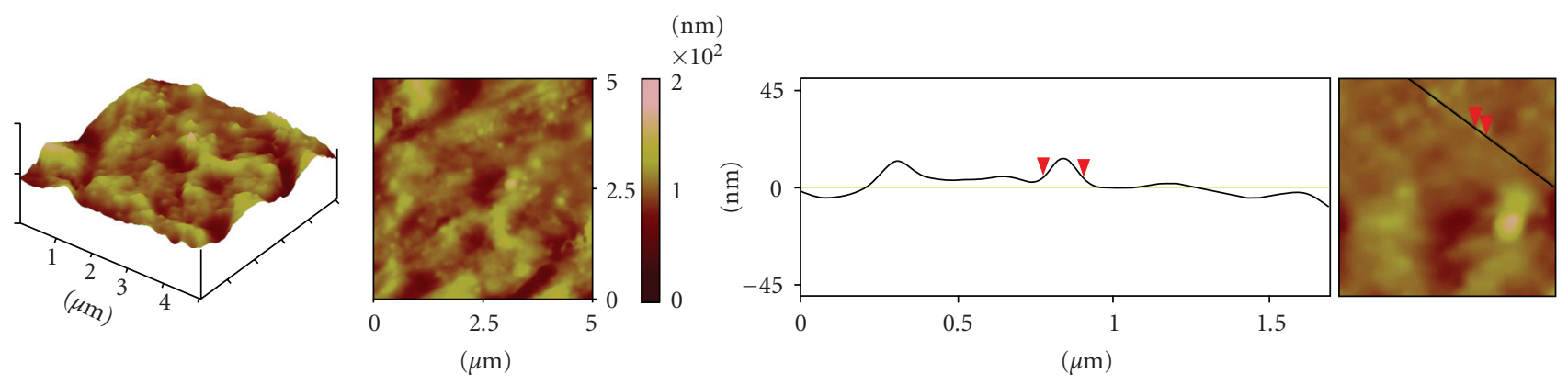

(a)
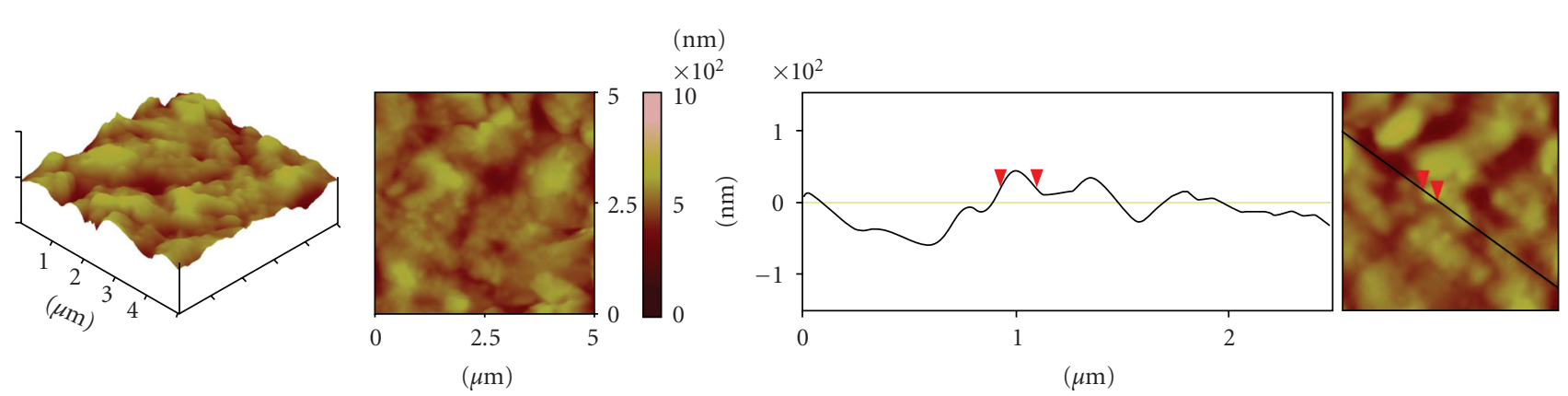

(b)
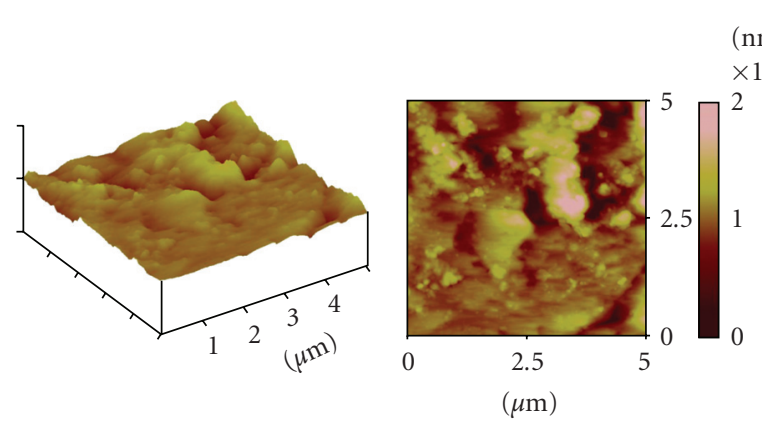

$(\mathrm{nm})$
$\times 10^{2}$

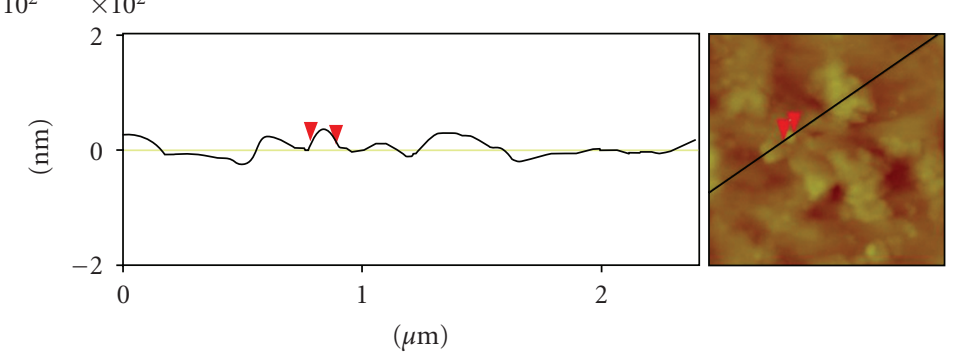

(c)

Figure 57: (a) 3D and 2D AFM images of irradiated L-threonine at $1 \times 10^{10}$ ions $/ \mathrm{cm}^{2}$. (b) 3D and 2D AFM images of irradiated L-threonine at $1 \times 10^{11}$ ions $/ \mathrm{cm}^{2}$, (c) $3 \mathrm{D}$ and $2 \mathrm{D}$ AFM images of irradiated L-threonine at $1 \times 10^{12} \mathrm{ions} / \mathrm{cm}^{2}$.

loss values were also found negligible at higher frequencies. Thermomechanical measurements on Lthreonine single crystals shows that the crystal possesses lower expansion coefficients when compared to many NLO single crystals. Microhardness studies reveals that the mechanical strength of the crystal may be feasible for device fabrication. The SHG efficiency was measured by Kurtz's powder technique and it is greater than that of KDP and many organic NLO crystals.

(3) Significant study on the L-threonine crystal grown at isoelectric $\mathrm{pH}$ shows the enhancement of powder SHG efficiency of crystal. HRXRD analysis reveals that the crystalline perfection of the crystal grown at isoelectric point was found to be much better than the crystals grown at other $\mathrm{pH}$ values.
(4) Deuteration of L-threonine single crystals shows that only $-\mathrm{NH}$ and $-\mathrm{OH}$ groups were replaced by deuterons. Methyl group protons remain unaffected. Powder XRD shows that on deuteration, crystalline quality has been improved considerably. Dielectric study reveals that deuteration increases the value of dielectric constant of L-threonine from 61 to 102 at $1 \mathrm{MHz}$. Deuteration has not changed the transmittance property in UV-Vis-NIR region of the grown crystal. Thermal analysis shows that thermal strength of deuterated crystal was slightly increased on deuteration. On deuteration, the powder SHG efficiency of L-threonine was also marginally affected.

(5) Three dimensional anharmonic oscillator model (AHO) has been used to evaluate SHG and electrooptic susceptibility of L-Threonine single crystals. 


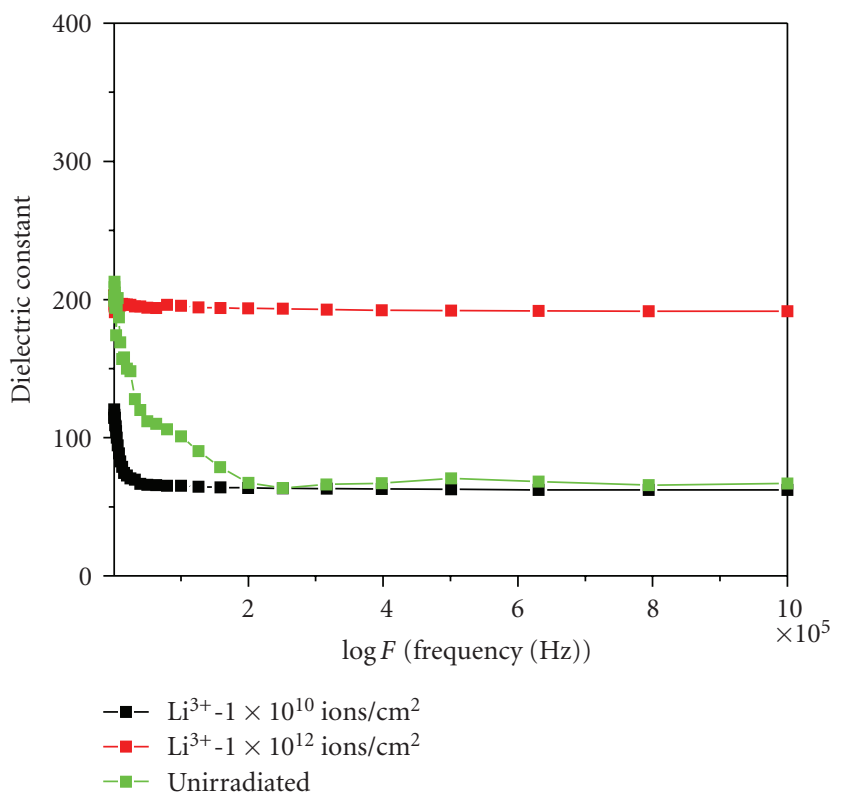

(a)

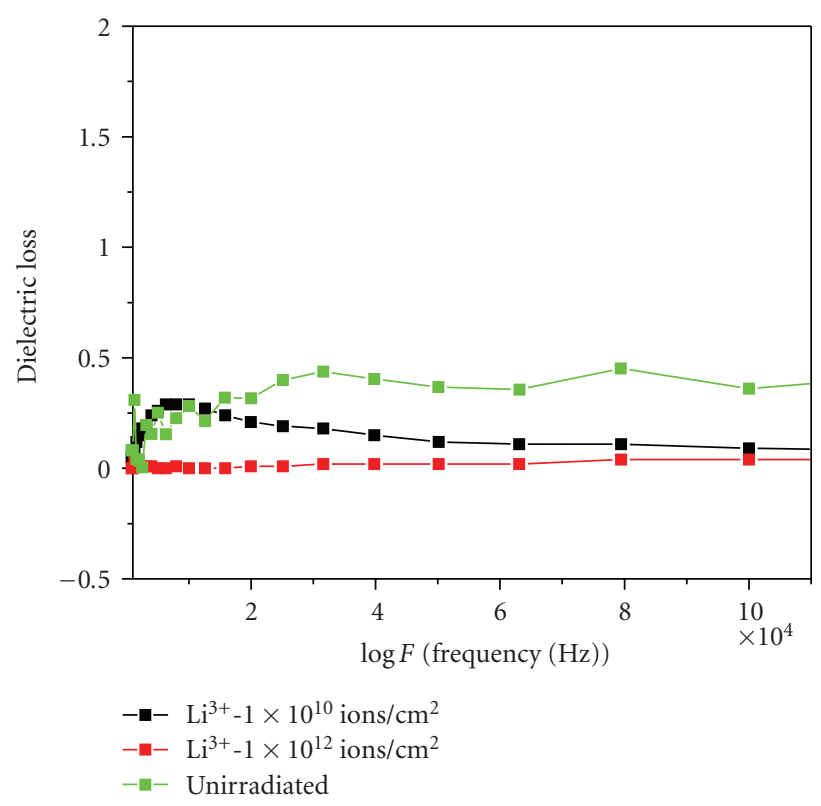

(b)

Figure 58: (a) Dielectric constant of unirradiated and irradiated L-threonine single crystals at various ion fluences. (b) dielectric loss of unirradiated and irradiated L-threonine single crystals at various ion fluencies.

Linear and the second order nonlinear SHG susceptibilities were found to decrease with the increase in wavelength. It was also found that Pockel's coefficient $\left(r_{63}\right)$ of L-threonine was comparable to Lalanine single crystals. It has also been demonstrated that the electronic contribution to the anharmonicity is itself sufficient to explain the NLO properties of Lthreonine.

(6) Single crystals of L-threonine irradiated by highenergy turn yellowish due to the presence of electronic defects in the gap (colour centres). The formation of an unpaired electron at C-2 carbon atom through $\mathrm{C}-\mathrm{N}$ bond breaking and the movement of the free electrons along the radical sites have been confirmed from electron paramagnetic resonance studies. EPR measurements also show that the stable free radicals produced after irradiation can be useful to act as an alternative candidate for L-alanine for radiation dosimetry applications.

From microraman studies, the absence of asymmetric $\mathrm{N}-\mathrm{H}$ stretching vibrations around $3180 \mathrm{~cm}^{-1}$ in all samples irradiated at different fluences confirms the isolation of ammonia molecule after irradiation. Both intramolecular and intermolecular stretching vibrations of L-threonine show enhanced intensities on electron irradiation and indicate as local probes for the presence of localized electrons in different parts of the molecules. Atomic force microscopy has provided a new and unique view of intersection of an electron track with a surface and has elucidated the response of a material to a dense microscopic track of deposited energy. Thermomechanical measurements show that both linear and bulk expansion coefficients of irradiated L-threonine possesses higher values when compared to

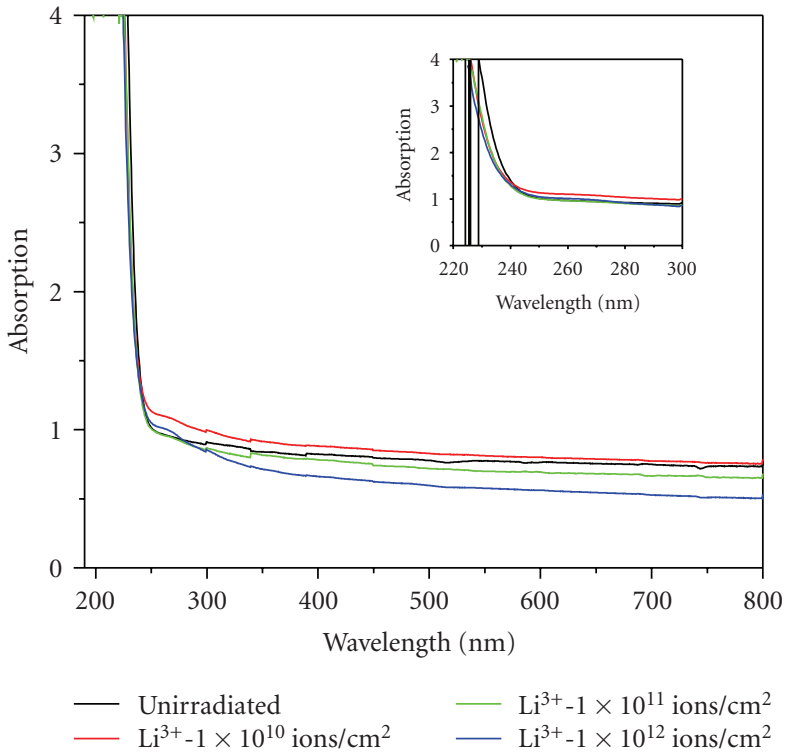

FIGURE 59: UV-Vis-NIR spectra of unirradiated and irradiated Lthreonine single crystals at various ion fluencies.

unirradiated crystals at all temperatures. Irradiation induced changes in thermal expansion coefficients may be attributed to the displacement damage produced by the higher nuclear energy loss. From DSC and TGA-DTA analyses, it has been found that electron irradiation reduces the thermal stability of the L-threonine single crystals.

UV-Vis-NIR spectra of electron irradiated crystals show that the optical band gap energy decreases with increase in the fluence. Dielectric permittivity of irradiated L-threonine was found to be decreased on increasing the electron fluence. This has been attributed to increase in conductivity of the 


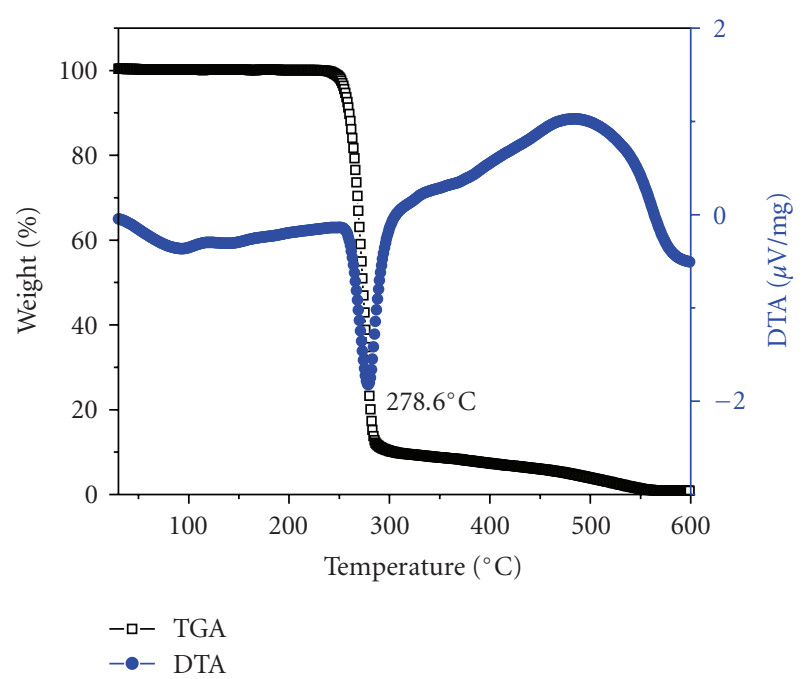

FIgURE 60: Simultaneous TGA-DTA of irradiated L-threonine single crystals at $1 \times 10^{11} \mathrm{ions} / \mathrm{cm}^{2}$.

solid due to the formation of free radicals. Dielectric loss values were also found to be decreased at all fluences studied. Kurtz-Perry powder SHG study shows that no change was observed at lower electron fluences but the second harmonic output increases for higher electron fluences.

(7) L-threonine single crystals were irradiated by $50 \mathrm{MeV}$ $\mathrm{Li}^{3+}$ ions at various ion fluences. X-ray diffraction study confirms the amorphization phenomena occurring on the surface of crystals at the higher fluence. Atomic force microscopy of irradiated crystals show that interaction of high-energy ion with soft bio-molecules leads to the ejection of intact molecules through sputtering mechanism. Tail like appearance in the AFM images at the higher fluences indicates the permanent plastic deformation on the surface of the crystals. Dielectric studies reveal that dielectric constant decreases at the lower fluence and increases suddenly for the higher fluences due to amorphization. Ion irradiation of L-threonine also shifts the absorption edge towards the lower wavelength side. Thermal strength of irradiated Lthreonine was found to be higher than that of unirradiated L-threonine. Powder SHG measurements show that the second harmonic output decreases for higher fluences and hence NLO properties of crystals were marginally affected by ion irradiation.

\section{Acknowledgments}

The authors wishes to thank Dr. R. Mohan, Department of Physics, Presidency College, Chennai-6, for his fruitful suggestions which prevails throughout this work. The author Dr. S. Gokul Raj wishes to thank DST-SERC for the award of Young Scientist under FTP scheme.

\section{References}

[1] D. S. Chemla and J. Zyss, Nonlinear Optical Properties of Organic Molecules and Crystals, Academic Press, New York, NY, USA, 1987.
[2] R. A. Hann and D. Bloor, Eds., Organic Materials for Nonlinear Optics, Royal Society of Chemistry, London, UK, 1989.

[3] M. H. Lycons, Ed., Materials for Nonlinear Optics and ElectroOptics, vol. 103 of Institute of Physics Conference Series, Institute of Physics, Bristol, UK, 1989.

[4] A. Yariv, Quantum Electronics, John Wiley \& Sons, New York, NY, USA, 1975.

[5] N. Bloembergen and Y. R. Shen, "Quantum-theoretical comparison of nonlinear susceptibilities in parametric media, lasers, and Raman lasers," Physical Review, vol. 133, no. 1, pp. A37-A49, 1964.

[6] S. B. Monaco, L. E. Davis, S. P. Velsko, F. T. Wang, D. Eimerl, and A. Zalkin, "Synthesis and characterization of chemical analogs of L-arginine phosphate," Journal of Crystal Growth, vol. 85, no. 1-2, pp. 252-255, 1987.

[7] T. Pal, T. Kar, G. Bocelli, and L. Rigi, "Synthesis, growth, and characterization of L-arginine acetate crystal: a potential NLO material," Crystal Growth and Design, vol. 3, no. 1, pp. 13-16, 2003.

[8] D. Xu, X. Q. Wang, W. T. Yu, S. X. Xu, and G. H. Zhang, "Crystal structure and characterization of a novel organic nonlinear optical crystal: L-arginine trifluoroacetate," Journal of Crystal Growth, vol. 253, no. 1-4, pp. 481-487, 2003.

[9] S. S. Terzyan, H. A. Karapetyan, R. B. Sukiasyan, and A. M. Petrosyan, "L-arginine nitrates," Journal of Molecular Structure, vol. 687, no. 1-3, pp. 111-117, 2004.

[10] M. K. Marchewka, S. Debrus, A. Pietraszko, A. J. Barnes, and H. Ratajczak, "Crystal structure, vibrational spectra and nonlinear optical properties of L-histidinium-L-tartrate hemihydrate," Journal of Molecular Structure, vol. 656, no. 13, pp. 265-273, 2003.

[11] H. Ratajczak, J. Barycki, A. Pietraszko, et al., "Preparation and structural study of a novel nonlinear molecular material: the L-histidinum dihydrogenarsenate orthoarsenic acid crystal," Journal of Molecular Structure, vol. 526, no. 1-3, pp. 269-278, 2000.

[12] S. Gokul Raj, G. Ramesh Kumar, R. Mohan, R. Jayavel, and B. Varghese, "Crystal structure and vibrational analysis of novel nonlinear optical L-histidinium tetrafluoroborate (L-HFB) single crystals," Physica Status Solidi B, vol. 244, no. 2, pp. 558-568, 2007.

[13] S. Gokul Raj, G. Ramesh Kumar, R. Mohan, B. Varghese, and R. Jayavel, "Crystal structure of single crystals of nonlinear optical L-histidinium trichloroacetate," Journal of Molecular Structure, vol. 825, no. 1-3, pp. 158-164, 2006.

[14] M. K. Marchewka, S. Debrus, and H. Ratajczak, "Vibrational spectra and second harmonic generation in molecular complexes of L-lysine with L-tartaric, D,L-malic, acetic, arsenous, and fumaric acids," Crystal Growth and Design, vol. 3, no. 4, pp. 587-592, 2003.

[15] A. M. Petrosyan, R. P. Sukiasyan, S. S. Terzyan, and V. M. Burbelo, "Interaction of lysine with iodic acid," Acta Crystallographica Section B, vol. 55, no. 2, pp. 221-225, 1999.

[16] R. Thenneti, K. Suriya Kumar, G. Ramesh Kumar, S. Gokul Raj, and R. Mohan, "L-lysinium trichloroacetate," Acta Crystallographica E, vol. 63, pp. o1706-o1707, 2007.

[17] H. Ratajczak, in Proceedings of the 3rd International Conference on Vibrational Spectroscopy in Materials Science, p. 21, Krakow, Poland, September 2000, Book of Abstracts.

[18] A. M. Petrosyan, R. P. Sukiasyan, H. A. Karapetyan, M. Y. Antipin, and R. A. Apreyan, "L-arginine oxalates," Journal of Crystal Growth, vol. 275, no. 1-2, pp. e1927-e1933, 2005. 
[19] S. Debrus, M. K. Marchewka, J. Baran, et al., "L-lysineL-tartaric acid: new molecular complex with nonlinear optical properties. Structure, vibrational spectra and phase transitions," Journal of Solid State Chemistry, vol. 178, no. 9, pp. 2880-2896, 2005.

[20] H. A. Petrosyan, H. A. Karapetyan, M. Y. Antipin, and A. M. Petrosyan, "Nonlinear optical crystals of L-histidine salts," Journal of Crystal Growth, vol. 275, no. 1-2, pp. e1919-e1925, 2005.

[21] D. T. J. Hurle, Handbook of Crystal Growth, vol. 2, NorthHolland, Amsterdam, The Netherlands, 1994.

[22] H. A. Meirs and F. Issac, Proceedings of the Royal Society of London, vol. A79, pp. 322-351, 1907.

[23] V. Bisker-Leib and M. F. Doherty, "Modeling crystal shape of polar organic materials: applications to amino acids," Crystal Growth and Design, vol. 3, no. 2, pp. 221-237, 2003.

[24] I. Weissbuch, R. Popovitz-Biro, M. Lahav, L. Leiserowitz, and Rehovot, "Understanding and control of nucleation, growth, habit, dissolution and structure of two- and threedimensional crystals using 'tailor-made' auxiliaries," Acta Crystallographica B, vol. 51, pp. 115-148, 1995.

[25] S. K. Kurtz and T. T. Perry, "A powder technique for the evaluation of nonlinear optical materials," Journal of Applied Physics, vol. 39, no. 8, pp. 3798-3813, 1968.

[26] D. P. Shoemaker, J. Donohue, V. Schomaker, and R. B. Corey, "The crystal structure of Ls-threonine," Journal of the American Chemical Society, vol. 72, no. 6, pp. 2328-2349, 1950.

[27] J. Janczak, D. Zobel, and P. Luger, "L-threonine at 12 K," Acta Crystallographica C, vol. 53, no. 12, pp. 1901-1904, 1997.

[28] J. J. Rodrigues Jr., L. Misoguti, F. D. Nunes, C. R. Mendonca, and S. C. Zilio, "Optical properties of L-threonine crystals," Optical Materials, vol. 22, no. 3, pp. 235-240, 2003.

[29] M. Lahav and L. Leiserowitz, "A stereochemical approach that demonstrates the effect of solvent on the growth of polar crystals: a perspective," Crystal Growth and Design, vol. 6, no. 3, pp. 619-624, 2006.

[30] Y. Tanaka and M. Matsuoka, "Selection of solvents for organic crystal growth from solution," Journal of Crystal Growth, vol. 99, no. 1-4, pp. 1130-1133, 1990.

[31] A. E. Nielsen and O. Söhnel, "Interfacial tensions electrolyte crystal-aqueous solution, from nucleation data," Journal of Crystal Growth, vol. 11, no. 3, pp. 233-242, 1971.

[32] L. K. Cheng, J. D. Bierlein, and A. A. Ballman, "Crystal growth of $\mathrm{KTiOPO}_{4}$ isomorphs from tungstate and molybdate fluxes," Journal of Crystal Growth, vol. 110, no. 4, pp. 697-703, 1991.

[33] K. Sangwal, "On the estimation of surface entropy factor, interfacial tension, dissolution enthalpy and metastable zone-width for substances crystallizing from solution," Journal of Crystal Growth, vol. 97, no. 2, pp. 393-405, 1989.

[34] P. Bennema and O. Söhnel, "Interfacial surface tension for crystallization and precipitation from aqueous solutions," Journal of Crystal Growth, vol. 102, no. 3, pp. 547-556, 1990.

[35] O. Söhnel, "Electrolyte crystal-aqueous solution interfacial tensions from crystallization data," Journal of Crystal Growth, vol. 57, no. 1, pp. 101-108, 1982.

[36] J. Nyvlt, R. Rychly, J. Gottfried, and J. Wurzelova, "Metastable zone-width of some aqueous solutions," Journal of Crystal Growth, vol. 6, no. 2, pp. 151-162, 1970.

[37] N. P. Zaitseva, L. N. Rashkovich, and S. V. Bogatyreva, "Stability of $\mathrm{KH}_{2} \mathrm{PO}_{4}$ and $\mathrm{K}(\mathrm{H}, \mathrm{D})_{2} \mathrm{PO}_{4}$ solutions at fast crystal growth rates," Journal of Crystal Growth, vol. 148, no. 3, pp. 276-282, 1995.

[38] R. Mohan Kumar, N. Gopalakrishnan, R. Jayavel, and P. Ramasamy, "Investigations on the nucleation kinetics of L-arginine phosphate single crystals," Crystal Research and Technology, vol. 34, no. 10, pp. 1265-1268, 1999.

[39] F. Joseph Kumar, D. Jayaraman, C. Subramanian, and P. Ramasamy, "Nucleation kinetic study of $\mathrm{KTiOPO}_{4}$ crystallizing from high temperature solution," Journal of Crystal Growth, vol. 137, no. 3-4, pp. 535-537, 1994.

[40] C. Razzetti, M. Ardoino, L. Zanotti, M. Zha, and C. Paorici, "Solution growth and characterization of L-alanine single crystals," Crystal Research and Technology, vol. 37, pp. 456465, 2002.

[41] Robert D. Simoni, Robert L. Hill, and Martha Vaughan, "The Synthesis and Structure of Threonine: Herbert E. Carter," The Journal of Biological Chemistry, vol. 112, pp. 769-773, 1935.

[42] S. Natarajan, H. Alex Devadoss, and K. Alwan, "Gel growth of single crystals of -glycine, L-threonine and N-glycylglycine," Crystal Research and Technology, vol. 23, no. 10-11, pp. 13431346, 2006.

[43] K. Sangwal and E. Olezyk, "Effect of $\mathrm{pH}$ on the electrical conductance of saturated aqueous solutions of $\mathrm{KH}_{2} \mathrm{PO}_{4}$ and its solubility," Crystal Research and Technology, vol. 25, pp. 65-70, 1990.

[44] N. Bloembergen, Non Linear Optics, Benjamin, New York, NY, USA, 1965.

[45] C. G. B. Garrett, "Nonlinear optics, anharmonic oscillators, and pyroelectricity," IEEE Journal of Quantum Electronics, vol. 4, pp. 70-84, 1968.

[46] W. Jamroz and J. Karniewicz, "The electro-optic kerr effect in noncentrosymmetric $\mathrm{KH}_{2} \mathrm{PO}_{4}$ and $\mathrm{KD}_{2} \mathrm{PO}_{4}$ monocrystals," Optical and Quantum Electronics, vol. 11, no. 1, pp. 23-27, 1979.

[47] S. K. Kurtz and F. N. H. Robinson, "A physical model of the electro-optic effect," Applied Physics Letters, vol. 10, no. 2, pp. 62-65, 1967.

[48] D. P. Akitt, C. J. Johnson, and P. D. Coleman, "Nonlinear susceptibility of CdTe," IEEE Journal of Quantum Electronics, vol. 6, no. 8, pp. 496-499, 1970.

[49] T. P. Sharma, S. Singh, and V. P. Singh, "Nonlinear susceptibility of zinc telluride," Journal of Applied Physics, vol. 47, no. 10, p. 4404, 1976.

[50] M. Sugie and K. Tada, "Generalized theory of nonlinear susceptibilities and linear electrooptic coefficients based on a three-dimensional anharmonic oscillator model," Japanese Journal of Applied Physics, vol. 12, pp. 215-225, 1973.

[51] N. Ramesh and K. Srinivasan, "Model of third-order susceptibilities for the Kerr effect in wide-bandgap crystals: optical science and engineering in India," Optical Engineering, vol. 33, pp. 1934-1936, 1994.

[52] G. Ramesh Kumar, S. Gokul Raj, K. A. Bogle, S. D. Dhole, V. N. Bhoraskar, and R. Mohan, "Investigations on the optical, thermal and surface modifications of electron irradiated Lthreonine single crystals," Applied Surface Science, vol. 254, no. 16, pp. 5231-5235, 2008.

[53] G. K. Mehta and A. P. Patro, "15 UD pelletron of the nuclear science centre-status report," Nuclear Instruments and Methods in Physics Research A, vol. 268, no. 2-3, pp. 334336, 1988.

[54] G. Ramesh Kumar, S. Gokul Raj, R. Sankar, R. Mohan, S. Pandi, and R. Jayavel, "Growth, structural, optical and 
thermal studies of non-linear optical L-threonine single crystals," Journal of Crystal Growth, vol. 267, no. 1-2, pp. 213 217, 2004.

[55] B. L. Silva, P. T. C. Freire, F. E. A. Melo, et al., "Polarized Raman spectra and infrared analysis of vibrational modes in L-threonine crystals," Brazilian Journal of Physics, vol. 28, no. 1, pp. 19-24, 1998.

[56] A. Pawlukojç, J. Leciejewicz, J. Tomkinson, and S. F. Parker, "Neutron scattering, infra red, Raman spectroscopy and ab initio study of L-threonine," Spectrochimica Acta A, vol. 57, no. 12, pp. 2513-2523, 2001.

[57] M. Barthes, H. N. Bordallo, F. Denoyer, et al., "Microtransitions or breathers in L-alanine?" European Physical Journal B, vol. 37, no. 3, pp. 375-382, 2004.

[58] G. Ramesh Kumar, S. Gokul Raj, T. Raghavalu, et al., "Effect of pH, thermal, electrical and thermomechanical properties of nonlinear optical L-threonine single crystals," Spectrochimica Acta A, vol. 68, no. 2, pp. 300-304, 2007.

[59] J. F. Nye, Physical Properties of Crystals, Clarendon Press, Oxford, UK, 1957.

[60] L. Misoguti, A. T. Varela, F. D. Nunes, et al., "Optical properties of L-alanine organic crystals," Optical Materials, vol. 6, no. 3, pp. 147-152, 1996.

[61] M. Ramanadham, S. K. Sikka, and R. Chidambaram, "Structure determination of L-theonine by neutron diffraction," Pramana, vol. 6, pp. 247-259, 1973.

[62] G. Dhanaraj, M. R. Srinivasan, H. L. Bhat, H. S. Jayanna, and S. V. Subramanyam, "Thermal and electrical properties of the novel organic nonlinear crystal L-arginine phosphate monohydrate," Journal of Applied Physics, vol. 72, no. 8, pp. 3464-3467, 1992.

[63] Reena Ittyachan, Preema C. Thomas, D. Prem Anand, M. Palanichamy, and P. Sagayaraj, "Growth and characterization of semiorganic non-linear optical LHB single crystal," Materials Chemistry and Physics, vol. 93, no. 2-3, pp. 272-276, 2005.

[64] G. Ramesh Kumar, S. Gokul Raj, R. Mohan, and R. Jayavel, "Growth, structural and spectral analyses of nonlinear optical L-threonine single crystals," Journal of Crystal Growth, vol. 275, no. 1-2, pp. e1947-e1951, 2005.

[65] G. Ramesh Kumar, S. Gokul Raj, R. Mohan, and R. Jayavel, "Influence of isoelectric $\mathrm{pH}$ on the growth linear and nonlinear optical and dielectric properties of L-threonine single crystals," Crystal Growth and Design, vol. 6, no. 6, pp. 1308-1310, 2006.

[66] B. W. Batterman and H. Cole, "Dynamical diffraction of Xrays by perfect crystals," Reviews of Modern Physics, vol. 36, no. 3, pp. 681-717, 1964.

[67] L. Krishan and G. Bhagavannarayana, "High-resolution diffuse X-ray scattering study of defects in dislocationfree silicon crystals grown by the float-zone method and comparison with Czochralski-grown crystals," Journal of Applied Crystallography, vol. 22, pp. 209-215, 1989.

[68] G. Bhagavannarayana, R. V. Ananthamurthy, G. C. Budakoti, B. Kumar, and K. S. Bartwal, "A study of the effect of annealing on Fe-doped $\mathrm{LiNbO}_{3}$ by HRXRD, XRT and FTIR," Journal of Applied Crystallography, vol. 38, no. 5, pp. 768771, 2005.

[69] N. Vijayan, S. Rajasekaran, G. Bhagavannarayana, et al., "Growth and characterization of nonlinear optical amino acid single crystal: L-alanine," Crystal Growth and Design, vol. 6, no. 11, pp. 2441-2445, 2006.

[70] G. Ramesh Kumar, S. Gokul Raj, T. Raghavalu, et al., "Crystallization kinetics and high-resolution X-ray diffraction analysis on nonlinear optical L-threonine single crystals," Materials Letters, vol. 61, no. 27, pp. 4932-4936, 2007.

[71] D. N. Nokogosyan, A Hand Book on Properties of Optical and Laser Related Materials, John Wiley \& Sons, New York, NY, USA, 1998.

[72] U. B. Ramabadran, A. L. Mcpherson, and D. E. Zelman, "Optical properties of deuterated zinc tristhiourea sulphate," Journal of Applied Physics, vol. 76, pp. 1150-1154, 1994.

[73] D. Eimerl, S. Velsko, L. Davis, F. Wang, G. Loiacono, and G. Kennedy, "Deuterated L-arginine phosphate: a new efficient nonlinear crystal," IEEE Journal of Quantum Electronics, vol. 25, no. 2, pp. 179-193, 1989.

[74] A. Yokotani, T. Sasaki, K. Yoshida, and S. Nakai, "Extremely high damage threshold of a new nonlinear crystal L-arginine phosphate and its deuterium compound," Applied Physics Letters, vol. 55, no. 26, pp. 2692-2693, 1989.

[75] R. S. Krishnan, K. Sankaranarayanan, and K. Krishnan, "Raman and infrared spectra of amino acids," Journal of the Indian Institute of Science, vol. 55, pp. 66-116, 1973.

[76] G. Ramesh Kumar, S. Gokul Raj, A. Saxena, A. K. Karnal, T. Raghavalu, and R. Mohan, "Deuteration effects on structural, thermal, linear and nonlinear properties of L-threonine single crystals," Materials Chemistry and Physics, vol. 108, no. 2-3, pp. 359-363, 2008.

[77] A. Pawlukoj, J. Leciejewicz, J. Tomkinson, and S. F. Parker, "Neutron scattering, infra red, Raman spectroscopy and ab initio study of L-threonine," Spectrochimica Acta A, vol. 57, no. 12, pp. 2513-2523, 2001.

[78] I. P. Kaminow, "Microwave dielectric properties of $\mathrm{NH}_{4} \mathrm{H}_{2} \mathrm{PO}_{4}$, and partially deuterated $\mathrm{KH}_{2} \mathrm{PO}_{4}$," Physical Review A, vol. 138, pp. 1539-1543, 1965.

[79] J. H. Ott and T. R. Sliker, "Linear electrooptic effects in $\mathrm{KH}_{2} \mathrm{PO}_{4}$ and its isomorphs," Journal of the Optical Society of America, vol. 54, pp. 1442-1444, 1964.

[80] R. S. Adhav, "Some physical properties of single crystals of normal and deuterated potassium dihydrogen arsenate. II: electro-optic and dielectric properties," Journal of Applied Physics, vol. 39, no. 9, pp. 4095-4098, 1968.

[81] C. Castiglioni, M. Del Zoppo, and G. Zerbi, "Molecular first hyperpolarizability of push-pull polyenes: relationship between electronic and vibrational contribution by a twostate model," Physical Review B, vol. 53, no. 20, pp. 1331913325, 1996.

[82] S. Singh, W. A. Bonner, T. Kyle, J. R. Potopowicz, and L. G. Van Uitert, "Second harmonic generation in d-threonine," Optics Communications, vol. 5, no. 2, pp. 131-133, 1972.

[83] R. W. Boyd, Non-Linear Optics, Academic Press, New York, NY, USA, 2nd edition, 2003.

[84] D. A. Kleinman, "Nonlinear dielectric polarization in optical media," Physical Review, vol. 126, no. 6, pp. 1977-1979, 1962.

[85] Z. Lin, Z. Wang, C. Chen, and M.-H. Lee, "Mechanism of linear and nonlinear optical effects of KDP and urea crystals," Journal of Chemical Physics, vol. 118, no. 5, pp. 2349-2356, 2003.

[86] G. Ramesh Kumar, S. Gokul Raj, V. Mathivanan, M. Kovendhan, T. Raghavalu, and R. Mohan, "Evaluation of NLO susceptibilities for L-threonine amino acid single crystals using anharmonic oscillator model," Optical Materials, vol. 30, no. 9, pp. 1405-1409, 2008.

[87] N. Kejalakshmy and K. Srinivasan, "Electro-optic properties of potassium hydrogen phthalate crystal and its application as modulators," Journal of Physics D, vol. 36, no. 15, pp. 17781782, 2003. 
[88] S. R. Marder, J. E. Sohn, and G. D. Stucky, "Materials for nonlinear optics-chemical perspectives," in Proceedings of the 199th National Meeting of the American Chemical Society ACS Symposium, p. 23, April 1990.

[89] R. C. Miller, "Optical second harmonic generation in piezoelectric crystals," Applied Physics Letters, vol. 5, no. 1, pp. 17$19,1964$.

[90] C. G. B. Garrett and F. N. H. Robinson, "Miller's phenomenological rule for computing nonlinear susceptibilities," IEEE Journal of Quantum Electronics, vol. 2, pp. 328329, 1966.

[91] S. Ebraheem, W. B. Beshir, S. Eid, R. Sobhy, and A. Kovacs, "Spectrophotometric readout for an alanine dosimeter for food irradiation applications," Radiation Physics and Chemistry, vol. 67, no. 3-4, pp. 569-573, 2003.

[92] K. Van Laere, J. Buysse, and P. Berkvens, "Alanine in highdose dosimetry: spectrophotometric and electrochemical readout procedures compared with ESR," Applied Radiation and Isotopes, vol. 40, no. 10-12, pp. 885-895, 1989.

[93] B. Ciesielski and L. Wielopolski, "The effects of dose and radiation quality on the shape and power saturation of the EPR signal in alanine," Radiation Research, vol. 140, no. 1, pp. 105-111, 1994.

[94] J. P. Adloff, Ed., Handbook of Hot Atom Chemistry, Kodansha, Tokyo, Japan, 1992.

[95] J. Butler, B. M. Hoey, and A. J. Swallow, "Radiation chemistry," Annual Reports Section C, vol. 86, pp. 49-93, 1989.

[96] S. J. Pachuta and R. G. Cooks, "Mechanisms in molecular SIMS," Chemical Reviews, vol. 87, no. 3, pp. 647-669, 1987.

[97] D. S. Billington and J. J. Cawford, Radiation Damage in Solids, Princeton University Press, Princeton, NJ, USA, 1961.

[98] P. G. Fuochi, "Irradiation of power semiconductor devices by high energy electrons: the Italian experience," Radiation Physics and Chemistry, vol. 44, no. 4, pp. 431-440, 1994.

[99] R. P. Agarwal, "Irradiation induced physical property changes in materials," in Proceedings of the Symposium on Radiation Effects in Solids, vol. 2, November 1983.

[100] S. Ishwar Bhat, N. Govinda Nayak, V. Rao, V. Ganesan, H. S. Nagaraj, and D. K. Avasthi, "AFM studies of swift heavy ion and electron irradiated mixed barium strontium borate nonlinear optical crystal," Radiation Measurements, vol. 36, no. 1-6, pp. 695-698, 2003.

[101] S. Ishwar Bhat, P. Mohan Rao, A. P. Ganesh Bhat, and D. K. Avasthi, "Irradiation effects on the optical properties of a new NLO mixed borate crystal," Surface and Coatings Technology, vol. 158-159, pp. 725-728, 2002.

[102] ESTAR, "Stopping range and tables for electrons," 2003, http://physics.nist.gov/PhysRefData/Star/Text/ESTAR.html .

[103] E. Winkler, P. Etchegoin, A. Fainstein, and C. Fainstein, "Luminescence and resonant Raman scattering of color centers in irradiated crystalline L-alanine," Physical Review B, vol. 57, no. 21, pp. 13477-13484, 1998.

[104] J. Kopniczky, C. T. Reimann, A. Hedin, B. U. R. Sundqvist, P. Tengvall, and R. Erlandsson, "Scanning-force-microscopy study of MeV-atomic-ion-induced surface tracks in organic crystals," Physical Review B, vol. 49, no. 1, pp. 625-628, 1994.

[105] J. F. Nye, Physical Properties of Crystals, Clarendon Press, Oxford, UK, 1957.

[106] R. E. Johnson, B. U. R. Sundqvist, A. Hedin, and D. Fenyo, "Sputtering by fast ions based on a sum of impulses," Physical Review B, vol. 40, no. 1, pp. 49-53, 1989.

[107] D. Fenyo, B. U. R. Sundqvist, B. R. Karlsson, and R. E. Johnson, "Molecular-dynamics study of electronic sputtering of large organic molecules," Physical Review B, vol. 42, no. 4, pp. 1895-1902, 1990.

[108] S. Ebraheem, W. B. Beshir, S. Eid, R. Sobhy, and A. Kovacs, "Spectrophotometric readout for an alanine dosimeter for food irradiation applications," Radiation Physics and Chemistry, vol. 67, no. 3-4, pp. 569-573, 2003.

[109] R. Mishra, S. P. Tripathy, K. K. Dwivedi, et al., "Effect of electron irradiation on polytetrafluoro ethylene," Radiation Measurements, vol. 37, no. 3, pp. 247-251, 2003.

[110] R. Vijayalakshmi Rao, P. Mohan Rao, and M. H. Shridhar, "Effect of electron irradiation on P4VP/PTSA complex and $\mathrm{P} 4 \mathrm{VP} / \mathrm{Phth}$ locyanine composites," Nuclear Instruments and Methods in Physics Research B, vol. 187, no. 3, pp. 331-339, 2002.

[111] G. Ramesh Kumar, S. Gokul Raj, K. A. Bogle, S. D. Dhole, V. N. Bhoraskar, and R. Mohan, "Spectral, electrical and thermal properties of electron-irradiated L-threonine single crystals," Radiation Effects and Defects in Solids, vol. 163, no. 3, pp. 223-228, 2008.

[112] J. R. Bird and J. S. Williams, Ion Beam for Materials Analysis, Academic Press, Sydney, Australia, 1989.

[113] S. O. Kucheyev and T. E. Felter, "Structural disorder produced in $\mathrm{KH}_{2} \mathrm{PO}_{4}$ by light-ion bombardment," Journal of Applied Physics, vol. 95, no. 12, pp. 8475-8477, 2004.

[114] T. Som, M. S. Navati, and V. N. Kulkarni, "Physico-chemical changes in ion-irradiated KDP," Nuclear Instruments and Methods in Physics Research B, vol. 179, no. 4, pp. 551-556, 2001.

[115] T. Som, S. Dhar, S. N. Minwalla, and V. N. Kulkarni, "Hydrogen depletion from $\mathrm{KH}_{2} \mathrm{PO}_{4}$ under $\mathrm{He}^{+}$ion bombardment," Nuclear Instruments and Methods in Physics Research B, vol. 122, no. 2, pp. 244-246, 1997.

[116] K. Suriya Kumar, S. Gokul Raj, G. Ramesh Kumar, and R. Mohan, "50 MeV Li3+ Irradiation Effects on Structural, Optical and Surface Properties of L-Alanine Single Crystals," Science of Advanced Materials, vol. 1, pp. 286-291, 2009.

[117] P. Sreeramana Aithal, H. S. Nagaraja, P. Mohan Rao, D. K. Avasthi, and A. Sarma, "Effect of high energy ion irradiation on electrical and optical properties of organic nonlinear optical crystals," Vacuum, vol. 48, no. 12, pp. 991-994, 1997.

[118] P. Sreeramana Aithal, H. S. Nagaraja, P. Mohan Rao, D. K. Avasthi, and A. Sarma, "Effect of high energy ion irradiation on electrical and optical properties of parahydroxy acetophenone," Journal of Applied Physics, vol. 81, no. 11, pp. 7526-7528, 1997.

[119] A. M. Foti, F. Milano, and L. Torrisi, "Amino acid decomposition induced by $\mathrm{keV}$ ion irradiation," Nuclear Instruments and Methods in Physics Research B, vol. 46, no. 1-4, pp. 361363, 1990.

[120] W. Huang, Z. Yu, and Y. Zhang, "Repair by radiation itself or not? A study of threonine irradiated by keV ion," Nuclear Instruments and Methods in Physics Research B, vol. 134, no. 2, pp. 202-208, 1998.

[121] H. Matzke, "Radiation damage in crystalline insulators, oxides and ceramic nuclear fuels," Radiation Effects and Defects in Solids, vol. 64, no. 1-4, pp. 3-33, 1983.

[122] M. Toulemonde, S. Bouffard, and F. Studer, "Swift heavy ions in insulating and conducting oxides: tracks and physical properties," Nuclear Instruments and Methods in Physics Research B, vol. 91, no. 1-4, pp. 108-123, 1994.

[123] Z. G. Wang, C. Dufour, E. Paumier, and M. Toulemonde, "The Se sensitivity of metals under swift-heavy-ion irradiation: a transient thermal process," Journal of Physics: Condensed Matter, vol. 6, no. 34, pp. 6733-6750, 1994. 
[124] R. L. Fleischer, P. B. Price, and R. M. Walker, Nuclear Tracks in Solids: Principles and Applications, University of California Press, Berkely, Calif, USA, 1975.

[125] J. F. Ziegler, J. D. Biersack, and U. Littmark, The Stopping and Ranges of Ions in Solids, Pergamon, New York, NY, USA, 1988.

[126] P. Zanola, P. Colombi, E. Bontempi, R. Roberti, M. Gelfi, and L. E. Depero, "Glancing-incidence X-ray diffraction for depth profiling of polycrystalline layers," Journal of Applied Crystallography, vol. 39, no. 2, pp. 176-179, 2006.

[127] N. Rozlosnik, L. Sajo Bohus, C. Birattari, E. Gadioli, L. P. Biro, and K. Havancsak, "Direct observation of latent nuclear tracks in organic material by atomic force microscopy," Nanotechnology, vol. 8, no. 1, pp. 32-34, 1997.

[128] D. Fenyo and R. E. Johnson, "Computer experiments on molecular ejection from an amorphous solid: comparison to an analytic continuum mechanical model," Physical Review B, vol. 46, no. 9, pp. 5090-5099, 1992.

[129] J. Kopniczky, A. Hallen, N. Keskitalo, C. T. Reimann, and B. U. R. Sundqvist, "MeV-ion-induced defects in organic crystals," Radiation Measurements, vol. 25, no. 1-4, pp. 4750, 1995.

[130] G. Ramesh Kumar, S. Gokul Raj, V. Mathivanan, et al., "Swift ion irradiation effects on L-threonine amino acid single crystals," Journal of Physics Condensed Matter, vol. 19, no. 46, Article ID 466108, 2007. 

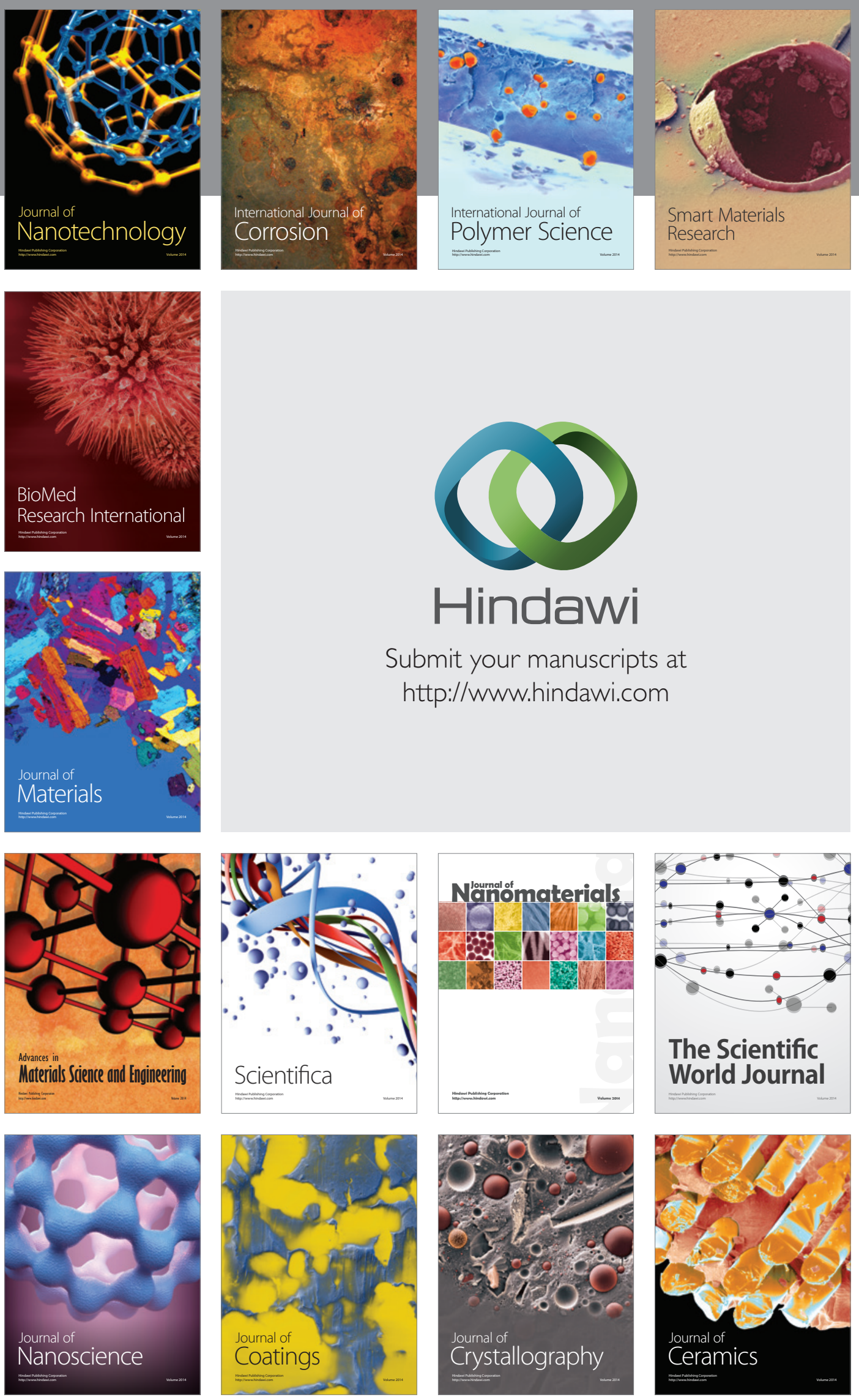

The Scientific World Journal

Submit your manuscripts at

http://www.hindawi.com

\section{World Journal}

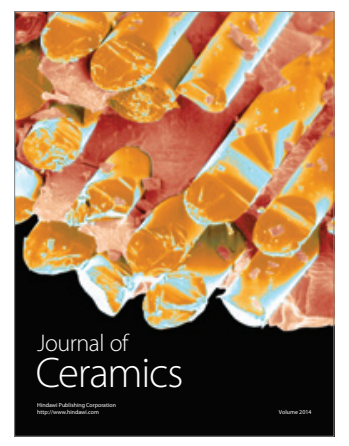

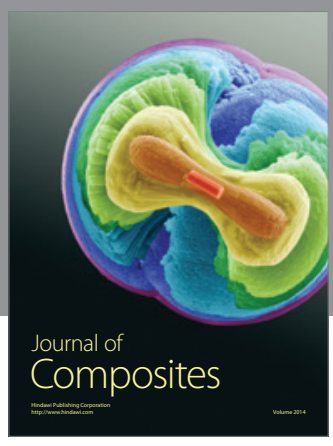
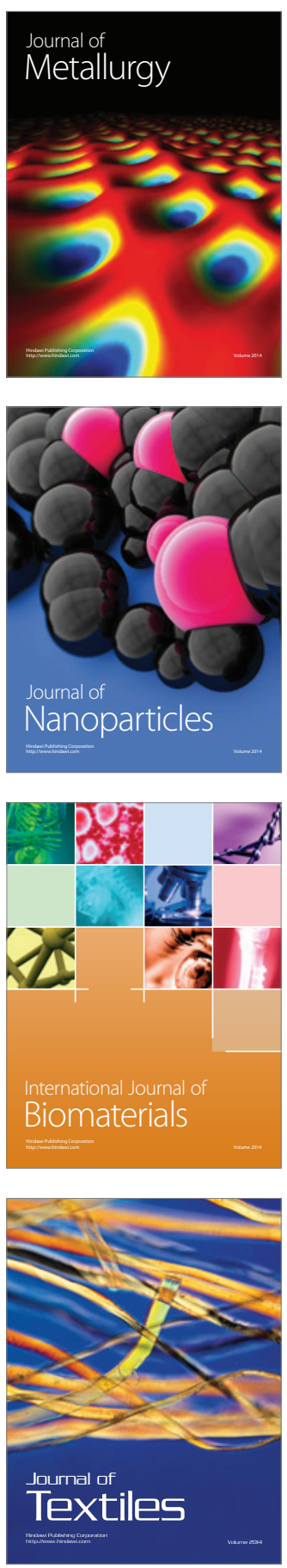